\title{
High Energy Physics Advisory Panel's Subpanel on Vision for the Future of High-Energy Physics
}

\section{May 1994}

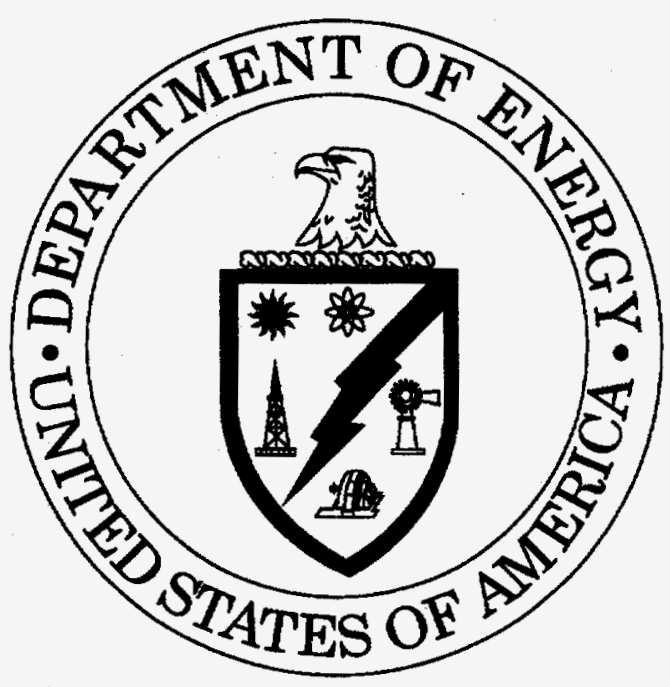

U.S. Department of Energy Office of Energy Research Division of High Energy Physics Washington, D.C. 20585 


\section{STANFORD UNIVERSITY \\ STANFORD, CALIFORNIA 94305-4060}

Secretary Hazel R. O'Leary

U.S. Department of Energy

Washington, D.C. 20585

Dear Madam Secretary:

I am happy to transmit to you the report of the "High Energy Physics Advisory Panel's Subpanel on Vision for the Future of High Energy Physics" chaired by Professor sidney D. Drell. This Subpanel was formed to address the issues posed in your letter to me dated November 4, 1993. The full Panel discussed the report at its May 23, 1994, meeting and enthusiastically and unanimously endorsed it.

On behalf of the whole United states High Energy Physics community we would like to thank Professor Drell and the members of the subpanel for the extremely conscientious effort that they have put into preparation of this report. We are also very impressed with the extent to which the community's views were solicited, discussed, and considered and believe that the report is representative both of the aspirations and of the concerns of the U.S. high energy physicists.

The report represents a carefully considered vision of how the United states can remain among the leaders in the field of particle physics even in these tight budgetary times. We sincerely hope that the Department of Energy and the other relevant agencies and branches of the U.S. Government endorse and implement the subpanel's recommendations. Our community is ready to work with you, your staff, and other Government bodies to achieve the vision that is so thoughtfully mapped out in this report.

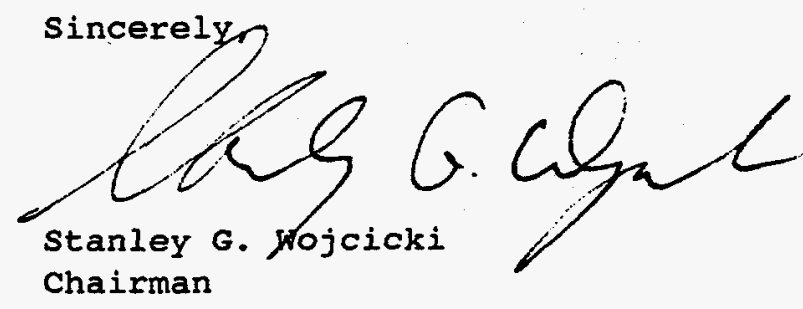

High Energy Physics Advisory Panel

cc:

Martha Krebs, DOE

Robert Eisenstein, NSF 
P. O. Box 4349

Stanford, CA 94309

(415) 926-2664

Telefax: (415) 926-4500

Bitnet Address:

BROSE at SLACVM

May 15, 1994

\section{Prof. Stanley Wojcicki}

Chairman

High Energy Physics Advisory Panel

Physics Department

Stanford University

Stanford, CA 94305

\section{Dear Stan:}

Herewith I submit to you the report of the 1994 HEPAP Subpanel on the "Future Vision" of the U.S. High Energy Physics Program. I have appended the charge for this study as stated by Secretary Hazel O'Leary in her letter to you dated November 4, 1993, together with other pertinent information chronicling the schedule and agenda of our meetings, and of town meetings organized by the Division of Particles and Fields of the American Physical Society. I also list the names of the many colleagues who wrote to the Subpanel with their views, concerns, and recommendations. Their letters were very valuable input to our deliberations.

I want to express deep appreciation to the Subpanel members who worked with great diligence, dedication, enthusiasm, and commitment to help accomplish the very difficult task that we faced. We also had excellent support and cooperation from the DOE officials and the staff of its Division of High Energy Physics. It is a special pleasure to acknowledge the superb and invaluable assistance of Kate Metropolis, our scientific editor in the preparation of this report. I also wish to acknowledge excellent paragraphs on the general quest for understanding from Professor James S. Trefl of George Mason University and Dr. Michael Riordan of SLAC that helped frame our presentation at the beginning of this report.

I hope HEPAP will endorse the vision and strategy for the future that we have recommended in this effort. I also hope that both the U.S. high-energy physics community and the U.S. government will endorse and implement its recommendations.

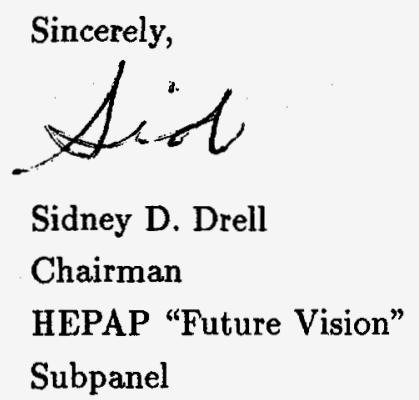

SDD:bgr

Enclosures 


\section{DISCLAIMER}

This report was prepared as an account of work sponsored by an agency of the United States Government. Neither the United States Government nor any agency thereof, nor any of their employees, make any warranty, express or implied, or assumes any legal liability or responsibility for the accuracy, completeness, or usefulness of any information, apparatus, product, or process disclosed, or represents that its use would not infringe privately owned rights. Reference herein to any specific commercial product, process, or service by trade name, trademark, manufacturer, or otherwise does not necessarily constitute or imply its endorsement, recommendation, or favoring by the United States Government or any agency thereof. The views and opinions of authors expressed herein do not necessarily state or reflect those of the United States Government or any agency thereof. 


\section{DISCLAIMER}

Portions of this document may be illegible in electronic image products. Images are produced from the best available original document. 


\section{CONTENTS}

\section{EXECUTIVE SUMMARY}

CONCLUSIONS and RECOMMENDATIONS

Chapter I. Why Take the Next Step? ............ 1

Chapter II. Elements of a World-Class Program in High-Energy Physics ................ 5

Chapter III. Elementary Particle Physics Today ......... 9

Chapter IV. The Existing Accelerator-Based Program $\ldots \ldots 23$

Chapter V. The Energy Frontier .................. 39

Chapter VI. Particle Physics without Accelerators ........ 55

Chapter VII. Our Vision of the Future $\ldots \ldots \ldots \ldots \ldots . \ldots 59$

Chapter VIII. Realizing the Vision $\ldots \ldots \ldots \ldots \ldots \ldots \ldots 63$

Chapter IX. The Importance of International Collaboration . . 73

Chapter X. Toward Improved Management of the Program . 79

Chapter XI. Using Superconducting Super Collider Assets . . 87

\section{Figures and Tables}

Figure 1. High Energy Physics Funding (1960-1995) ....... v

Figure 2. Budget Proposal for the High Energy Physics Program $(1995-2002) \ldots \ldots \ldots \ldots \ldots \ldots$. . . . . . . . . .

Figure 3. Same as 2, with suppressed zero $\ldots \ldots \ldots \ldots \ldots \ldots$

Table A. Elementary Particles and their Properties $\ldots \ldots \ldots 11$

Table B. Funding schedules for the Fermilab Main Injector and the SLAC B-factory ...............66 


\section{Appendices}

A. The Superconducting Super Collider Project: A Summary

B. Secretary O'Leary's letter charging the High Energy Physics Advisory Panel (HEPAP) to constitute the Subpanel

C. Membership of the HEPAP Subpanel on Vision for the Future of HighEnergy Physics

D. Subpanel Chairman Drell's letters (3) to the high-energy physics community

E. Subpanel Meeting Agendas

F. Town Meeting Agendas

G. Authors of letters to the Subpanel

H. International Committee on Future Accelerators (ICFA) Guidelines 


\section{EXECUTIVE SUMMARY CONCLUSIONS AND RECOMMENDATIONS}

The goal of particle physics is to understand the nature of matter at its deepest level, to answer the questions: What are the fundamental building blocks that make up the universe? What laws of nature determine their interactions at any time and place in the universe?

Twentieth century civilization has inherited a long and rich scientific tradition that began with the Greek philosophers. From the chemists of the eighteenth century who explored the behavior of atoms, to the physicists of the twentieth century who unlocked the secrets of the nucleus, scientists have probed nature in ever finer detail in search of its basic constituents and fundamental physical laws.

Each step along the way has given human beings a new way of looking at the universe, and each has, in time, led to new possibilities for applying basic knowledge for the benefit of humanity. The discovery of atoms, for example, turned alchemy into chemistry and gave us control of the elements. During the twentieth century, physicists went a step further and revealed that the chemical elements are assembled from still more elementary building blocks-the protons and neutrons that make up the nucleus, and the electrons that orbit around them. This work led to advances in medicine, electronics, computing and many other technologies. More recently, particle physicists have discovered that the particles in the nucleus are made up of even more fundamental constituents: the quarks. Who knows where this discovery will lead?

One thing is certain: over the years, many beautiful-and often surprising-experimental results have revealed a remarkable simplicity that underlies not only the world around us, but phenomena anywhere in the universe, at any time in its history. Indeed, as we have been able to study collisions between particles at higher and higher energies, we have begun to see patterns in their interactions that give us reason to hope that one day all interactions can be described by a single, unified theory.

The desire to understand the laws that govern the behavior of matter and energy, space and time, has inspired us to build particle accelerators and detectors that are marvels of scientific imagination and technological ingenuity. Over the past forty years, many important new discoveries have been made by experimental collaborations at high-energy particle accelerators. In addition, these accelerators have made it possible for human beings to experimentally study the early history of the cosmos. A combination of astrophysical and accelerator-based inquiries has enabled physicists to retrace the history of the 
universe back to less than a billionth of a second after the Big Bang. To understand the unimaginably hot and dense conditions that existed at that time, we must understand the physics of elementary particles. The synergy between astrophysics and particle physics enables us to hone our comprehension of our universe and our theories of the fundamental forces in nature. There is growing evidence for a deep connection between the physics of the infinitely small and the largest structures in the cosmos.

The pursuit of particle physics has opened the door to important new questions. Recently, for instance, experimenters at Fermilab announced evidence for the top quark, believed to be the last member of a family of six such particles. But, a priori, no one could predict what its mass would be. No one can explain why it weighs orders of magnitude more than its siblings. And no one has found a meaningful pattern in the masses of any of the quarks or elementary particles that have been discovered. Today, we are challenged to understand the origin of mass and other equally compelling issues: Why does there appear to be more matter than antimatter in the universe-a fact that ultimately enabled stars, planets, trees, and human beings to exist? And what makes up the $90 \%$ of the mass of the universe that we know exists but have not been able to see?

Answering these questions, and looking deeper and deeper into the heart of matter, requires experiments with particles of higher and higher energies. We must build on our work at today's cutting edge in moving on to tomorrow's frontiers. The high-energy frontier has historically provided the most direct path to new breakthroughs. This was the motivation for the Superconducting Super Collider (SSC), which the high-energy physics community expected to lead to the next big step in understanding. Throughout the past decade, the community endorsed the construction of the SSC as the highest priority in the U.S. particle physics program.

There are scientifically compelling reasons for exploring particle collisions at the energies the SSC would have reached. There was also a strong conviction that we could master the technological challenges to its successful construction and operation. Moreover, its design incorporated an important potential for future growth to higher energies and more intense beams, which promised a unique opportunity extending well into the twenty-first century, to address some of the most important questions about the nature of matter.

Cancellation of the SSC is a severe loss to worldwide high-energy physics. It is an especially severe loss to the American community, which now faces the challenge of creating a new vision for the future-one that continues to place scientific excellence at the core, minimizes the damage to the careers of young physicists from the loss of the SSC, maximizes the efficiency of research, and is acceptable and affordable to the American people. 
This subpanel was charged with developing and characterizing a strategy to realize that vision; a strategy that will continue to address the most important scientific questions, ensure the U.S. a position among the world's leaders in this field, and look to more effective international collaborations to realize our scientific goals. In addition, we were asked to suggest how best to use the assets and accomplishments of the SSC project.

\section{Conclusion 1}

We have inherited a great tradition of scientific inquiry. The field of particle physics has made dramatic progress in understanding the fundamental structure of matter. Recent discoveries and technological advances enable us to address such compelling scientific issues as the origin of mass, the underlying cause of the preponderance of matter over antimatter, and the nature of the invisible matter that accounts for up to $90 \%$ of the mass of the universe.

\section{Recommendation 1}

As befitting a great nation with a rich and successful history of leadership in science and technology, the United States should continue to be among the leaders in the worldwide pursuit of the answers to fundamental questions of particle physics.

\section{Status of the Field}

The current complement of U.S. high-energy accelerator laboratories is world class. It provides university students, faculty, and national laboratory physicists with access to experimental frontiers. These accelerators and detectors at the Fermi National Accelerator Laboratory (Fermilab), the Stanford Linear Accelerator Center (SLAC), Brookhaven National Laboratory (BNL), and Cornell are now addressing basic questions of particle physics and will continue to do so for some time. With the completion of scheduled upgrades in 1999-the Main Injector at Fermilab, the B-factory at SLAC, and the Cornell Electron Storage Ring-II at Cornell-the U.S. will maintain frontier capabilities, and the potential to make significant discoveries, during at least the next decade. In addition, there are significant U.S. particle physics research efforts that do not rely on accelerators. These domestic resources are complemented by unique opportunities abroad for U.S. scientists to conduct research, just as many foreign collaborators contribute substantially to the 
work in this country. With adequate support to upgrade and operate these accelerators and detectors and to respond to new experimental initiatives, the U.S. high-energy physics program will remain a world leader for a decade or more.

However, we must look beyond the horizon of this program if the U.S. is to remain productive, responsive, and a world leader in this field. To achieve this goal, two additional elements are essential:

- We must continue to collaborate in international scientific endeavors that are exploring or will explore new high-energy frontiers, whether in the United States or abroad.

- We must create opportunities at laboratories and at universities to open new research frontiers through a strong program of advanced accelerator and detector research and development. Technical advances generated by this program, together with advances in scientific understanding from ongoing experiments, will be important guides in making choices and setting priorities for the longer-term future.

The U.S. high-energy physics program is severely challenged as it faces FY1995. At the same time as its scientific potential has been growing, the program is being strained by the budget reductions that have occurred in recent years (see Figure 1). The loss in annual research support-that is, in the annual appropriation of operating and equipment funds-from FY1990 to the President's budget submission to Congress for FY1995 is about $\$ 135 \mathrm{M}$ in current dollars, when inflation is included. This represents about a $20 \%$ reduction in buying power.

With the termination of the SSC, additional funds were lost to the entire high- energy physics program, both from that project and from the Texas National Research Laboratory Commission (TNRLC). This loss has led to a scarcity of research opportunities and positions for young high-energy physicists. This situation is further aggravated because a number of new experimental research groups were formed throughout the nation in anticipation of the SSC. As a result, many careers are in jeopardy, and many young people believe that future prospects are bleak for continuing this historic scientific quest. 


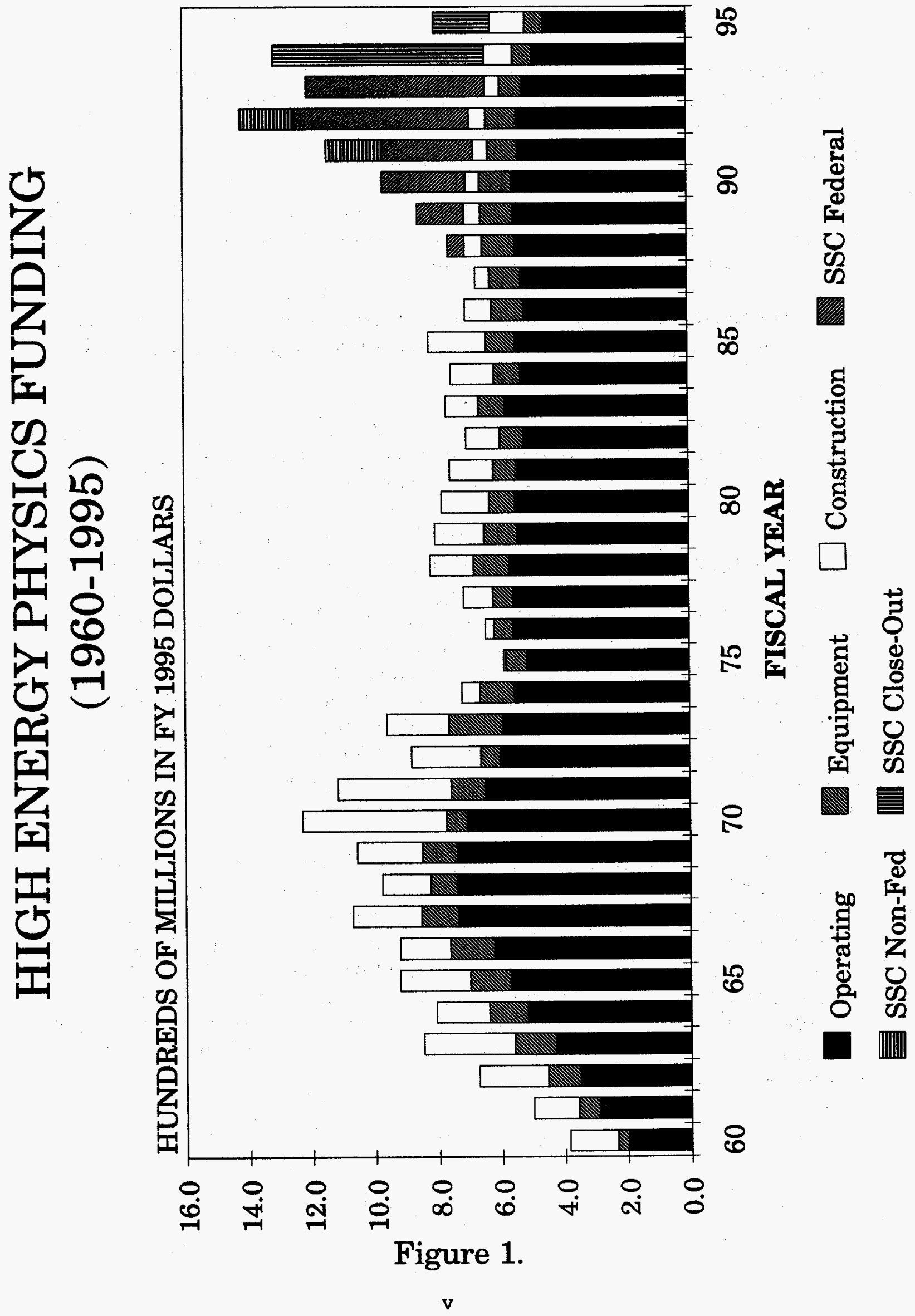




\section{Strategies and Opportunities for the Future}

The Tevatron at Fermilab is now the highest energy accelerator in the world. Ten years from now, the Large Hadron Collider (LHC) at the European Laboratory for Particle Physics (CERN) will offer a unique prospect for advancing to the highest energy frontiers. The LHC, planned to begin operation at CERN circa the year 2003, will be a high-performance protonproton collider with seven times the energy and up to 100 times the luminosity of the Tevatron. Research and development in the U.S. and in Europe have capitalized on rapid change in electronics technologies to create particle detectors that promise to meet the stringent demands of the LHC environment. The LHC will open new windows to discovery and present important opportunities to confront questions posed by current experiments and theories.

Besides providing U.S. physicists with access to new scientific territory, participation in the LHC would benefit the U.S. in other ways beyond the lifespan of the accelerator itself. Successful construction and operation of the Tevatron, as well as research and design for the SSC, have provided U.S. scientists and engineers with the world-class experience and knowledge needed for the design, implementation, and operation of large detectors and superconducting accelerators. This priceless human resource would enable the U.S. to make important contributions to the LHC; furthermore, sustaining this collective expertise over the next decade will be invaluable in keeping the U.S. at the forefront of proton accelerator technologies for the long-term future. In addition, helping to build the LHC, and developing strategies and mechanisms needed for global cooperation on large science projects, would further strengthen our credibility as a capable host for such projects, in all fields of science.

The LHC project is still in its design stage, so the time is appropriate for the U.S. to initiate participation. In June of this year, CERN will seek approval from its member states for the LHC project. CERN desires interregional collaboration in the design and construction of the accelerator and detectors.

While the LHC will be a great step on the energy frontier, it will not answer all the important questions. To ensure the long-term future of the field, a number of diverse approaches to advanced accelerator and detector research and development should be encouraged. The technology of the LHC does not exhaust the possibilities for proton storage rings. Preliminary examination indicates that it may become practical to build a proton collider with beams of up to ten times the energies of the LHC, using technology that could be developed during the next decade. For the U.S. to maintain its place among the leaders of the world high-energy physics community, it will be important to participate in regional or global collaborations to carry out the research and 
development required for such a future machine. Investigation also should be pursued of a possible expansion of the Tevatron/Main Injector complex at Fermilab as an intermediate step; possibilities may emerge that offer new scientific opportunities, thereby enhancing fruitful research in the U.S. at a hadron collider.

Our experience over the past four decades has provided ample evidence of the great importance of probing nature with both electron and proton collisions. Electron-positron colliders offer new and uniquely important experimental opportunities. An international consensus has been forged on the physics goals and parameters of a future linear collider. A worldwide effort is under way to develop the understanding of accelerator physics and the technology needed to build the next-generation electron-positron collider, beyond the currently operating Stanford Linear Collider (SLC). This international research and development program is focused on formulating conceptual designs, validating the technology, and generating reliable cost estimates in the second half of this decade. Prototype accelerators are now under construction. For the U.S. to maintain a leading role in the development of a future-generation linear collider, it is important to support research and development at U.S. laboratories and universities involved in this work.

For the longer term, it is important to investigate novel acceleration systems, as well as techniques for accelerating particles other than protons and electrons. Many intriguing ideas are being discussed, but much work remains to be done before it will be possible to determine which, if any, are practical for high-energy physics research.

\section{Conclusion 2}

To sustain excellence in the U.S. high-energy physics program for two decades and beyond, three elements are essential:

- a flexible, diverse, and dynamic ongoing research effort to address scientifically compelling questions. This implies strong support for university groups, effective use and timely upgrades of domestic accelerators, and an active program of nonaccelerator-based inquiries.

- vigorous studies to develop and master the technologies for future accelerators and detectors, and

- significant participation at the highest energy frontier, for which the best current opportunity beyond the Tevatron is through international collaboration on the LHC at CERN. 
When we consider what funding profiles are necessary to realize this vision, two important points emerge clearly. The first is that the greatest immediate need is to revitalize the current program to serve as a healthy and balanced base on which to build a future. This is the essential starting point for any successful strategy to realize our vision. The second is that an effective American presence on the high-energy frontier is essential for the long-term vitality of the U.S. program. The first requires a temporary budget increase over the next three years. The second can be fit in under a constant-level-ofeffort budget over the long term, without making a large immediate demand for funding support (see Figures 2 and 3). What is needed is an early "go" decision by the U.S. government to enable scientists to plan effectively for participating in the research and development toward construction of the accelerator and detectors at the LHC.

The build-up in sizable U.S. spending levels as part of the LHC collaboration can be phased in as the current commitments-to the construction of the Main Injector at Fermilab and the B-factory at SLAC, and to the operation of the Alternating Gradient Synchrotron (AGS) at BNL for highenergy physics-wind down. These reductions in budgetary obligations of the U.S. high-energy physics program were anticipated in planning for the start of physics research at the SSC. They now provide important flexibility to meet long-term needs, so that with modest budgetary commitments, the U.S. can realistically support a world-class high-energy physics program. An effective collaboration in the LHC will offset in part the lost research opportunities of the SSC and keep the U.S. high-energy physics program at the energy frontier into the twenty-first century.

\section{Conclusion 3}

A temporary and modest bump of $\$ 50 \mathrm{M} /$ year in the total funding for three years from FY1996 through FY1998, followed by a return to a constant-level-of-effort budget at the level of the President's proposed FY1995 budget, as shown in Figures 2 and 3, would revitalize the ongoing research program and sustain it through the construction years of the two upgrades at Fermilab and SLAC. Within that budget profile, it would be possible to reverse the FY1995 cut in the research (operations plus equipment) budget, permitting its restoration to the FY1994 level in buying power and, thus, the productive use of investments made to date; and also to initiate significant participation in building the LHC, with the level of commitment growing slowly until FY1997 and reaching its full level in FY1999. 


\section{Budget Proposal for the High Energy Physics Program (1995-2002)}

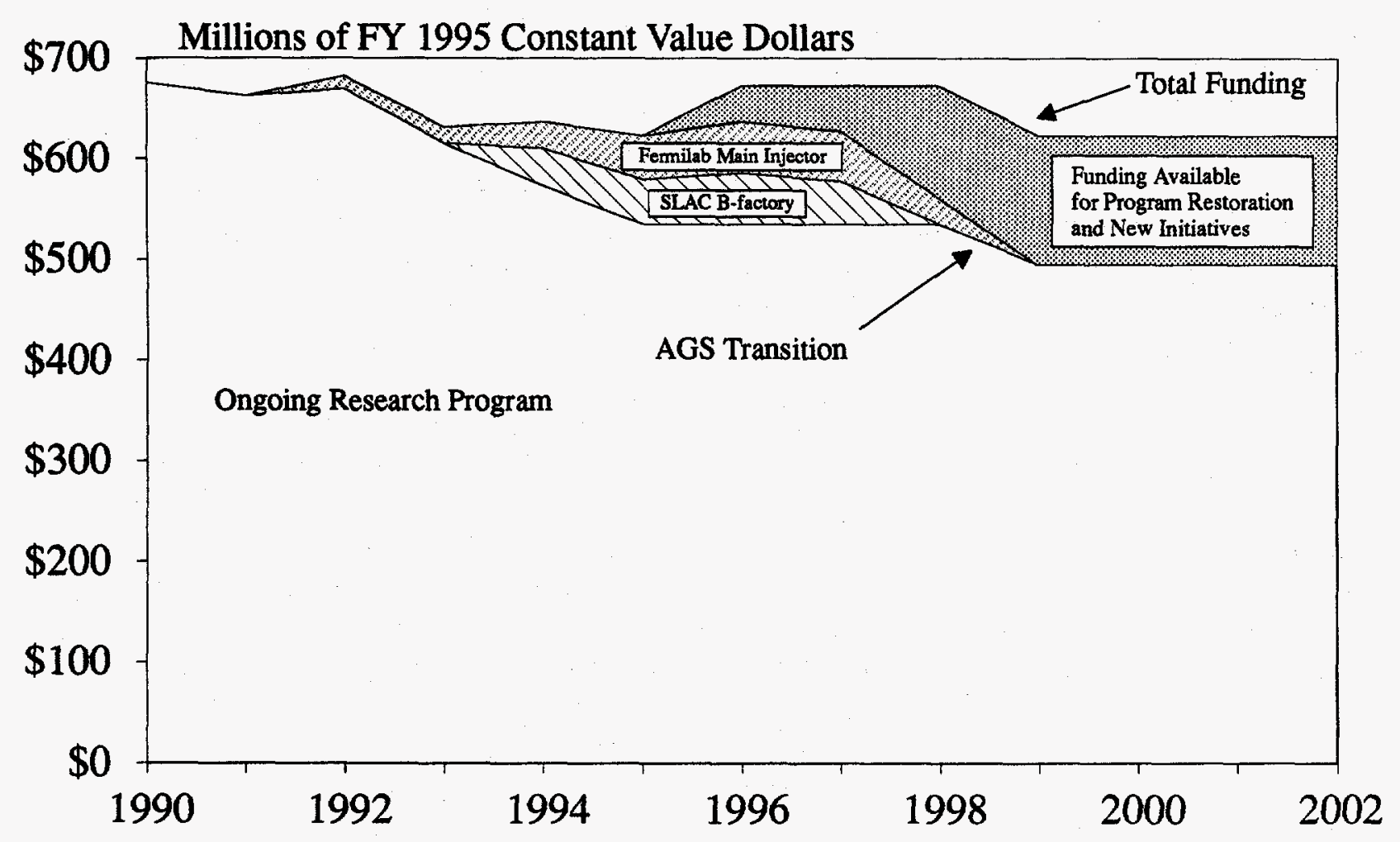

Fiscal Year

Figure 2. The total funding beyond FY1995 is set at the FY1995 level plus the proposed $\$ 50 \mathrm{M}$ three year bump. The Fermilab Main Injector and the SLAC B-factory are shown on the basis of their approved funding profiles. The funding above that shown for the construction projects is available for program restoration and new initiatives. 


\section{Budget Proposal for the High Energy Physics Program (1995-2002)}

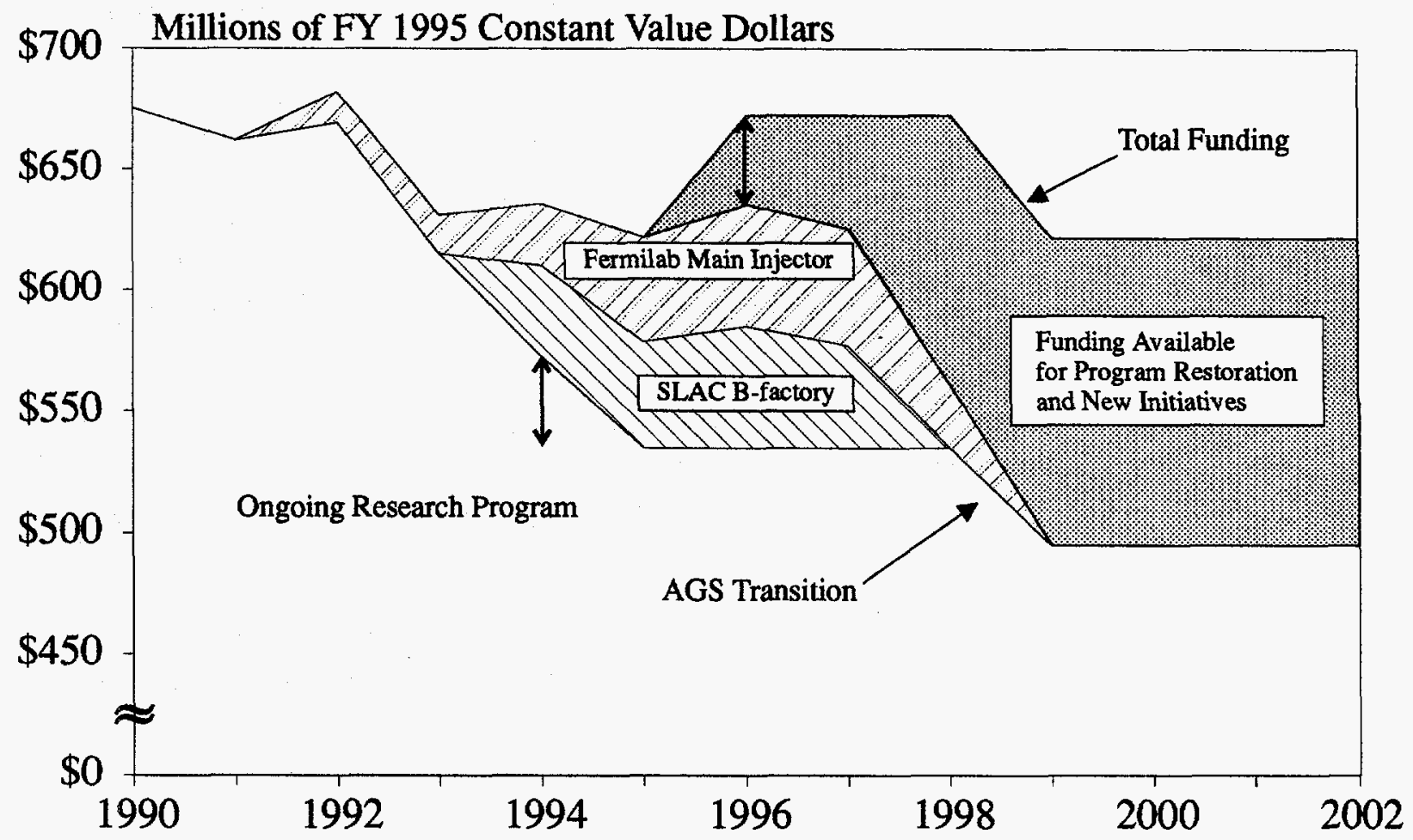

Fiscal Year

Figure 3. Same as Figure 2, but with suppressed zero. The total funding beyond FY1995 is set at the FY1995 level plus the proposed \$50M three year bump. The Fermilab Main Injector and the SLAC B-factory are shown on the basis of their approved funding profiles. The funding above that shown for the construction projects is available for program restoration and new initiatives. The two double-headed arrows indicate that the bump in FY1996 restores the loss in the ongoing research program between FY1994 and FY1995. 


\section{Recommendation 2}

The subpanel recommends that the federal government commit itself to a budget for the Department of Energy's High Energy Physics program that provides constant-level-of-effort funding plus a $\$ 50 M /$ year bump for three years, starting in FY1996, to implement the following program:

- Productive use of existing domestic facilities and their ongoing upgrades, including support for the university-based researchers, and flexibility to pursue new ideas.

- Significant participation in the LHC accelerator and detectors, both to provide research opportunities at the energy frontier and to ensure that U.S. physicists remain integrated in the international high-energy physics community.

- Enhanced effort in accelerator research and development, in preparation for a strong role in creating the accelerators of the next century.

\section{Recommendation 3}

Given the above three-year supplement and a commitment to support at no less than constant-level-of-effort funding thereafter, the subpanel recommends that the U.S. government declare its intention to join other nations constructing the LHC at CERN and initiate negotiations toward that goal. Participation in the LHC should be endorsed with a timely decision of support. This will enable the highenergy physics community in the United States to take full advantage of this opportunity and to maintain momentum in the collaborations that have been forming in the hope of applying to the LHC the expertise and technology developed for the SSC and its detectors, and of sharing in its discoveries.

The subpanel foresees U.S. expenditures for the LHC starting in FY1995, at the level of $\$(5$ to 10)M in FY1995, and $\$(10$ to 15$) \mathrm{M}$ in FY1996, with larger expenditures thereafter. Starting in FY1999, support of approximately $\$ 60 \mathrm{M}$ per year would result in a total of about $\$ 400 \mathrm{M}$ at the end of FY2003. We expect that a large fraction of this sum would be spent in the United States on building, for instance, special magnets and equipment for the interaction regions and the large detectors. (The figure of $\$ 400 \mathrm{M}$ is introduced simply to indicate the scale of possible total involvement under this budget assumption.) 
Such a commitment would constitute a serious, effective, and important U.S. investment of great value to both the U.S. and the Large Hadron Collider program.

The LHC is not only an important scientific opportunity, but also an important step in what we expect to be a growing trend in international collaboration on construction of large scientific instruments. The experience gained from this collaboration should thus strengthen the U.S. potential to be a host country for other international scientific endeavors.

As this discussion emphasizes, it is vital to have predictable funding for research projects that are large and costly multi-year commitments. This approach contrasts with recent history, in which such projects have been funded through the annual appropriations process, without prior Congressional authorization.

\section{Conclusion 4}

The subpanel emphasizes the importance of future major high-energy physics construction projects being fully authorized at the start of the project. This process, although it does not guarantee full funding by the government, can be important in building the support in Congress that is essential for the success of a large project. Full authorization at initiation of a construction commitment will bring needed strength to the role of the U.S. as a reliable partner prepared to undertake and complete long-term commitments. It can also help to ensure that projects proceed efficiently and expeditiously.

We also endorse the conclusion, emphasized in the report on "Science, Technology, and Congress: Organizational and Procedural Reforms" (February 1994) by the Carnegie Commission on Science, Technology and Government, that multi-year funding would greatly facilitate the planning of large projects and increase their operating efficiency. For the high-energy physics program, a budget cycle of two or more years would provide major advantages, because experiments depend primarily on large and complex instruments that take many years to build, and research generally involves long-term collaborations.

\section{Recommendation 4}

The government should give serious consideration both to restoring , earlier practices of full authorization at the start of major scientific construction projects and to introducing budget cycles of two or more years. 
The subpanel also considered implications of a flat, FY1995 constant-levelof-effort, budget without the supplement.

\section{Conclusion 5}

We emphasize that the main purpose of the temporary $\$ 50 \mathrm{M}$ budget bump is to strengthen the existing program. Without a three-year, $\$ 50 \mathrm{M} /$ year supplement in FY1996, the current U.S. program would suffer continued damage. The program's scope and flexibility would be further diminished, and ongoing commitments would be stretched out. This conclusion is independent of U.S. involvement in the LHC.

We do not believe that this problem should be addressed by continued proportional budget decreases at each of the laboratories and in each area of the program. We do believe that new priorities would have to be set that would likely call for sacrificing important parts of the U.S. program, in order to preserve quality and productivity in what survives. The inevitable consequences will be continued loss of vitality in the current program and further discouragement to the new generation entering the field.

As argued earlier, the commitment to initiate effective collaboration at LHC has little impact on the need of the current program for roughly $\$ 50 \mathrm{M}$, up front, to redress its needs. Also, as Figures 2 and 3 show, the planning assumption of a constant-level-of-effort budget for the future provides sufficient flexibility for effective LHC involvement. This budget scenario may necessitate some delays in making a sizable U.S. contribution to the LHC, and may reduce the total commitment, but it should not close the door. We must find a way to do the most important things; the LHC is one of them.

\section{Recommendation 5}

If there is no three-year, $\$ 50 \mathrm{M} /$ year bump in the budget, the subpanel recommends that the Department of Energy appoint a special subpanel of the High Energy Physics Advisory Panel to review the current U.S. high- energy physics program (preferably jointly with the National Science Foundation) and recommend appropriate changes and sacrifices.

However, the Subpanel still believes that joining the LHC collaboration is of sufficient importance that the U.S. should commit to doing so under a constant FY1995 level-of-effort budget, and should enter negotiations toward that goal. 


\section{Managing the Program}

The potential of the U.S. to make historic contributions in high-energy physics must be realized in a way that uses the public's money and other resources as efficiently as possible. The subpanel has identified aspects of the program that should be reviewed by the Division of High Energy Physics of the Department of Energy, in collaboration with the high-energy physics community. These include governance, within the field and by the Department of Energy, with regard to the setting of priorities, the phasing of construction, the balance of resources and representation between universities and laboratories, opportunities for young scientists with new ideas and initiatives, and rewarding superior scientific performance. Mechanisms for interagency support for the interdisciplinary field of particle astrophysics should also be reviewed.

The subpanel has not been in a position to focus on such issues, but we believe they deserve consideration by the Department of Energy, with National Science Foundation and community involvement. There is also need for an assessment of what the community perceives to be a growing administrative burden, beyond what is needed for proper accountability, and an excessively bureaucratic application of Environmental, Safety \& Health (ES\&H) regulations that is neither risk-based nor adequately evaluated for its contribution to safety.

\section{Using SSC Assets}

On behalf of the entire high-energy physics community, the subpanel wishes to acknowledge the extraordinary generosity of the State and the people of Texas in connection with the SSC. Although world science has now lost the SSC, it is vital to remember that not everything that went into the SSC has also been lost. Both intellectual and physical assets remain. The research and development that were carried out are still available to science and industry, through the literature and through the experience of many excellent scientists and engineers. Most of these people are now-or will soon be-applying their knowledge and experience in industry and in government laboratories, or engaged in research and teaching in universities or at the highenergy physics laboratories. The physical assets consist primarily of equipment on the Ellis County site.

\section{Conclusion 6}

The subpanel understands that a vigorous process for encouraging and reviewing proposals for on-site use of buildings and equipment is now proceeding under a cooperative agreement between the Department of 
Energy and the Texas National Research Laboratory Commission (TNRLC). These uses could be educational, medical, scientific, or commercial-or some combination of these. The subpanel applauds this effort to make good use of the on-site investment.

One possible use of the buildings and equipment is a superconducting magnet laboratory, which might be the center for U.S. participation in the CERN LHC project. The subpanel anticipates difficulties with such a use. The powerful cadre of scientists and engineers that came together to design, assemble, and operate the SSC has now been widely dispersed. Prospects for rebuilding and maintaining a scientific and technical staff of the highest caliber, far from a high-energy accelerator laboratory, are, in our judgment, not good.

\section{Recommendation 6}

Proposals for a scientific mission for the former SSC site should undergo stringent peer review. The review should call upon international experts in relevant areas of science to judge the proposals on their scientific and technical merit, feasibility, and costeffectiveness compared with other possible avenues for doing the same work.

Concerns about the vitality of a superconducting magnet laboratory for high- energy physics that is geographically separated from an accelerator laboratory will have to be weighed in evaluating proposals for such a laboratory on the former SSC site. The subpanel believes that these concerns weigh against such use.

Disposition of movable equipment will depend upon agreements between the Department of Energy and the State of Texas, which may involve the resolution of financial claims of the State. The subpanel does not presume to comment on issues outside our scientific and technical expertise in high-energy physics. However, if some of the equipment will not ultimately be used on site, it could be used elsewhere to the benefit of the U.S. high-energy physics program. The subpanel urges all parties to decisions regarding the disposition of SSC assets to recognize that a timely resolution will make it more likely that they will be put to good use. 


\section{In Conclusion}

We have presented a vision for the future in very general terms. It is too soon after the cancellation of the SSC to be more specific. At this stage, that is just as well: on the time scale of decades required to plan such large basic scientific endeavors at the frontiers of the unknown, we may expect big surprises. We have charted a path that, within its budgetary outlines, preserves a healthy balance and flexibility.

There is no way of predicting what scientific discoveries and technical breakthroughs may occur during the next decade. Such advances might lead us to seek to initiate another major international construction project before completion of the LHC. Additional funding would then be required. On the other hand, failure to maintain support for a constant level of effort in the future could deny the U.S. the opportunity to continue its tradition of success in the field of high-energy physics.

The U.S. high-energy physics program has long been a source of dramatic scientific progress and national pride, and a symbol of international collaboration. Building on the superb quality of its institutions of higher learning and research, and the generous support of the American public, the U.S. program has been a world leader at the frontiers of discovery. If our recommendations are implemented, we believe it will remain so well into the twenty-first century. 


\section{WHY TAKE THE NEXT STEP?}

Like all basic research, particle physics expresses the fundamental human passion for understanding the world around us. Through the ages, inquiring human minds have created a great scientific tradition and a beautiful structure of knowledge in the search to understand the nature and behavior of the matter of which the universe is made. Modern science in the twentieth century has inherited this tradition and, with the benefit of modern technology, has taken enormous strides forward. On the frontier of particle physics, progress has been nothing less than spectacular. Incisive experiments have revealed a realm that no one in all previous generations knew existed: the world of the elementary particles-the quarks and leptons-and the forces between them. Particle physicists have also contributed to a broader, multidisciplinary effort that has for the first time revealed a glimpse of the history of how our universe has evolved since the Big Bang some 15 billion years ago.

And progress is being made at this very time on the frontier of particle physics. As this report was being prepared, the first evidence was presented from an experiment at the Fermi National Accelerator Laboratory (Fermilab) that there exists a massive top quark, the long-sought sixth member of this family of elementary particles.

To investigate nature on this infinitesimal scale, so small that it cannot be seen with even the most powerful microscope, scientists have devised extraordinary techniques. Particle physicists start with a beam of very highenergy particles, often from a machine built expressly to accelerate a particular kind of particle to a specified energy. When a particle in the beam collides with another particle of matter, insights can be gained into the structure of matter and the forces that govern its behavior by studying the pattern of particles emerging from the collision. These particles are recorded by the devices known as detectors.

Today, high-energy accelerators can supply particles with sufficient energy to behave as they did in the very hot, very dense conditions of the early universe. Experiments can therefore be performed in which particles are actually created, converting energy into mass according to Einstein's famous equation, $\mathrm{E}=\mathrm{mc}^{2}$. Although the notion of creating matter may be unfamiliar, that is only because conditions on Earth are too cold for it to occur here and now. Whenever two particles collide at high energies, nature dictates which new particles can be created and how one form of matter can be transformed into another. Whether the collision occurred when the universe was less than a second old or yesterday at an accelerator laboratory, the same laws of 
particle physics hold for the behavior of matter at any time and place in the universe. Thus, experiments done now on Earth can tell us much about events far removed from us in distance and time.

One of the wonders of this world is that the particles and forces at this almost unimaginably small scale can have such profound consequences for us. For example, consider the mass of the electron: if it had turned out to be somewhat more massive, would anything be different from the way it is today? Extraordinarily so. In contrast to the world we know, it might well have been possible for such massive electrons to be captured by the protons. In that case, after the Big Bang, the universe would have consisted entirely of neutral particles. There would have been no protons, hence no nuclei, atoms, stars, or planets, hence no human beings.

Discovering and fitting together the pieces of this extraordinary puzzle is part of the adventure of particle physics. As particle physicists have peered deeper into the heart of matter, their questions have changed. Today's research agenda includes trying to understand why particles have the masses they do, why there is more matter than antimatter in the universe, and what makes up the 90 percent of the matter in the universe that we know exists but is now invisible to us.

Another important challenge is figuring out how to explore these questions. Particle physicists cannot simply call up a supply company and order a high-energy accelerator or a detector. They must be intimately involved in the design and construction of their instruments. Research in fundamental aspects of accelerator and detector technology, and addressing the technical issues associated with a particular design, flow smoothly into actual construction and use. All are part of doing particle physics. And-in part because these instruments lie beyond the reach of single universities, states, and sometimes even nations, and in part because science provides a common language and a common vision-the quest to understand the universe at this level has brought together men and women from widely different social, national, and ethnic backgrounds in fruitful and satisfying collaborations.

The achievements of particle physics belong to all of science, and to all who are curious about the universe in which we live. Every American can take a justifiable pride in the contributions that science in the United States has made to our country and to humanity. 
In particle physics, these include first observations of the heavy quarks, discovery of the breakdown of symmetry in the physical laws governing matter and antimatter, and the recognition that the electromagnetic force and the force governing radioactivity are simply different forms of one single, underlying force.

In addition, particle physics addresses questions that capture the imagination of students, drawing them into science. Some eventually pursue scientific careers in particle physics or in other fields; all benefit from the analytical, imaginative thinking that is at the core of science.

Particle physics, in common with all basic scientific research, also contributes to the rest of society by harnessing innovative technologies. The technological base of cancer radiation therapy is the electron linear accelerator, adapted by researchers in other fields from the device originally developed at Stanford University for high-energy physics research; one out of eight people in the United States will receive radiation treatment at a linear accelerator sometime in his or her life. The need for enough reliable superconducting magnets to fill a tunnel four miles long to build the Tevatron collider at Fermilab was a cornerstone of commercial development of the superconducting cable now used in MRI machines. The challenge of very rapidly analyzing vast amounts of data from particle physics experiments has contributed to advances in cost-effective high-speed computing and communications. In fact, particle physicists invented the World Wide Web to share vast amounts of data among collaborators around the globe.

The loss of energy suffered by a charged particle accelerated along a curved path was long regarded as a nuisance by high-energy physicists. However, researchers in other fields eventually saw a way to put this so-called synchrotron radiation to good use. X-rays and ultraviolet light from accelerators known as synchrotron light sources provide valuable information about atomic and molecular structure, chemical composition, the dynamics of structural transition, and the magnetic properties of matter, which benefits the petrochemical, pharmaceutical, semiconductor, and computer industries. Accelerators can also play a role in protecting and restoring the environment, by, for example, supplying pulsed electron beams to reduce the toxicity of chemical wastes.

The development of the free electron laser (FEL) is a good example of the close intertwining of science, invention, and technology, and of the shared benefits that often result from such a close relationship. The product of electron beam and laser technology, the FEL is just starting to influence surface science, biology, chemistry, and medicine. 
There is a rich, historical record of fundamental new knowledge opening possibilities to a better way of life. But we are not wise enough to know how to target basic research to ensure particular technological advances. Who could have predicted that the development of quantum mechanics to explain the behavior of atoms in the 1920s would have a crucial role to play, decades later, in the development of the transistor, the semiconductor, and it is likely, the nanotechnologies of the future? In 1911, when the phenomenon of superconductivity was discovered, who would have anticipated the benefits to basic research and to medicine of the innovative technologies it would make possible half a century later? As Vice President Gore said, in a speech to the Office of Science and Technology Policy Forum, (February 1, 1994), "We cannot afford to take the narrow view of science-looking only at immediate results. We have to cast our eyes ahead a few years, a few decades, a century or more, and imagine the unimaginable."

Our country has long cherished the pursuit of knowledge for its own sake. That has been one of our strengths. At the same time, better comprehending the world around us has throughout history been at the core of improved productivity, economic vitality, and technological innovation. The ability to do basic research at the frontiers of science, and to do it together with people from around the world, is a vital legacy to future generations. Understanding the structure of matter and the fundamental forces will undoubtedly stand as one of humanity's finest achievements. As a great nation, the United States can-and should-play a leading role. 


\section{ELEMENTS OF A WORLD-CLASS PROGRAM IN HIGH-ENERGY PHYSICS}

The U.S. high-energy physics program has long been a source of important scientific progress and national pride. Building on the superb quality of American institutions of higher learning and the generous support of the American public, the U.S. program has been a world leader at the frontiers of discovery. In the United States there are roughly 2,500 researchers. The majority are located at universities where they teach and train undergraduate and graduate students in addition to their research, which is pursued primarily at the national laboratories. In addition there are approximately 1,100 graduate students.

Over the past decade, the highest priority of the U.S. high-energy physics program was to design and build the Superconducting Super Collider (SSC). This commitment was based on extensive analyses of the most important scientific questions and of the practical opportunities for advances in accelerator and detector technologies. That path to the future was closed in the past year by the congressional decision to terminate the SSC project. (See Appendix A.) Consequently, the U.S. high-energy physics community must now develop a new strategy, a new path to the future, that is at one and the same time:

- scientifically compelling,

- affordable and acceptable to our government and the public, and

- consistent with the U.S. remaining among the world leaders in this field of fundamental science.

In this chapter, we take the first step in developing the new strategy by discussing five key elements of a world class program in high-energy physics. In Chapter VIII, we face the challenge of matching the scope and size of a proposed program that incorporates these elements to budgetary realities.

A. We must ensure that the U.S. program is responsive, flexible, dynamic, and progressive.

By its very nature, basic research in science explores unknown realms. General principles and patterns of understanding guide the search, but history provides much evidence-in fact history almost guarantees-that we will encounter surprises. These will lead to new insights and motivate new directions of research out of which new paradigms will emerge. Wise planning for the future should avoid 
focusing too narrowly on one definite goal or means of inquiry, no matter how strongly motivated it may appear to be at present.

Wise planning will also ensure support for a variety of research opportunities along different paths. These include studying the new phenomena revealed by accelerated beams of particles reaching higher energies; looking for very rare phenomena with intense beams of particles; making precision measurements in search of small but significant deviations from theoretical predictions; and exploring issues that accelerators cannot address, by studying particle cosmology, neutrino and ultra-high-energy astrophysics, for example. To emphasize the great diversity of important phenomena and methods of inquiry, as we seek to understand the fundamental building blocks and forces, we often speak of particle physics instead of high-energy physics. Both are asking the same questions.

B. We must be able to attract, train, and provide opportunities to young scientists.

Particle physics inspires bright young minds because the issues it addresses are challenging and compelling. But without opportunities to conduct productive, timely research on the most provocative issues and to advance into leadership positions, they will seek fulfillment in another field, or outside science altogether.

While training in particle physics prepares students well for rewarding and productive careers in other fields (the path that between $60 \%$ and $70 \%$ of high-energy physics graduate students have followed over the past decade), it is also essential that particle physics retain its full share of the best young talent, if our future is to be as bright as our past.

C. There must be a balance between the "ongoing" program and the "future" program.

As particle physicists have pushed back the frontier of understanding, new questions have been raised that require more powerful scientific instruments. Designing and building large, complex accelerators and detectors can now take a decade or more. These time scales present an especially difficult challenge in planning a healthy, balanced research program with realistic assumptions about available resources. The fruits of investments already made must be gathered in an ongoing program that advances our understanding. At the same time, investment in longer term research and development is necessary to 
search for creative, practical, and affordable new technologies for detectors and accelerators that will open new frontiers for the future program. In between the ongoing and future programs, there is a continuum of activity, including improvements to existing accelerators and detectors to ensure near-term progress. These are all important aspects of a properly balanced and unified research program.

D. We should now be developing more extensive collaborations for designing and constructing any new multi-billion dollar accelerators.

With our increased understanding of elementary particles and their interactions, the questions we can ask have become more profound, and the machines required to answer them have become larger, sometimes beyond the reach of any single nation. To continue to make progress, we will need to develop more extensive international collaboration.

E. We need predictable funding.

The strong, stable funding base and enlightened public support that have made possible our past triumphs in high-energy physics are essential for future success. A steady funding base is particularly critical as we enter an era in which large construction projects require multiyear commitments and international collaborations become increasingly important, even prevalent. Large new construction projects for the future must reflect a commitment on the part of both the scientists and the governments involved that pledges of talent and resources be firm and realistic, to ensure proceeding expeditiously and efficiently to completion. 
(BLANK) 


\section{ELEMENTARY PARTICLE PHYSICS TODAY}

\section{A. INTRODUCTION}

High-energy physics is the search for elementary particles and basic laws of nature. What are the smallest building blocks out of which protons, neutrons, atoms, and all matter are made? Do such elementary particles exist?; and if so, what are they? This search to unveil the elementary constituents of matter, along with the forces that link them, involves distances thousands of times smaller than nuclear sizes, about one ten trillionth of a centimeter, or $10^{-13} \mathrm{~cm}$. Accelerators must have very large energies to probe nature at such small distances. The ultimate goal of this quest is a view of the underlying first principles that govern our entire physical universe.

In recent years, we have realized a strong and growing synergism between the physics of short distances and the properties and largescale structure of the universe. This development reflects the unity of science as explored on both the high-energy and particle astrophysics frontiers. With this connection, we are now addressing some of the most basic questions one can ask: How did our physical universe begin? How did it evolve to its present state? What will be its final fate?

Over the past several decades, experimental discoveries and theoretical insights have significantly advanced our understanding of the elementary particles and their forces. We now know that electrons, protons, and neutrons make up the visible matter all around us, but only the electron appears to be a point-like elementary particle. Protons and neutrons are bound states of more basic constituents, the up and down quarks. Those quarks are permanently bound or confined by what are called strong interactions or forces.

The strong interactions are governed by a fundamental theory of quarks and gluons known as quantum chromodynamics (QCD). The gluons mediate the strong force that binds the quarks into protons and neutrons. QCD is an elegant theory that, in principle, is capable of explaining all observed strong interaction physics.

On another front, two forces previously thought to be distinct, electromagnetism and the weak force that governs radioactive decay, are now properly described by a unified electroweak theory. This theory correctly predicted weak neutral currents as well as the observed properties of $W$ and $Z$ bosons, the carriers of the weak force and partners of the photon. 
The combination of QCD and the electroweak model provides a beautiful description of all known elementary particles down to distances of order $10^{-16} \mathrm{~cm}$. The theory of strong and electroweak interactions can be unambiguously tested by comparing its predictions with precision measurements. Remarkably, a wealth of experimental data has been confronted at a high level of sensitivity, without any clear signal of disagreement or inconsistency. Those impressive successes have earned the theory its title as the "Standard Model," a label that describes its acceptance as a proven standard against which future experimental findings and alternative theories must be compared. Its discovery should be viewed as one of the great scientific triumphs of the twentieth century.

Despite the successes of the Standard Model, it is believed not to be the final word. That conviction is based primarily on dissatisfaction with the electroweak sector which exhibits a number of shortcomings and leaves unanswered some basic questions: Why are there so many elementary particles and why do they have their observed pattern of masses? What is the origin of mass? Why and how is the symmetry between electromagnetism and weak interactions broken? Why is matter-antimatter symmetry broken and what does it have to do with the observed predominance of matter in our universe? Speculations abound, but physics is an experimental science, and only with new data will we be able to properly address these problems and uncover whatever new surprises lie ahead.

\section{B. THE STANDARD MODEL}

As an outline of the Standard Model, we have illustrated in Table A its spectrum of elementary particles, along with some of their basic properties [including their electric charge, their spin, and their mass, expressed in units of one billion electron volts $(\mathrm{GeV})$, which is roughly the mass of a proton]. The fermions are grouped into three generations with remarkably similar features. Indeed, the masses of the quarks and leptons represent the only significant difference between the generations.

The first generation contains the constituents of ordinary matter. The second and third include heavy unstable elementary particles, which can only be studied in high-energy processes. Indeed, a remarkable feature of the theory is that the elementary constituents can transform into one another according to well-defined rules. It now appears that elementary particles are fundamental but not immutable, in contrast to the views of many early Greek philosophers. 
The neutrinos are massless in the minimal Standard Model. Although this prediction is consistent with experiments to date, there are some tantalizing hints of tiny neutrino masses from solar and atmospheric experiments. (The sun and upper atmosphere are copious sources of neutrinos.) Should nonzero neutrino masses be established, they could be accommodated into theory, but they would likely be a signal of new physics. In fact, many attempts to synthesize the strong and electroweak forces into a grand unified theory naturally predict very small neutrino masses.

Table A: Elementary Particles and Their Properties

First Generation Fermions

\begin{tabular}{|l|c|c|c|c|}
\hline \hline Particle & Symbol & Spin & Charge & Mass (GeV) \\
\hline Electron Neutrino & $v_{\mathrm{e}}$ & $1 / 2$ & 0 & $<7.2 \times 10^{-9}$ \\
\hline Electron & $\mathrm{e}$ & $1 / 2$ & -1 & $0.51 \times 10^{-3}$ \\
\hline Up Quark & $\mathrm{u}$ & $1 / 2$ & $2 / 3$ & $\sim 5 \times 10^{-3}$ \\
\hline Down Quark & $\mathrm{d}$ & $1 / 2$ & $-1 / 3$ & $\sim 9 \times 10^{-3}$ \\
\hline
\end{tabular}

Second Generation Fermions

\begin{tabular}{|l|c|c|c|c||}
\hline \hline Particle & Symbol & Spin & Charge & Mass (GeV) \\
\hline Muon Neutrino & $v_{\mu}$ & $1 / 2$ & 0 & $<2.7 \times 10^{-4}$ \\
\hline Muon & $\mu$ & $1 / 2$ & -1 & 0.106 \\
\hline Charm Quark & $\mathrm{c}$ & $1 / 2$ & $2 / 3$ & $\sim 1.35$ \\
\hline Strange Quark & $\mathrm{s}$ & $1 / 2$ & $-1 / 3$ & $\sim 0.175$ \\
\hline
\end{tabular}

Third Generation Fermions

\begin{tabular}{|l|c|c|c|c||}
\hline \hline Particle & Symbol & Spin & Charge & Mass $(\mathrm{GeV})$ \\
\hline Tau Neutrino & $v_{\boldsymbol{r}}$ & $1 / 2$ & 0 & $<3 \times 10^{-2}$ \\
\hline Tau Lepton & $\tau$ & $1 / 2$ & -1 & 1.78 \\
\hline Top Quark & $\mathrm{t}$ & $1 / 2$ & $2 / 3$ & $174 \pm 17$ \\
\hline Bottom Quark & $\mathrm{b}$ & $1 / 2$ & $-1 / 3$ & $\sim 4.5$ \\
\hline
\end{tabular}


Gauge Bosons

\begin{tabular}{|l|c|c|c|c|}
\hline Particle & Symbol & Spin & Charge & Mass (GeV) \\
\hline Photon & $\gamma$ & 1 & 0 & 0 \\
\hline W Boson & $\mathrm{W}$ & 1 & \pm 1 & 80.22 \\
\hline Z Boson & $\mathrm{Z}$ & 1 & 0 & 91.19 \\
\hline Gluons & $\mathrm{g}$ & 1 & 0 & 0 \\
\hline
\end{tabular}

Higgs Boson

\begin{tabular}{|l|c|c|c|c|}
\hline Particle & Symbol & Spin & Charge & Mass $(\mathrm{GeV})$ \\
\hline Higgs Boson & $\mathrm{H}$ & 0 & 0 & $63<\mathrm{M}_{\mathrm{H}}<800$ \\
\hline
\end{tabular}

The study of the top quark and its properties represents an exciting frontier for particle physics. Ongoing experiments at the Fermi National Accelerator Laboratory (Fermilab) Tevatron have recently produced the first direct evidence for the top quark, and indicate that its mass is $174 \pm$ $17 \mathrm{GeV}$, making it much heavier than any other known elementary particle. Why is the top quark so heavy? This question highlights the broader question of why nature chose to repeat the fermion generation structure three times and endow quarks and leptons with their observed pattern of masses. Understanding the mass spectrum of elementary particles is an outstanding problem for high-energy physics. Perhaps the very large top quark mass, relative to all the other quarks, holds the key to solving that problem.

Quarks and leptons interact by exchanging spin-one particles known as gauge bosons. The best known gauge boson is the photon that mediates electromagnetism. Its electroweak partners, the $W$ and $Z$ bosons, mediate the weak forces. The large masses of the $W$ and $Z$ in Table A stand in sharp contrast to the masslessness of the photon.

The masses of the electroweak gauge bosons indicate the degree by which the symmetries of nature are broken. At very short-distances or high energies, the $W, Z$, and photon have similar properties and the symmetries among them are manifest. At large distances, the symmetry is broken and the photon is preeminent. As a result, electromagnetism controls most of the physics and chemistry of everyday life. 
The massless gluons of QCD mediate the strong interactions. Quantum chromodynamics has no free parameters; it is capable in principle of predicting the masses of all hadrons (i.e. the proton, neutron, rho meson, etc.) as well as nuclear properties and scattering cross-sections. It is the fundamental theory that underlies the more phenomenological models appropriate for nuclear physics. In fact, lowenergy particle physics is hard to distinguish from nuclear physics, and cross-disciplinary collaborations have helped to address common questions.

Calculations in QCD from first principle are extremely difficult because its interaction between quarks and gluons is so strong. Nevertheless, using techniques borrowed from condensed matter physics, theorists are tackling some of these problems with the world's most powerful computers. Now that a complete theory of strong interactions appears to be in hand, the challenge is to fully explore and understand its dynamical properties and subtle features. Who knows what surprises it may yet hold?

\section{ELECTROWEAK SYMMETRY BREAKING}

In contrast to quantum chromodynamics, the description of electroweak processes in the Standard Model has many arbitrary or free parameters. Most stem from the breaking of the underlying symmetry between electromagnetism and the weak interactions. This symmetry breaking provides mass for the $W$ and $Z$, but leaves the photon massless.

In the minimal Standard Model, electroweak symmetry is broken by the Higgs mechanism. This idea has its roots in condensed matter physics, where it was introduced in connection with the LandauGinsberg theory of superconductivity. In this scheme, a particle's mass depends on its interactions with the Higgs field, a medium that permeates all of space and time. The $W$ and $Z$ masses result from their couplings to this field. The photon and gluon have no such couplings, so they remain massless.

Quark and lepton masses are determined by the strength of their couplings to the Higgs field. These couplings also determine the extent to which quarks can mix between generations. Even charge parity $(\mathrm{CP})$ violation- $a$ fundamental asymmetry between matter and antimatter that may be responsible for matter dominance and our place in the universe-is generated by couplings to the Higgs. Unfortunately, we do not understand the origin of these couplings, so they must be 
determined phenomenologically by experiments. Current theoretical models can accommodate a top quark mass 340,000 times that of the electron and the small degree of $\mathrm{CP}$ violation seen in nature, but we cannot explain them.

\section{THE Higgs PARTICLE}

A testable prediction of the minimal Standard Model is the existence of a neutral spin-zero elementary particle $\mathrm{H}$ called the Higgs boson, associated with the Higgs field. The Higgs boson mass is, however, not predicted. The lower bound in Table 1 is determined by experimental searches and the upper bound is based on theoretical arguments.

If the $\mathrm{H}$ is too heavy, it is unlikely to exist as an elementary particle. Instead it is more likely to be replaced by a new set of strongly interacting dynamics. At present, there is no experimental evidence in favor of a Higgs particle, nor is there any against. Finding the Higgs boson, or whatever takes its place, is crucial for understanding and going beyond the physics of the Standard Model.

Although introducing a Higgs field provides a simple mechanism for electroweak symmetry breaking, we really do not understand at a deep level why this phenomenon occurs. In fact, the Higgs mechanism with its concomitant spin-zero Higgs boson has a variety of theoretical shortcomings. The model on which it is based is unstable against quantum corrections when embedded in a theory of gravity or grand unified theory. In addition, although the simplest Higgs model can accommodate all known particle masses, mixings, and even $\mathrm{CP}$ violation, it does not explain their origin.

Even though our knowledge of electroweak symmetry breaking is incomplete, the mass values of the $\mathrm{W}$ and $\mathrm{Z}$ bosons identify the energy scale where this phenomenon becomes manifest. Irrespective of what is the precise agent that causes the symmetry breakdown, we believe that the physics which underlies it will be uncovered when we will be able to thoroughly probe matter at this energy scale. Through experimentation at much higher energies than those presently available we hope that a truly fundamental understanding of electroweak symmetry breakdown will emerge that will elucidate the origin of mass through additional symmetry, new dynamics, or by some as yet unknown phenomenon. Uncovering those missing ingredients and deciphering their role was a major focus of the Superconducting Super Collider (SSC), and remains one of the most important goals of highenergy physics today. 
Another outstanding problem in elementary particle physics is the very small asymmetry between the properties of matter and antimatter (particles and antiparticles), related to $C P$ violation. When first observed in a 1964 Brookhaven National Laboratory (BNL) experiment, this asymmetry came as a complete surprise. Since then we have learned that $\mathrm{CP}$ violation is a necessary ingredient for explaining the dominance of matter over antimatter in our universe.

The origin of $\mathrm{CP}$ violation remains mysterious to this day. Within the framework of the Standard Model, CP violation can be accommodated through quark mixing effects. Such mixings give testable predictions that are being studied in $K$ meson decays and will be further scrutinized in $B$ decays.

The Standard Model, however, does not really explain the underlying reason for $\mathrm{CP}$ violation. Furthermore, it appears that an additional source of $\mathrm{CP}$ violation from some as-yet-undiscovered new physics may be necessary to explain the matter-antimatter asymmetry of our universe.

Testing the Standard Model's description of this phenomenon and searching for non-standard CP violation are major goals of high-energy physics. Following that path may lead us to an understanding of the origin of mass and our universe.

F. BEYOND THE STANDARD MODEL

Many of the elements of the simple Higgs mechanism for electroweak symmetry breakdown can be retained if an additional symmetry between bosons and fermions, called supersymmetry, were to exist. This elegant symmetry alleviates quantum instabilities in the theory, at the expense of introducing a host of new elementary particles at masses near $1 \mathrm{TeV}$.

In supersymmetric theories, essentially every particle in Table A has a supersymmetric boson or fermion partner. Currently, supersymmetry has no direct experimental support; however, supersymmetric grand unified theories correctly predict low-energy coupling strengths. Additional strong motivation for supersymmetry is provided by superstring theories, which unify the Standard Model and gravity by replacing point particles with tiny strings. 
Many supersymmetric theories, furthermore, predict the existence of heavy, stable, neutral particles that have the potential to explain the missing mass of the universe. Astronomical observations indicate that visible objects might comprise less than $10 \%$ of the total mass of the universe. With its plethora of new particles, supersymmetric theories can solve this problem. If true, this would have profound implications for our place in the universe: we would not be made of the material that comprises the bulk of the universe!

Alternative to an elementary Higgs particle is dynamical symmetry breaking via fermion-antifermion interactions. This is analogous to the Bardeen-Cooper-Schrieffer theory of superconductivity in which electron-electron Cooper pairs replace the scalar order parameter of the Landau-Ginsberg phenomenological theory. Scenarios for electroweak symmetry breaking along these lines range from minimal top-antitop interactions to more ambitious schemes modeled on QCD. These models often predict many new heavy particles below the $\mathrm{TeV}$ scale.

Although the basic premise of these speculations is very appealing, no complete dynamical theory currently exists. We do, however, expect that new particles or interactions should appear, at a mass scale below a few $\mathrm{TeV}$. To make headway in unfolding dynamical symmetry breaking will require accelerators of the highest possible energy to discover new heavy fermions and bosons or some complete surprise. Such discoveries would provide the clues necessary to help guide our imaginations about the underlying dynamics.

In addition to supersymmetry and dynamical symmetry breaking, there have been many other possible suggestions for new physics. They include: extended symmetries with additional heavy gauge bosons $W^{\prime}$, $Z^{\prime}$, neutrino masses and associated oscillations among the three different species, new sources of $\mathrm{CP}$ violation, grand unification of strong and electroweak interactions, etc. The menu of possibilities is rich. Full exploration will require a diverse and broad-based experimental program that utilizes accelerator and non-accelerator facilities. Theorists may speculate, but data rules supreme in the study of nature.

\section{G. SEARCHING FOR NEW PHYSICS}

Testing the Standard Model and probing for new phenomena at accelerators can be roughly categorized by three approaches: high energy, high precision, and high intensity. The most direct way to find new physics is to go to higher energy and explore completely uncharted territory. The Fermilab Tevatron currently has the highest center-of-mass 
energy of any accelerator in the world. It is the only existing facility where top quarks can be produced and where there still remains the possibility that other new high-mass phenomena might be discovered. The Main Injector upgrade will increase the Tevatron's intensity and allow a better look at the top quark's properties. Pushing the highenergy frontier ever forward is the lifeblood of elementary particle physics.

Beyond the Tevatron, one must take large enough steps to ensure a significant new discovery potential. In that regard, the SSC energy of 40 $\mathrm{TeV}$ represented a factor of twenty increase over the Tevatron, and was chosen to allow thorough exploration of electroweak symmetry breaking, including discovery of the Higgs over its entire mass range.

The European Laboratory for Particle Physics' (CERN) Large Hadron Collider ( $\mathrm{LHC}$ ), with an energy of $14 \mathrm{TeV}$, represents a significant step beyond the Tevatron on the energy frontier. Although the LHC is not as energetic as the SSC, it has considerable discovery potential. A TeV-scale electron-positron collider would also extend our discovery potential and would be well-suited for thorough investigations of new phenomena.

Complementary to high-energy searches are high precision studies of the Standard Model. In this approach, one tests the consistency of standard-model predictions through precision experiments. Such studies allow us to refine our understanding of the Standard Model. In addition, any deviation from expectations would indirectly signal the presence of new physics.

Examples of precision measurements include the $W$ and $Z$ masses, the electroweak mixing angle, as well as the quark mixing angles. Of particular importance are plans to measure the $W$ mass to an accuracy of about $50 \mathrm{MeV}$ (better than $0.1 \%$ ) both at the Tevatron with the Main Injector upgrade, and at LEP II, along with the ongoing effort at SLAC to measure the electroweak mixing angle with similar accuracy using polarized electrons.

The third means of testing the Standard Model and hunting for new physics involves studies of very rare, or even forbidden processes, including $\mathrm{CP}$ violation. At accelerators, such experiments require high intensity. Traditionally, the $\mu$ and $K$ mesons have been used because of their relatively long lifetimes and copious production rates. Indeed, $K$ decays presently provide our only evidence for $C P$ violation. They also indirectly probe for new physics at the $200 \mathrm{TeV}$ scale, a domain well beyond the reach of our highest energy accelerators. Ongoing 
experiments at BNL and Fermilab continue to push the search for rare $K$ decays to unprecedented levels and probe for the origin of $\mathrm{CP}$ violation.

Rare decays of the bottom and charm quarks as well as the tau lepton are starting to reach significant limits. For example, the CLEO collaboration at CESR recently found the first evidence for rare radiative $b$ quark decays. Studies of $B$ mesons (that contain $b$ quarks) are particularly exciting because they open a new window to $\mathrm{CP}$ violation. Indeed, the standard model of $\mathrm{CP}$ violation predicts relatively large effects in $B$ decays. Studies of these predictions will be possible at highluminosity electron-positron $B$ factories as well as at high-energy hadron colliders.

Other examples of exotic phenomena that require high rates or massive detectors include neutrino oscillations from one type to another, non-standard CP violation searches and proton decay. Proton decay experiments are particularly impressive because they are our most direct window to physics at the grand unification mass scale. Indeed, present bounds on the proton lifetime already test physics at $10^{15} \mathrm{GeV}$. A joint Japan-U.S. experiment presently under construction at the Kamioka mine in Japan should push the proton lifetime search more than a factor of ten. Discovery of any reaction forbidden by the Standard Model would revolutionize physics and open up many new avenues of investigation.

A well-balanced experimental program must include this threepronged approach of high energy, high precision, and high intensity experiments, along with a variety of complementary non-accelerator initiatives. Only in that way can we hope to broaden our frontiers and increase our chances for discovery. What then are the most compelling questions and issues which currently drive our experimental program in high-energy physics and how can they be best addressed? As representative of the many exciting questions still to be answered by particle physics we propose the following list and briefly indicate with what facilities these questions may be answered.

\section{H. COMPELLING QUESTIONS}

Top Quark Physics: What is the precise value of the top quark mass? Why is it so heavy? What are its properties?

The Fermilab Tevatron is currently the only accelerator in the world capable of directly exploring top quark physics. The LHC, when commissioned about a decade from now, will produce many millions of 
top pairs per year, making it a veritable top factory. An electronpositron collider with energy just beyond twice the top mass would provide a clean environment for measuring top quark properties.

Electrowveak Symmetry Breaking: Is there an elementary Higgs boson? Is it part of a supersymmetry scenario? How do we uncover the Higgs boson and explore its properties? Alternatively, is the electroweak symmetry broken dynamically?

The LHC offers the opportunity to search for an elementary Higgs boson over the broad range of masses between 80 and $800 \mathrm{GeV}$. It can also explore extended Higgs models as suggested by supersymmetry. To understand all possible Higgs particles in this case, however, it would be important to also have access to a high-energy, high-luminosity electron-positron collider. There are scenarios in which the LHC discovery potential is limited and higher energy is required. Dynamical symmetry breaking would be such a case where the LHC's success would depend on the physics. In this case one might need a higherenergy hadron collider, with a broad-band discovery potential at least as great as that of the SSC.

Fermion Masses, Mixings, and CP Violation: What is the underlying physics of fermion mass generation? Can we test standard-model predictions for quark mixing and $\mathrm{CP}$ violation?

Whatever generates fermion masses apparently couples most strongly to heavy quarks, so it is very important to study the properties of the top and bottom quarks. $K$ and $B$ decays offer the best means of measuring the quark mixing parameters and refining our understanding of standard-model CP violation. Searches for very rare or even forbidden decays are a sensitive probe of the underlying physics of mass generation. With the BNL and Fermilab fixed-target programs, the Tevatron Collider, CESR at Cornell and the SLAC B-factory, the U.S. is well-positioned to study the physics of quark masses and the origin of $\mathrm{CP}$ violation.

Neutrino Masses and Mixings: Do neutrinos have nonzero masses? Are they part of dark matter? Do neutrinos oscillate from one type to another? 
Neutrino masses and oscillations can be studied using accelerator, reactor, solar, or atmospheric neutrino sources. Exploring the full panoply of neutrino masses and mixings probably will require both long and short baseline neutrino oscillation experiments, as well as beta decay studies, necessitating both accelerator and underground facilities.

QCD Dynamics: What is the structure of the proton? Can we better understand quark confinement? Are there exotic bound states? What is the precise value of the strong coupling constant?

Full exploration of QCD and its properties requires studies of nucleon structure, high-energy scattering, and searches for new forms of matter. Monte Carlo computer simulations provide a powerful means of investigating $Q C D$ properties. The study of QCD dynamics overlaps strongly with the future nuclear physics programs at the Continuous Electron Beam Accelerator Facility (CEBAF) and the Relativistic Heavy Ion Collider (RHIC), while important studies of QCD structure functions are underway at the Hadron-Elektron-Ring-Anlage ( HERA) accelerator in Hamburg, Germany, as well as at SLAC and Fermilab.

Electroweak Parameters and Quantum Corrections: What are the precise values of electroweak masses and couplings? Can we observe quantum loop effects?

Present precision electroweak experiments range from low energy studies such as atomic parity violation and anomalous magnetic moments to $Z$ studies at SLAC and CERN and $W$ mass measurements at Fermilab. A high-energy, high-luminosity electron-positron collider can make precision measurements of the gauge-boson interactions and open a window to physics well beyond the energy of the machine.

Supersymmetry: Is supersymmetry manifest at or below $1 \mathrm{TeV}$ ? If so, can we uncover the supersymmetric spectroscopy? Do supersymmetric particles contribute to the missing mass of the universe?

The LHC is capable of finding signals for supersymmetry up to mass scales of about $1.5 \mathrm{TeV}$. Full exploration of the supersymmetric spectrum can be accomplished by an electron-positron collider with sufficient energy to pair-produce the supersymmetric particles. Underground searches for dark matter could also uncover such particles. 
Additional Gauge Bosons: Are there $W^{\prime}$ and $Z^{\prime}$ bosons? How can we find them?

Direct production of $W^{\prime}$ or $Z^{\prime}$ bosons requires high-energy colliders. The LHC, for example, can search up to about 3 to $4 \mathrm{TeV}$, while a TeV electron-positron collider can indirectly probe similar scales and would provide constraints on the gauge symmetry of the new interaction. Lowenergy experiments such as those on atomic parity violation and polarized electron scattering can also indirectly provide evidence for $Z^{\prime}$ bosons via deviations from Standard Model predictions.

Non-Standard CP Violation: Is there $\mathrm{CP}$ violation beyond the Standard Model? Is it related to the matter-antimatter asymmetry of the universe?

Searches for electric dipole moments and $\mathrm{CP}$ violating asymmetries such as the transverse muon polarization in $K^{+} \rightarrow \pi^{0} \mu^{+} v$ decay are examples of experiments that can be sensitive to $C P$ violation beyond the Standard Model. A full program of $C P$ violation studies in $B$ and $K$ decays will probe not only standard-model predictions, but could uncover, through precision studies, a new source of $C P$ violation.

Grand Unification: Can we confirm a grand unification of strong and electroweak interactions? Can we observe proton decay? Magnetic monopoles? Can we test supersymmetric unification? String theory?

Super-Kamiokande offers an opportunity to push searches for proton decay more than an order of magnitude beyond current bounds, to within the range predicted by some supersymmetric theories. It is also capable of studying solar and atmospheric neutrinos and searching for magnetic monopoles from grand unification.

Although the Standard Model provides an apparently complete description of particle physics at present energies, and answers many questions, it gives rise to many more. With a vigorous, broad-based program on the energy, intensity and precision frontiers, we can look forward to great progress during the coming years. 
(BLANK) 


\section{THE EXISTING ACCELERATOR-BASED PROGRAM}

\section{A. INTRODUCTION}

The U.S. particle physics program focuses on important measurements that are done primarily at accelerator laboratories, supplemented by others that are not based at accelerators. Historically, we have found that a balanced approach of high-energy, high intensity and high precision experiments offers the best prospect for answering the compelling questions proposed in Chapter III.

Accelerator-based experiments use high energies and intensities to make direct and incisive tests of the Standard Model and to search for new physics that lies beyond. The program is centered on four laboratories, the Fermi National Accelerator Laboratory (Fermilab), the Stanford Linear Accelerator Center (SLAC), Brookhaven National Laboratory (BNL), and the Laboratory of Nuclear Studies at Cornell. This work is complemented by U.S. physicists collaborating on experiments abroad, and by the large number of foreign collaborators who contribute substantially to the work in this country.

The mainstay of the U.S. particle physics program is the physics community based at universities and laboratories. The university groups play a special role because they are in the closest contact with students, at the graduate and undergraduate levels, and train future leaders of science and technology. Indeed, the people who leave our field and apply our technologies and concepts to the world at large are among our most important contributions to society.

During the past twenty years, the Standard Model has become the paradigm for particle physics, and has been tested to higher and higher precision. It is so tightly woven and so powerfully predictive that one measurement affects many others. For example, the mass of the top quark is related to the level of charge parity $(\mathrm{CP})$ violation in kaon decays; rare $B$ meson decays give us hints about the nature of the Higgs particle; and neutrino oscillation experiments can provide clues to the large-scale structure of the universe. This inter-connectedness guides us in our choice of experiments and allows us to extrapolate beyond what we can measure directly.

Because of the long time scale of high-energy physics experiments, we can sketch the broad outline of the U.S. domestic program well into the next decade. In the near term, we can anticipate a strengthening of the evidence for the top quark at Fermilab, and more importantly, the 
first direct measurements of its properties. The Tevatron collider and Main Injector will also permit precise measurements of the $W$ mass and allow a general exploration at the highest energy frontier currently accessible with accelerators. Fixed-target experiments at Fermilab and Brookhaven will use high-precision measurements and high-intensity kaon beams to probe $\mathrm{CP}$ violation and other new physics.

At SLAC, studies of the $\mathrm{Z}$ boson with polarized electron beams will allow experiments to make precise determination of the weak mixing angle. The combination of this result with complementary studies of the $\mathrm{Z}$ made at LEP with unpolarized electrons and precision measurements of the $W$ and top masses at Fermilab will not only constrain the Higgs structure, but will begin to open windows to possible new physics.

The CLEO experiment at the Cornell Electron Storage Ring (CESR) has been our most prolific source of information on B decays. More recently, experiments at the Fermilab Tevatron collider have begun to contribute. These two laboratories will be rich sources of $B$ physics for the decade to come. Indeed, the differences in their experimental environments lead to complementary strengths. It is not inconceivable that one of these experiments will make the first observation of $\mathrm{CP}$ violation in $B$ meson decays. However, the most comprehensive study of CP violation is expected to be conducted at the SLAC B-factory. With its combination of unequal beam energies, high luminosity and clean environment, it will provide a breadth of measurements that will thoroughly test our understanding of $\mathrm{CP}$ violation. In the longer term, the experiments at the Fermilab collider may well produce precise measurements of $\mathrm{CP}$ asymmetries in a limited number of decay modes if the technical difficulties of isolating these decays in the complex environment of a high-luminosity hadron collider can be overcome.

As is clear from this discussion, our present accelerators and our approved construction programs represent a substantial investment with significant discovery potential. The present domestic facilities and their upgrades will be major components of the U.S. research program for the next decade. On the longer time scale, they offer opportunities for innovation well beyond the presently planned experiments.

In the wake of the cancellation of the Superconducting Super Collider (SSC), many in the U.S. community are just beginning to think about how to best utilize and upgrade the existing domestic facilities to continue research on the frontiers of energy, intensity and precision. 
Therefore, it is important to emphasize that our vision must include strong support for excellent future initiatives, including the innovative use of our national laboratories beyond what is presently seen.

We describe below in more detail the research programs at the accelerator laboratories in the United Sates and overseas.

\section{B. FERMILAB}

The Fermilab Tevatron Collider is the highest energy accelerator in the world and is providing some of the most exciting results in the field. With the first evidence for the top quark now in its grasp, a new era of exploration will begin. Because top is so massive, it plays an essential role in the electroweak theory. For instance, an important test of the Higgs mechanism is possible once the value of the top quark mass is known accurately and is combined with a precise measurement of the $W$ mass.

The Main Injector Project, the largest of several accelerator upgrades, is scheduled for completion in January 1999. It will replace the original Main Ring, which now functions as an acceleration stage feeding the Tevatron, and it will provide several benefits. First, the larger number of protons and antiprotons available for collisions will give a five-fold increase in the event yield for the collider experiments. Second, the Main Injector allows for intense $120 \mathrm{GeV}$ beams, well matched to kaon and neutrino experiments, test beams for collider experiments, and conceivably test beams for Large Hadron Collider (LHC) experiments. In addition, these beams will now be available during collider operations, something which is impossible with the present injection system.

a. Colliding Beam Experiments. There are two large detector groups at the Tevatron Collider-the Collider Detector at Fermilab (CDF) and D-Zero collaborations. Both detectors are presently being upgraded to take advantage of the increased luminosities. Each collaboration consists of over 400 physicists from institutions in North and South America, Asia, and Europe. Foremost among the physics topics being pursued is the top quark. Detailed studies of its properties, decay modes, and interactions with other particles will reveal much about the nature of this unique particle and require substantial numbers of events in many different decay modes. (By way of analogy, the $b$ quark was discovered in 1977 and an exploration of its properties not only is continuing, but is the subject of new facilities now under construction.) If top is indeed near $170 \mathrm{GeV}$, as recent 
evidence suggests, then the Main Injector/Tevatron complex will provide sufficient beam intensities to make a good first exploration of its properties. Indeed, until the start of the LHC, Fermilab will be the only place in the world where top can be studied.

The Tevatron Collider also produces large numbers of $W$ bosons. A primary goal of CDF and D-Zero is to make a highly accurate measurement of the $W$ mass. The $W$ mass is one of the most fundamental parameters in the Standard Model and, like the top mass, it is more than another number in a table of elementary particles. To shed light on the mass and nature of the Higgs boson, there is a threshold of precision needed: approximately 50 $\mathrm{MeV}$ for the $W$ mass and $5 \mathrm{GeV}$ for the top mass. Presently the $W$ mass is known to roughly $200 \mathrm{MeV}$. With the large event yield at the Tevatron when the Main Injector is completed, one can approach the needed level of precision for both the top and the $W$ masses.

The Tevatron, since it operates at the energy frontier, also has the capability to uncover new massive particles. Searches for supersymmetric particles and new gauge bosons, and departures from the point-like nature of quarks are the purview of the Tevatron collider until the LHC turns on.

The CDF detector has already demonstrated the tremendous potential of hadron machines for exploring the physics of $B$ mesons. Although studies of the $b$ quark are perhaps less newsworthy than evidence for top, discoveries of new particles containing the $b$ quark are occurring all the time, such as the recent discovery of the $B_{\mathrm{s}}$, both at the Large Electron Positron (LEP) and CDF. Collider experiments are able to explore the full range of predicted bound states containing the $b$ quark and, because of the high b-cross section at high energy, to search also for rare decay modes. Some of these rare decay modes are highly suppressed in the Standard Model and provide sensitive probes for new physics. The high intensity of the Main Injector may also provide the opportunity to study $C P$ violation in a limited, but significant, number of $B$ decay modes which are particularly easy to isolate in the complex environment of a hadron collider. 
b. Fermilab Fixed-Target Program. Fermilab has an extensive series of beam lines that are used to supply fixed-target experiments with beams extracted from the Tevatron. These lines derive secondary beams from a primary proton beam of $800 \mathrm{GeV}$-the highest energy available in the world. Proton, pion, charged and neutral hyperons, neutral kaon, muon, photon, and neutrino beams are all available. The most recent run of the fixed-target program resulted in a wide range of interesting results. These included studies of the origin of $\mathrm{CP}$ violation in the decay of the neutral $K$ meson, important new measurements of the properties of particles containing charm and strange quarks, and measurements of the structure of nucleons and nuclei. Interesting results in the bound states of charm quarks were obtained from an experiment using antiprotons.

The upcoming run of the fixed-target program, before the shutdown for Main Injector installation, will include an experiment $(\mathrm{KTeV})$ that aims to achieve a level of precision for $\mathrm{CP}$ violation with $K$ mesons in the parameter $\varepsilon^{\prime} / \varepsilon$ which will probe the standard model of $\mathrm{CP}$ violation. This run will also include a new neutrino experiment which will examine the structure of the proton and measure the weak mixing angle to high precision, complementing the result obtained by several different experiments (e.g., atomic parity violation experiments, $Z$ decays, etc.) and will further test the electroweak theory. There will also be a very high statistics study of charm decays and additional studies of the bound states of charm quarks.

With the Main Injector, the proton intensities available from the Tevatron at $800 \mathrm{GeV}$ will double. Furthermore, the Main Injector can directly supply a series of fixed-target experiments with high intensity beams derived from $120 \mathrm{GeV}$ protons. The intensity of the Main Injector beams will result in a factor of 10 improvement in the yield of kaons, resulting in significantly improved reach for rare kaon decays and $\mathrm{CP}$ violation and will allow a very high statistics exploration of charm decays. The Main Injector can also provide the beams needed for a significant set of experiments to search for neutrino oscillations-both with substantially higher rates, and also with the capability of performing long baseline experiments. Both the kaon and neutrino capabilities at Fermilab offer interesting opportunities for probing physics beyond the Standard Model. 
c. Accelerator Research and Development. A variety of research and development on accelerators is being done at Fermilab. Ongoing research and development is directed at increasing operating efficiency, increasing luminosity of the Tevatron, and supporting construction of the Main Injector. Advanced research and development includes a study designed to increase the luminosity of the Tevatron by applying techniques used to store antiprotons at lower energies. Through its expertise in cryogenic and $L$ band radiofrequency technology, Fermilab is also making important contributions to the TESLA international collaboration on linear collider research and development.

d. Further Collider Upgrades. There are a number of important physics topics that could be explored with further upgrades to the collider and there has been considerable activity at Fermilab to begin understanding these possibilities. Collider upgrades could proceed along the paths of enhancing the energy, the luminosity, or both. More detailed studies of the top quark and $B$ mesons would be possible with both energy or luminosity upgrades. An increase in the beam energy by a factor of two would permit an exploration of a substantial range of likely mass states of supersymmetric particles.

A clear understanding of the potential physics (both collider and fixed-target) that could be achieved with these possible Tevatron improvements is needed. How that physics might compare to, or complement, the potential program of the LHC, and give a longterm base for hadron physics in the U.S. past the start of the LHC must be investigated. Both cost and operating down time must be weighed against any potential performance gains. Fermilab should be encouraged in their studies of future ideas, and when a mature proposal is put forward, it should be evaluated in the context of the overall world program.

\section{STANFORD LINEAR ACCELERATOR CENTER}

The Stanford Linear Accelerator Center is the highest energy electron-positron facility in the United States, with a diverse program of ongoing research, upgrades to existing facilities and accelerator research and development. The current experimental program exploits SLAC's unique ability to produce highly polarized electron beams up to $50 \mathrm{GeV}$ in energy. The near-term program focusses on studies of the $\mathrm{Z}$ boson and of the nucleon spin structure. Smaller experiments are also supported. An example is an experiment in 
progress to probe Quantum Electrodynamics at very high fields by colliding an intense laser beam with the high-energy electron beam. In addition, U.S. participation in the Bejing Electron Storage Ring is centered at SLAC. These ongoing efforts will provide a full program of research opportunities for the next four to five years while SLAC prepares to explore $\mathrm{CP}$ violation with the asymmetric-energy $B$-factory (PEP-II).

a. SLAC Linear Collider Program. The luminosity of the Stanford Linear Collider (SLC) has grown steadily, and in 1993 the Stanford Large Detector (SLD) detector recorded $50,000 \mathrm{Z}$ decays produced with a highly polarized electron beam. These data have given the world's most precise single measurement of the weak mixing angle. The SLD detector will continue to accumulate data until operation of the $B$-factory begins. At that point, the data sample should consist of about a half million $\mathbf{Z}$ decays, and will yield a measurement of the $Z$ polarization asymmetry that will provide an important and stringent test of the Standard Model.

In addition to polarized electrons, the SLD experiment has another unique advantage in the study of $Z$ decays: a vertex detector, based on charged-coupled devices, providing truly three-dimensional information on the location of charged particles very close to the micron-sized beam. This detector is currently being upgraded. The combination of polarized electrons and the upgraded three-dimensional vertex detector will allow the SLD collaboration to make significant measurements involving polarized heavy quarks and leptons such as b quarks and tau leptons. These studies are sensitive to the presence of more massive particles, such as the top quark and the Higgs boson, and should provide further precision tests of the electroweak theory.

b. Fixed-Target Program. Fixed-target electron-nucleon scattering experiments will continue at SLAC in 1996, after an upgrade of the beam line to transport $50 \mathrm{GeV}$ polarized electron beams into the target area. Experiments using polarized targets will extend measurements of the distribution of quark and gluon spins inside the neutron and proton, providing important tests of quantum chromodynamics (QCD). Beyond these measurements, the fixedtarget user community, comprised of about 100 high-energy and nuclear physicists, is exploring the possibility of further experimentation with the high-energy polarized beam. 
c. Asymmetric-energy $B$-factory. At the $B$-factory, mesons containing the bottom quark will be produced with unequalenergy electron and positron beams, leading to B mesons which are moving in the laboratory. This allows measurement of the difference between decay times of the two B mesons. CP violation would be revealed for certain $B$ decays as asymmetries in these time-difference distributions for $B$ and $\bar{B}$ mesons. These asymmetries are theoretically interpretable in terms of fundamental parameters of the Standard Model and will provide thorough tests of our present view of $\mathrm{CP}$ violation resulting from quark mixing. The highly-constrained events produced in electron-positron collisions are the ideal environment for reconstructing many different final states. By measuring asymmetries in a variety of decay modes, the Standard Model is not only constrained but tested for consistency. Clues to the source of any measured inconsistencies can again come from comparing asymmetries in many decay modes.

The SLAC B-factory design, based on the existing Positron Electron Project (PEP) storage ring, is the culmination of an accelerator research and development program carried out by SLAC, Lawrence Berkeley Laboratory (LBL) and Lawrence Livermore National Laboratory (LLNL). The construction of the $B$-factory began in FY 1994, with initial operation expected in 1998. The detector collaboration for the B-factory is currently being formed with wide international participation. A large number of institutions have expressed interest in collaborating on this experiment, including groups from the U.S., Canada, U.K., France, Italy, and Germany.

d. Linear Collider research and development. Concurrent with the operation and development of SLC, SLAC supports an advanced accelerator research and development program for high-energy electron-positron linear colliders. This program is part of the worldwide collaboration on research and development for a nextgeneration high-energy electron-positron collider, and includes close ties with other laboratories in the U.S., Europe, Japan, and Russia. With the only operating linear collider in the world, SLAC's role in this endeavor is vital. 
A prototype beam line has been designed and built at SLAC to address the problem of the spot size of a linear collider at the collision point. The international Final Focus Test Beam Collaboration has initiated this year a vigorous experimental program in this area.

A program to develop the high power microwave sources and acceleration systems (necessary for a future collider) is under way, and a prototype $\mathrm{GeV}$-scale test accelerator is being constructed.

e. Stanford Synchrotron Radiation Laboratory. The Stanford Synchrotron Radiation Laboratory (SSRL) operates the SPEAR storage ring to serve a large community of university and industrial researchers in the physical and biological sciences. A new dedicated injector for SPEAR has led to substantial increases in beam usage in recent years. The SSRL is a division of SLAC.

\section{BROOKHAVEN NATIONAL LABORATORY}

The Alternating Gradient Synchrotron (AGS) at BNL, even though in operation for over thirty years, continues to offer opportunities for exploration of forefront issues in high-energy physics. The AGS is a proton synchrotron which normally provides $24 \mathrm{GeV}$ protons to fixed-target experiments. The recently completed AGS Booster provides an increase in flux well beyond what was available only a few years ago. This makes the AGS by far the most intense source for secondary beams and well suited for experiments that depend on high fluxes, such as rare kaon decay, high precision experiments and neutrino studies. Indeed, to date the best sensitivity for rare kaon decays has been obtained at the AGS.

a. Rare Kaon Decays. Rare kaon decay experiments are a major part of the ongoing AGS physics program. They divide naturally into two general sub-areas: (1) decays forbidden in the Standard Model, but which could occur in various possible extensions signaling new physics at very high mass scales, and (2) highly suppressed standard model decays which are sensitive to effects hidden from view in most other particle decays, such as heavy quark mixing and top mass effects. Because these decays are rare, they are also potentially sensitive to new physics. 
The first category includes searches for kaon decays which violate the law of lepton generation number conservation. Experiments that will begin data-taking at the AGS this year and continue for several years, will focus on these modes and should ultimately be capable of detecting decays as rare as one in a trillion decays. At this level of sensitivity, the mass scale probed for new physics extends up to about $200 \mathrm{TeV}$--much above what can be reached directly by accelerators in the foreseeable future.

The second category includes decays which provide novel measurements of the underlying parameters of the Standard Model, and also have sensitivity for new physics if the decay occurs with a branching fraction above the Standard Model level. An example is the decay $\mathrm{K}^{+} \rightarrow \pi^{+} v \bar{v}$ (expected at the level of one part in ten billion), which can measure the coupling between the top and down quarks. An experiment with this focus is under way at the AGS and should observe this mode in the next two to three years, if its rate is in accord with our present expectations.

b. Other Experiments. The AGS physics program is diverse and extends beyond the rare kaon decay experiments discussed above. An experiment to measure the anomalous magnetic moment of the muon will provide a factor of 20 improvement over the previous best measurement. As with other precision electroweak measurement, this is a possible probe of new effects occurring at high mass scales. This level of precision addresses for the first time the contribution of the weak interactions and probes new physics up to $5 \mathrm{TeV}$.

A series of experiments also focuses on QCD physics. Examples include experiments searching for exotic quark-gluon states, color transparency, and six-quark states.

The relatively low energy of the AGS combined with the high proton flux also make it a promising source of neutrinos for a future long-baseline oscillation experiment. Such an experiment has been approved by the laboratory management, but it needs to be evaluated in the context of other possible neutrino oscillation experiments.

c. Relativistic Heavy Ion Collider. The AGS is also used to accelerate heavy ions to $10-15 \mathrm{GeV}$ per nucleon as part of the Department of Energy's Nuclear Physics program. When the Relativistic Heavy Ion Collider (RHIC) is completed at BNL, the 
AGS will serve as the injector and a major program of experiments probing QCD dynamics at high density and temperatures will be initiated. Polarized protons at RHIC will also offer unique opportunities for QCD studies and nucleon polarized structure function measurements.

In the RHIC era, opportunities to conduct high-energy physics experiments, such as rare kaon decay experiments and neutrino oscillations, continue to exist at the incremental cost of running the facility to support these experiments.

d. Accelerator Research and Development. Accelerator research and development at BNL is directed at the AGS Booster upgrade for intensity improvement and preparation for heavy ion injection into RHIC, including superconducting magnet development. BNL also supports a significant facility for research and development on advanced accelerator concepts. The facility includes highpowered lasers, and an intense electron source. Three beam lines with a fourth soon to be added provide users with laboratory space for experiments employing one or more of these beams simultaneously. A number of speculative new acceleration concepts are now being studied, including the possibility of laser driven accelerators with high electric field.

\section{E. CORNEll Laboratory OF NUClEAR StUdies}

a. CESR Program. The CLEO experiment at the Cornell Electron Storage Ring (CESR) is an extremely productive source of physics results on the charm and bottom quarks and the tau lepton. With its current sample of millions of $B$ mesons and its excellent detector performance, the CLEO experiment has yielded extremely precise studies of decays of the bottom quark. For example, it has uncovered the first evidence of $b \rightarrow s \gamma$ transitions-a special kind of rare decay process that is highly sensitive to effects outside the Standard Model. Furthermore, the most precise measurement of the coupling between the bottom and up quarks, a parameter crucial for setting the scale of $\mathrm{CP}$ violating phenomena in the $B$ system, has come from CESR.

A two-stage upgrade program is now under way to raise the luminosity of CESR and to improve the capabilities of the CLEO detector. The current luminosity of CESR produces about $1.5 \mathrm{fb}^{-1}$ per year $\left(f b^{-1}\right.$ denotes $10^{39}$ per square centimeter.) In the first phase, to be completed in early 1995, the luminosity is being 
increased by about a factor of three above its current level, and a three-layer silicon vertex detector is being installed in the detector. At the completion of the second phase, scheduled for 1998 , the luminosity is expected to be increased by a further factor of two and the detector will be equipped with a new drift chamber and an improved particle identification system. While CESR is primarily supported by the National Science Foundation, 13 of the 21 collaborating CLEO universities are supported by Department of Energy.

The goal of the CESR upgrade program is to achieve luminosities in excess of $10 \mathrm{fb}^{-1}$ per year late in this decade. For at least the next five years, CLEO will have a monopoly on $b$ physics conducted at an electron-positron machine at the $b$ threshold. In addition it will have the largest samples of tau and charm decays in the world. With the ongoing accelerator improvements and enhancements to the detector, CLEO can expect significant increases in sensitivity to important physics parameters and for the rates of rare and forbidden $B$ decays.

b. Accelerator Research and Development. CESR is currently the highest luminosity electron-positron collider in the world at any energy, and the laboratory is a major center for research in accelerator physics. Many important results in accelerator physics have been acquired during the evolution of this machine, and new techniques, such as the use of non-zero collision angles, are expected to be developed as part of the upgrade program.

Cornell also supports significant work on the development of superconducting linear electron accelerators. As participants in the TESLA linear collider research and development program, Cornell has a major responsibility for the superconducting cavity gradient improvement program.

In addition to the particle physics program, CESR provides the world's highest intensity $\mathrm{x}$-ray beams to a large user community in the physical and biological sciences. These beams are also used for engineering studies of accelerator and detector elements. 


\section{F. FOREIGN LABORATORIES}

American physicists actively participate in research activities at five major laboratories overseas. The opportunities available at these facilities are largely unique, and U.S. participation illustrates the strong international flavor of research carried out in high-energy physics.

a. DESY. The U.S. high-energy physics community has a substantial presence at the Deutsches Elektronen Synchrotron accelerator (DESY) in Hamburg, Germany. Eleven U.S. institutions, with about sixty Ph.D. physicists, are working in its two large detectors, ZEUS and $\mathrm{H1}$, at the high-energy electron-proton collider Hadron-Elektronen-Ring-Anlage (HERA). In addition there are a number of U.S. groups-some supported by the Division of Nuclear Physics - engaged in an upcoming study of polarized ep scattering at lower energies, with the aim of elucidating the spin structure of the proton and neutron.

The physics program at HERA is of considerable interest, extending the study of deep inelastic scattering to momentum transfers of the order of the electroweak boson masses and probing the structure of the proton in unexplored regions. These latter measurements, in particular, will probe QCD in a region of great theoretical interest, where new phenomena such as the shadowing and saturation of quark and gluon interactions may become apparent. Furthermore, knowledge of the proton structure function in the HERA regime will be crucial for predicting and understanding data from hadron colliders in the $\mathrm{TeV}$ region. Studies are also now in progress for a fixed-target experiment dedicated to the study of $B$ mesons and $C P$ violation in system using the proton beam halo available at HERA.

DESY is also involved in linear collider research and development based both on normal and superconducting technology and is part of the international collaboration aimed at construction of an electron-positron linear collider. DESY is the leader of the TESLA collaboration on superconducting linear collider technology.

b. KEK. At KEK in Japan, the current $30 \mathrm{GeV}$ on $30 \mathrm{GeV}$ electron positron collider (TRISTAN) is being converted into an asymmetric $B$-factory (TRISTAN II) to study the origin of CP violation in the $B$ sector. This accelerator has lower energy but much higher luminosity than the original TRISTAN project and 
has many similarities in both objectives and machine design to the $B$-factory at SLAC. Two new rings, of 3.5 and $8.0 \mathrm{GeV}$, will be installed in the current TRISTAN tunnel.

Because of its higher luminosity goal (a factor of three beyond that of the SLAC B-factory) and the limitations of the existing injector, the KEK design employs some aggressive new technologies. When its design intensity is achieved, it will complement, and provide healthy competition for, the $B$-factory at SLAC in addressing a most fundamental problem. Recently an international group, BELLE, has been formed to build the detector for doing experiments at TRISTAN II. A relatively small U.S. contingent is likely to join this group.

Kaon decay experiments are carried out at the $12 \mathrm{GeV}$ proton synchrotron. Again, this program provides healthy competition for rare $\mathrm{K}$ decay experiments at BNL and Fermilab. This facility is a valuable tool not only for Japanese physicists, but also for Asian colleagues, as well as U.S. physicists to develop detectors and prepare experiments like Superkamiokande.

Accelerator physicists at KEK are actively pursuing the technology of electron-positron linear colliders. Work at the laboratory centers on the advancement of room temperature accelerators. KEK participates in both the U.S.-Japan Collaboration on future accelerators and the Collaboration on research and development towards $\mathrm{TeV}$ scale electron-positron colliders.

c. CERN. The European Laboratory for Particle Physics (CERN) has a rich variety of experimental initiatives in high-energy physics. These include:

i. LEP and LEP II: The LEP collider at CERN provides high-energy electron positron collisions to four large multipurpose experiments, ALEPH, DELPHI, OPAL, and L3. These experiments are run by collaborations of 300 to 500 physicists from around the world. A total of roughly 250 U.S. physicists participate in these experiments, playing a leading role in L3, with a strong presence on ALEPH and OPAL. Since the start of the project, the LEP detectors have each recorded the results of two million $Z$ boson decays, allowing extremely precise measurements of many critical Standard Model parameters. Among these are the mass of the $Z$ and the rate of its decay. This latter information, also determined 
at the SLC, can be used to infer that there are only three generations of quarks and leptons-a fundamental result. The precise information from LEP, in the context of the Standard Model, serves to constrain the mass of the top quark. If subsequent measurements confirm the evidence of a top quark of a mass of $174 \mathrm{GeV}$, as indicated by recent results from Fermilab, the remarkable agreement with the mass value inferred from LEP data $(177 \mathrm{GeV})$ is an impressive success of the Standard Model. In addition to measurements associated with the standard model properties of the $Z$ boson, LEP is providing important new information on the properties of the $b$ quark.

The future program for the LEP machine at CERN includes an ambitious upgrade using super-conductive cavities to achieve higher machine energies, slightly above the energy threshold for producing $W$ boson pairs. It is expected that this new physics program will start in 1996, followed by three years (1997-1999) of data accumulation. Some of the main physics goals include a high precision measurement of the $W$ mass to a level of accuracy comparable to that which is aimed for at the Fermilab Main Injector. This upgrade should allow the LEP experiments to search for a Higgs boson up to a mass of $90 \mathrm{GeV}$ and for supersymmetric particles in roughly the same range. These exploratory windows are complementary to the physics opportunities at Fermilab and are of considerable interest.

ii. Fixed-Target Experiments: At CERN there is a wide variety of fixed-target experiments. In particular, CERN is also engaged in a high precision $\mathrm{CP}$ violation experiment with the same reach as the $\mathrm{KTeV}$ experiment at Fermilab. U.S. physicists participate in the NOMAD neutrino oscillation experiment aimed at studying the rate of muon neutrinos changing into tau neutrinos. Other U.S. physicists participate in an experiment which studies the spin content of the proton which is similar in scope to an experiment currently being done at SLAC. Finally, there is also a substantial U.S. participation in heavy ion fixed-target experiments.

iii. LEAR: The CERN low energy antiproton ring (LEAR) is a unique accelerator where studies of low energy spectroscopy, as well as the antiproton mass and properties, are carried out. United States physicists participate in a number of these experiments, but the facility is likely to be shut down later on in the decade. 
iv. CLIC: CERN accelerator scientists are participating in the international effort to develop viable technology for electronpositron linear colliders, known as CLIC. The CERN approach exploits the two-beam accelerator idea operating at very short wave length.

d. Beijing Electron Storage Ring. The Beijing Electron Storage Ring is designed to study the tau/charm energy range with approximately five times the luminosity of SPEAR. Since late 1990, U.S. physicists from several institutions have been active in this program. The BESR collaboration has measured the mass of the tau lepton with a six-fold improvement in precision. The goal of the current program is to further study the D mesons and other bound states of charm quarks.

e. Frascati. A high luminosity Phi Factory is being built in Frascati, Italy aimed at studying $\mathrm{CP}$ and $\mathrm{CPT}$ violation phenomena in the Kaon system. A small U.S. contingent is involved in the KLOE detector presently under construction. 


\section{THE ENERGY FRONTIER}

\section{A. INTRODUCTION}

The advent of colliding-beam accelerators over two decades ago redefined the study of elementary particle physics at the highest energies. Since that time, particle colliders have been the instruments used by physicists to explore the energy frontier. Discoveries and advances on this frontier have been crucial to the development of the remarkable theoretical description of nature we call the Standard Model.

Despite the Standard Model's ability to describe all that we see at present-day energies, the crucial issue of electroweak symmetry breaking must be resolved by experiments at TeV-scale colliders. Such investigations promise a deep and clear understanding of the origins of mass. It is this prospect that excites and motivates us to explore further along the energy frontier.

The Tevatron at the Fermi National Accelerator Laboratory (Fermilab) is presently the highest energy accelerator in the world. The Large Hadron Collider (LHC), planned to begin operation at the European Laboratory for Particle Physics (CERN), circa 2003, will be a direct descendant of the Tevatron. The LHC will be a proton-proton collider with seven times the energy and up to 100 times the luminosity of the Tevatron. It will open new windows for discovery and present important opportunities for confronting some of the fundamental questions posed in Chapter III.

The LHC is a high-performance collider designed to operate at 14 $\mathrm{TeV}$ of energy with luminosity of $10^{34} \mathrm{~cm}^{-2} \mathrm{sec}^{-1}$. In June of this year, CERN will seek approval from its member states for the LHC project. CERN desires inter-regional collaboration in the design and construction of the accelerator and detectors. The project is still in its design stage, so this would be an appropriate time for initial U.S. participation.

Successful construction and operation of the Tevatron, as well as investment in research and development for the Superconducting Super Collider (SSC), have resulted in a core of U.S. scientists and engineers with the world-class experience and knowledge needed for the design, implementation, and operation of superconducting accelerators. These people are an invaluable resource that would allow the U.S. to make important contributions to the LHC and to sustain the experience and knowledge required to address the challenges of future hadron accelerators. 
Expertise gained by U.S. physicists from work at the Tevatron and in preparation for the SSC would be valuable not only for the completion of the machine but also for its detectors. The detectors required for the LHC pose many challenges for the scientific community. Research and development in both the U.S. and Europe have capitalized on rapid change in electronics technologies to create particle detectors that promise to meet the stringent demands of the LHC environment.

The LHC will be a great step on the energy frontier, but it will not be the last step. Compelling questions surely lie beyond the physics reach of the LHC. Participation by the U.S. in the LHC would further strengthen our position among world leaders in the development of strategies and mechanisms needed for global cooperation on largescience projects. This would enhance U.S. capabilities to host such projects, including those of high-energy physics.

The technology of the LHC does not exhaust the possibilities for proton storage rings. Preliminary examination indicates that it may be technically feasible to build a proton collider with beam energies up to ten times those of the LHC with technology that could be developed during the next decade. For the U.S. to maintain its place among the leaders of the world high-energy physics community, it will be important to participate in regional or global collaborations to carry out the research and development required for such a future machine.

$\mathrm{A} \mathrm{TeV}$-scale electron-positron collider offers unique opportunities to extend and complement experiments done at the LHC. Studies of physics goals for such a collider have progressed in a series of international workshops. A consensus has been forged that the next electron-positron collider should have an initial center-of-mass energy of approximately $500 \mathrm{GeV}$ and luminosity in excess of $10^{33} \mathrm{~cm}^{-2} \mathrm{sec}^{-1}$, and be eventually capable of reaching to $1 \mathrm{TeV}$ and beyond with luminosity in excess of $10^{34} \mathrm{~cm}^{-2} \mathrm{sec}^{-1}$.

A world-wide effort is under way to develop the understanding of accelerator physics and the technology needed to build the nextgeneration high-energy electron-positron collider. Prototype accelerators now under construction will provide answers to questions of optimization and cost. This international research and development program is focused on formulating conceptual designs based on proven technology with reliable cost estimates in the second half of the present decade. 
For the longer term, it is important to investigate new acceleration systems, as well as techniques for accelerating particles other than protons and electrons. Many novel ideas are being discussed, but much work remains to be done to develop them into practical vehicles for accelerator-based high-energy physics.

\section{B. THE LHC MACHINE}

If approved by the CERN Council, the LHC will define the world energy frontier in high-energy physics beginning approximately ten years from now. The LHC is designed to be housed in the present Large Electron Positron (LEP) tunnel, with a center-of-mass energy of $14 \mathrm{TeV}$ and peak luminosity of $10^{34} \mathrm{~cm}^{-2} \mathrm{sec}^{-1}$ for proton-proton collisions. Operations with heavy ions are also foreseen.

The superconducting magnets of the LHC are intended to operate at 8.7 Tesla and a temperature of $1.8 \mathrm{~K}$. The corresponding parameters for the SSC were to have been $40 \mathrm{TeV}, 10^{33} \mathrm{~cm}^{-2} \mathrm{sec}^{-1}$ with 6.6 Tesla magnets operating at about $4 \mathrm{~K}$. The beam currents and magnetic fields required to achieve the full LHC design goals are more challenging than for the SSC and leave less potential for further upgrade. While initial operation of the LHC at $10^{33} \mathrm{~cm}^{-2} \mathrm{sec}^{-1}$ should be relatively straight forward, pushing on to $10^{34} \mathrm{~cm}^{-2} \mathrm{sec}^{-1}$ will stress beam stability control, synchrotron radiation heat removal from the cryogenic beam chamber and radiation tolerance of the detectors and immediately adjacent accelerator components. Considerable engineering development remains. Nevertheless, these goals appear technically feasible.

Superconducting magnet development for the main dipoles is a critical element of the LHC program. European industry is heavily involved in this work, in conjunction with a growing CERN magnet development and test capability. Magnet specifications have recently been changed to improve manufactureability and reliability, and work on a new design is under way.

United States expertise gained in the planning and development of SSC magnets and other technical components has the potential to help minimize the duration of the construction schedule. A joint CERN-U.S. workshop recently identified a number of areas where this expertise can be brought to bear with advantage. They include magnet modeling and materials specification activities and tests, together with some vacuum components and accelerator physics computations. In the longer run, the U.S. could also provide considerable help in construction of such major subsystems as injection lines, insertion magnets, ring quadrupoles, and 
beam tube liners. Collaboration in LHC construction would be rewarding to U.S. physicists and engineers, and would also yield experience and knowledge with which to address the problems of future hadron accelerators.

The CERN management's current schedule foresees completion of construction in 2002. This schedule envisions three years of magnet development, two years of magnet pre-production, and three more years to complete production. As planning of technical work and financing becomes more detailed, the schedule may be extended by one or two years.

\section{THE LHC EXPERIMENTAL PROGRAM}

The presently proposed LHC experimental program will allow the world-wide physics community to address important areas of interest. In addition to the two general purpose detectors, ATLAS and CMS, a large heavy-ion detector collaboration, ALICE, has also been approved. A dedicated $b$-physics detector will be constructed, and three Letters of Intent for this detector have been submitted. All four detectors are expected to be components of the initial LHC physics program, and American institutions have expressed interest in participating in each of them.

The estimated cost of each general-purpose detector, according to CERN accounting practices, is roughly $400 \mathrm{MSF} .^{1}$ Detailed Technical Proposals are due in December 1994, including a relatively comprehensive preliminary division of responsibilities. During 1995, it is anticipated that formal agreements (and funding commitments) will proceed towards their final form. An eight-year project schedule (1995-2002) is anticipated, with construction beginning in 1997, and with first physics runs in the year 2003. Details of the construction schedule, and the corresponding funding profile, are just now being studied.

1. High Luminosity Issues. To fully exploit the physics capabilities of the LHC, it is important for the general-purpose detectors to be capable of operation at the full design luminosity of $10^{34} \mathrm{~cm}^{-2} \mathrm{sec}^{-1}$ (see the following discussion of LHC physics). A typical general purpose detector for a high-energy hadron collider consists of an inner tracking volume,

${ }^{1}$ The cost of an experiment in CERN accounting includes only the cost of materials and external labor. 
followed by a massive calorimetry system, surrounded by a muon spectrometer. The high design luminosity of LHC poses severe challenges to all three of these major detector components.

At a luminosity of $10^{34} \mathrm{~cm}^{-2} \mathrm{sec}^{-1}$, the LHC detectors must reconstruct rare and interesting events in the midst of 15-20 interactions every 25 nanoseconds, each producing roughly 40 charged tracks within the detector acceptance. This high fluence of particles causes radiation damage in the detector elements and their readout electronics.

In addressing this environment, several factors are critical. Very fine segmentation of the detectors is needed to avoid confusion in reconstructing events. A typical LHC detector has a tracking system consisting of $10^{7}$ channels, a calorimeter system with $10^{5}$ towers, and a muon system containing $10^{6}$ channels. Extraordinary progress in microelectronics design and production, as well as in other relevant detector technologies, now allows such systems to be implemented at a manageable cost.

The 25-nanosecond bunch-crossing interval places severe constraints on the speed of the individual detector elements and requires novel designs. Furthermore, the high radiation environment implies that the detectors and their electronics must be radiation hardened to survive exposure to these conditions. The challenges are particularly acute in the inner regions of the tracking volume and the forward regions of the calorimetry, and replacement or refurbishment of the detector elements in these areas may be required over periods of several years.

During the previous five years, the SSC and LHC communities have engaged in an extensive program of detector research and development and have addressed these issues for the major detector components, as well as for readout electronics and triggering. Enormous technical progress has been made in many areas, and serves as the basis for the present detector designs.

The collaborations themselves have adopted the ambitious goal of detector survival for a period of ten years at $10^{34} \mathrm{~cm}^{-2} \mathrm{sec}^{-1}$. While it is still too soon to be sure that this goal can be achieved in all cases, there is good reason to be optimistic. Furthermore, the complementarity of the two general-purpose detectors enhances confidence in the overall program. However, to clarify the physics capabilities of the LHC in the presence of the challenge of reaching full luminosity, a physics program at a luminosity of $10^{33} \mathrm{~cm}^{-2} \mathrm{sec}^{-1}$ will also be discussed. 
2. The General-Purpose Detectors. The ATLAS and CMS detectors are both being designed to carry out a broad range of physics studies at luminosities up to the maximum design luminosity of the LHC. In the following, the overall architecture of the two detectors is compared and contrasted, emphasizing the complementary approaches taken in addressing the LHC physics challenges.

Both detectors rely on large magnetic field volumes created by superconducting magnets to measure the momentum of energetic charged particles. The ATLAS detector makes its initial measurements in a modest-sized solenoidal field of 2 Tesla surrounding the inner tracking volume. The muon spectrometer then uses a second magnet with a toroidal field to provide high-precision measurements. The CMS detector uses a single large, high-field (4 Tesla) solenoid, surrounding the tracking volume and the calorimetry, to provide the measurements for both the inner tracking volume and the muon spectrometer.

The smaller ATLAS inner tracking system uses a mixture of discrete high-precision measurements and lower-precision continuous measurements to provide the needed pattern recognition and momentum resolution. The CMS detector uses a large number of discrete high-precision measurements to take advantage of its large magnetic field. Both detectors have very powerful vertexing capability for reconstructing the decays of long-lived particles.

The ATLAS detector uses a liquid argon electromagnetic calorimeter in a novel geometry to provide a finely segmented design. The liquid argon technique provides radiation hardness, good resolution, and very uniform response. The CMS collaboration is considering two calorimeter technologies, one based on scintillating plastic tiles, the second consisting of high-density crystals. The latter option would provide superb energy resolution, albeit at greater cost, taking advantage of the absence of any coil material inside the calorimetry. Both detectors contain hadronic calorimetry surrounding the electromagnetic calorimeters to provide hermetic coverage for jet and missing energy reconstruction.

The large toroidal magnet of the ATLAS detector provides a large field volume for accurate muon reconstruction without the need for many thousands of tons of steel. The CMS detector instruments the four layers of steel used to return the magnetic flux of the large solenoid in order to provide a muon spectrometer. Both detectors offer powerful muon measurement capability, even in the absence of inner tracking information, allowing reliable measurements at very high luminosities. 
These two general-purpose detectors use a diverse array of methods and technologies to provide the range of capabilities needed to explore the physics at the LHC. The U.S. community, with its extensive experience with SSC detector design and preparation, has much to offer to the LHC collaborations.

\section{PHYSICS AT THE LHC}

The physics questions and goals which can be addressed by the LHC are as compelling today as they were a decade ago when the SSC was first proposed. They include the elucidation of electroweak symmetry breaking, the search for supersymmetric and other new particles and the measurement of top quark properties.

1. Electroweak Symmetry Breaking. Based on what we now know, the study of electroweak symmetry breaking will be the most important physics objective of the LHC. The LHC, with its lower energy, does not quite guarantee the discovery of the mechanism of symmetry breaking, as the SSC did. Nevertheless, the LHC is certain to make substantial contributions to our understanding of this subject.

Extensive studies of how to detect the Higgs scalar in the minimal standard model have been carried out in recent years. The conclusion is that the LHC at $10^{34} \mathrm{~cm}^{-2} \mathrm{sec}^{-1}$ is capable of discovering the minimal Higgs for masses in the range of 80 to $800 \mathrm{GeV}$, its theoretical upper limit. In contrast, for a luminosity of $10^{33} \mathrm{~cm}^{-2} \mathrm{sec}^{-1}$ the region explored is somewhat reduced, so more aggressive search strategies must be used and safety factors are lost. At this luminosity, the mass region of $\mathbf{1 8 0}$ $\mathrm{GeV}$ up to about $600 \mathrm{GeV}$ can be covered in a reliable manner. The region between $130 \mathrm{GeV}$ and $180 \mathrm{GeV}$ appears to be covered if one assumes a good vertexing capability in the detector. The region below $130 \mathrm{GeV}$ seems to require a very high performance crystal calorimeter to isolate a plausible signal.

For the supersymmetric Standard Model, the implications of lower luminosity are more severe. At $10^{34} \mathrm{~cm}^{-1} \mathrm{sec}^{-1}$, at least one of the predicted Higgs bosons should be observable for most parameter values. At a luminosity of $10^{33} \mathrm{~cm}^{-2} \mathrm{sec}^{-1}$, it is possible that no supersymmetric Higgs bosons would be observed, which illustrates how valuable it is to utilize the full LHC design luminosity.

If there is no light Higgs particle, electroweak symmetry is probably broken by a new set of strong interactions. At a luminosity of $10^{33} \mathrm{~cm}^{-}$ ${ }^{2} \mathrm{sec}^{-1}$, such scenarios are very difficult to study at the LHC. For these 
types of models, $10^{34} \mathrm{~cm}^{-2} \mathrm{sec}^{-2}$ is essential. At the higher luminosity, resonant enhancements are probably observable, but nonresonant excesses might require many years of data taking and still not be convincing.

2. Supersymmetry. Supersymmetric theories predict new partners for all of the particles we know today. For supersymmetry to be relevant for the issue of electroweak symmetry breaking, the masses of these new particles must be less than about $1 \mathrm{TeV}$. The supersymmetric partners of the quarks and gluons are produced in hadron colliders with large cross sections. At the LHC, operating at $10^{33} \mathrm{~cm}^{-2} \mathrm{sec}^{-1}$, they should be observed up to masses of roughly $1 \mathrm{TeV}$ through their decays to missing energy plus jets or like-sign dileptons. These searches appear to remain viable at the full $10^{34} \mathrm{~cm}^{-2} \mathrm{sec}^{-1}$ luminosity, which leads to an ultimate mass reach of about $1.5 \mathrm{TeV}$, a factor of four or five above the reach of the Tevatron. The superpartners of the $W$ and $Z$ might also be observable via their decays to multiple-lepton final states over a more limited mass range. Thus, it is very likely that the LHC will confirm or exclude the existence of supersymmetry.

3. Top Quark Physics. Top quarks with a mass of around $170 \mathrm{GeV}$ will be pair-produced with a large cross section at the LHC. Indeed, the LHC is a veritable top factory, with more than $10^{7}$ top anti-top pairs produced in one year of $10^{33} \mathrm{~cm}^{-2} \mathrm{sec}^{-1}$ operation. The isolation of large, clean samples of top quarks is best accomplished by tagging the presence of long-lived $b$ quarks among their decay products. This becomes difficult when the luminosity of the machine is high, above $10^{33} \mathrm{~cm}^{-2} \mathrm{sec}^{-1}$. With such large samples, the precision of the top quark mass measurement is limited by systematic effects to about 3-4 GeV.

This large sample of top quarks allows accurate measurement of the top decay properties and powerful searches for rare or unexpected decay modes. For example, it is possible to discover various supersymmetric partners among the top decay products. Significant studies of top couplings can also be made. For example, measurements of the $W$ polarization provide an important test of the coupling of the top to the $W$.

4. Gauge Boson Physics. One of the deepest predictions of the electroweak theory is that the $W$ and $Z$ bosons have self-interactions beyond those expected in electromagnetism. Direct measurements of these interactions provide basic tests of the Standard Model. With the large samples of 
gauge boson pairs produced at the $\mathrm{LHC}$, even at $10^{33} \mathrm{~cm}^{-2} \mathrm{sec}^{-1}$, it will be possible to measure these couplings with a precision of several percent. This accuracy is better than that which will be obtained at LEP II.

Many unified theories predict the existence of additional gauge bosons. The LHC will search for heavy gauge bosons up to about 3-4 $\mathrm{TeV}$ at $10^{33} \mathrm{~cm}^{-2} \mathrm{sec}^{-1}$, and $4-5 \mathrm{TeV}$ at $10^{34} \mathrm{~cm}^{-2} \mathrm{sec}^{-1}$. It can also explore their couplings to quarks and leptons via asymmetries in the decay distributions, leading to important constraints on the structure of the underlying theory, if these new bosons were to be found.

5. Bottom Quark Physics. The LHC will produce roughly $10^{13} b$ quark pairs per year at a luminosity of $10^{33} \mathrm{~cm}^{-2} \mathrm{sec}^{-1}$. This represents a factor of $10^{5}$ more $b$ quarks than are produced at an electron-positron $b$ factory and a factor of $10^{2}$ more than the Tevatron will yield in the Main Injector era. However, the hadron collider environment presents severe challenges in harvesting the benefits of such large samples.

Nevertheless, the general purpose detectors with their high-performance tracking systems will be capable of further exploring charge parity (CP) violation and rare decays. A dedicated collider $b$ experiment would run at lower luminosity, but with particle identification and high-resolution vertexing it should provide a superb laboratory for very precise $C P$ violation measurements and exploration of the properties of the strange $B$ meson.

The physics program for the LHC operating at $10^{33} \mathrm{~cm}^{-2} \mathrm{sec}^{-1}$ is very rich, and represents a significant advance along the energy frontier. At the design luminosity of $10^{34} \mathrm{~cm}^{-2} \mathrm{sec}^{-1}$, many additional physics studies will be possible, particularly in the area of electroweak symmetry breaking.

\section{E. THE HADRON ENERGY FRONTIER BEYOND THE LHC}

The history of elementary particle physics has been one of surprises. As accelerator energies have increased, each new energy scale has yielded unexpected insights into the underlying structure of the physical world. While the discoveries yet to be made at today's frontiers, and those reached by the LHC a decade hence, will make our expectations for physics at the next-higher energy scale more concrete, past experience suggests that we need to begin planning now for that far horizon. In this respect, it is worth noting that the planning that ultimately resulted in the SSC and LHC proposals began in 1978 and 1979 with two workshop meetings of the International Committee on Future Accelerators (ICFA). These studies focused on the $10 \mathrm{TeV}$ scale 
for protons. A first look at possibilities at the $100 \mathrm{TeV}$ scale is embodied in the reports from a series of workshops on accelerators and detectors held at the Ettore Majorana Centre in Erice starting in 1988.

The technical challenges to reaching the $100 \mathrm{TeV}$ scale are formidable. In this new regime, the behavior of any proton collider, based on the synchrotron principle, will for the first time, be dominated by synchrotron radiation. The large power carried away from the interaction point in reaction products will tax detector and accelerator components in the extreme. Innovative accelerator science and technology will be required to achieve and maintain the needed beam brightness and intensities. Before research and development on technical components can proceed, a thorough economic and technical optimization study must be carried out.

An undertaking of this scale requires a regional or global collaboration from the beginning. The likely duration of such preparatory work argues again for the need to begin soon. Current global collaboration on electron-positron collider research and development could well serve as a model for starting work on an even more powerful hadron collider.

Physics at Hadron Colliders Beyond the LHC. The LHC represents a major step on the hadron frontier, but it is only a step. It is clearly too soon to say at what energy the next hadron facility should be built. Results from the LHC may suggest that a machine much larger than the SSC is warranted. For example, if the LHC were to uncover hints of a stronglyinteracting symmetry-breaking sector, more energy will surely be needed to reveal its detailed principles.

For the sake of discussion, let us consider an energy of $100 \mathrm{TeV}$ for such a future collider. This gives roughly the same extension in reach relative to the LHC as the LHC gives relative to the present Fermilab Tevatron. Such a collider could allow a full exploration of a stronglyinteracting symmetry-breaking sector, as well as a considerable extension of our ability to probe for a deeper layer of structure below that of the quarks and leptons if these objects were to be composite.

At ultrahigh energies, the scattering of longitudinal W's provides an important probe of electroweak symmetry breaking. For this process, if the scattering is strong, the signal increases by roughly a factor of 20-50 in the $1-2 \mathrm{TeV}$ mass region when going from a $14 \mathrm{TeV}$ to $100 \mathrm{TeV}$ (assuming constant luminosity). The discovery potential is probably even greater because the backgrounds do not increase as rapidly as the 
signal. Therefore a $100 \mathrm{TeV}$ machine would allow detailed studies of the new strong forces, similar to the studies of meson and baryon spectroscopy carried out in the 1950s and 1960s.

A $100-\mathrm{TeV}$ hadron collider would greatly extend our reach in other areas too. Studies suggest that it would be sensitive to the indirect effects of new physics up to scales of $50 \mathrm{TeV}$ for a luminosity of $10^{33} \mathrm{~cm}^{-2} \mathrm{sec}^{-1}$, and perhaps $70 \mathrm{TeV}$ for ten times this luminosity. The discovery range for new particles would be extended to perhaps $20 \mathrm{TeV}$ or more.

\section{F. THE ENERGY FRONTIER AT ELECTRON-POSITRON COLLIDERS}

A next-generation electron-positron collider can address many of the important physics goals and questions brought forward in Chapter III. A great variety of final states are produced in electron-positron annihilation at rates that can be accurately predicted and normalized. The simplicity of the annihilation process allows particles and interactions to be isolated for study, and new and unexpected phenomena to be identified. The recent development of highly-polarized electron beams enables electron-positron colliders to be used for unique studies that can help disentangle different particle interactions. These features combine to make electron-positron annihilation an important tool for exploration of the $\mathrm{TeV}$ mass scale.

1. Higgs Physics. Experiments at an electron-positron collider can make definitive searches for Higgs scalars and ensure that we understand the spectrum and interactions of any such particles that exist up to the energy reach of the machine. Production of boson pairs is substantial and a good process for the discovery and study of Higgs scalars with masses up to $400 \mathrm{GeV}$ or so. At higher masses, the process $\mathrm{e}^{+} \mathrm{e}^{-} \rightarrow v \overline{\mathrm{v}} \mathrm{H}$ becomes more useful. Various decays of Higgs particles can be isolated from backgrounds, and Higgs couplings determined from measured production and decay rates would discriminate between possible models of electroweak symmetry breaking.

2. Supersymmetry. Supersymmetry partners can be produced at an electron-positron collider up to the energy limit of the machine, and their masses and quantum assignments determined. Even particles that might decay to only neutral weakly interacting final states would appear in missing-mass spectra. Polarization of the electron beam can allow basic properties of supersymmetry to be tested and revealed, providing far-reaching tests of the theory. 
3. Top Quark Physics. The properties of top quarks can be investigated in electron-positron annihilation both near threshold for production of top pairs, and at higher energies, where individual quarks can be well isolated. Precision determinations of the strong coupling constant and of the top mass (with an error of $0.5 \mathrm{GeV}$ ), and an important measurement of the top decay width, can be made at threshold. Above threshold, control of the incident electron polarization will allow detailed study of the structure of the couplings of the top quark to the $Z$ and $W$ bosons. Possible top decays into Higgs or supersymmetric particles can also be clearly isolated from backgrounds.

4. Gauge Boson Physics. Large samples of $W^{+} W^{-}$pairs, and the use of polarization, would enhance the study of longitudinal boson states that are the remnants of electroweak symmetry breaking. The structure of the $W$ can be measured with accuracies of a few percent at $500 \mathrm{GeV}$, and the rapid growth with energy of the production of longitudinal $W^{\prime} s$ yields a factor of ten improvement as the energy is increased to $1 \mathrm{TeV}$ and beyond. Additionally, an electron-positron collider can probe the self-interactions of gauge bosons, and it may be possible to measure the Higgs scalar self-coupling. This set of measurements would provide important insights into the gauge structure of the Standard Model.

New gauge interactions and corresponding bosons may exist, and measurements made at $1 \mathrm{TeV}$ will be sensitive to neutral bosons with masses as large as $4-5 \mathrm{TeV}$. Complete studies of the pattern of asymmetries produced in a variety of leptonic and heavy quark final states will provide unique and significant constraints on the underlying gauge symmetries of any new interaction.

\section{Physics Opportunities at High Energies. An electron-positron collider} capable of reaching energies of $1.0-1.5 \mathrm{TeV}$ has the potential to probe further the origin of mass. The breaking of electroweak symmetry is embodied in the presence and interactions of the longitudinal states of the massive $\mathrm{W}$ and $\mathrm{Z}$ gauge bosons. Re-scattering of final-state bosons in the reaction $\mathrm{e}^{+} \mathrm{e}^{-} \rightarrow \mathrm{W}^{+} \mathrm{W}^{-}$is a source of such interactions, and precision analyses can be sensitive to resonant structure at masses up to 5-6 TeV. The use of polarized electrons can allow nonresonant scattering of longitudinal bosons to be established above the background. The reaction $e^{+} e^{-} \rightarrow v \bar{v} W^{+} W^{-}$is another source of boson scattering, and Higgs resonances with masses up to approximately $800 \mathrm{GeV}$ can be studied. With the highest energies and luminosities, the presence of nonresonant $\mathrm{W}$ scattering might be established. 


\section{G. LINEAR COLLIDER DEVELOPMENT}

The basic components of any linear collider are those already incorporated in the Stanford Linear Collider (SLC) at SLAC. Trains of bunches of electrons and positrons are created, condensed in damping rings, accelerated to high energy, focused to small spots, and finally collided. Constrained by the need to control the number of photons emitted during the beam-beam interaction, the luminosity of the collisions is determined simply by the power carried by the beam and the beam size at the collision point. High luminosity is achieved by high beam powers or small beam spots. These two parameters pose different, and in many cases conflicting, challenges to the accelerator physicist. Today, no feasible means for obtaining the required energy and luminosity is in hand. However, several technology choices made around this set of challenges are being pursued by collaborations of research groups around the world. The use of conventional roomtemperature accelerators powered by high-power sources at S-Band ( 2.8 $\mathrm{GHz})$ or X-Band (11.4 GHz) frequencies; a two-beam accelerator (CLIC) at still higher frequency; and the use of superconducting linacs (TESLA). It is hoped that in a few years one of these approaches will emerge as a viable technology for a practical linear collider.

Continued use of well-known S-Band components offers a conservative path to accelerators with beam energies of several hundred $\mathrm{GeV}$. The SLC is a prototype for such a collider. With accelerating gradients limited to $20-30$ million volts per meter $(\mathrm{MV} / \mathrm{m})$, however, upgrade of the machine to $\mathrm{TeV}$ energies is more difficult to achieve, and such an accelerator would be more expensive than machines capable of higher gradients. Research at the Deutsches Elektronen Synchrotron (DESY) in Germany, KEK in Japan, and SLAC centers on optimization of this technology and understanding its limiting factors.

A more natural match to the $\mathrm{TeV}$ energy region is made with a choice of X-Band components. This requires development of new sources of microwave power, but will provide gradients of 50-100 $\mathrm{MV} / \mathrm{m}$. To create and collide nanometer beam spots is the greatest challenge to this technology. The technical risk will be greater than that incurred at S-Band, but the capital costs of initial construction at 500 $\mathrm{GeV}$, and the cost to upgrade the machine to $\mathrm{TeV}$ energies, are expected to be lower. Much work remains to be done on these technologies, but prototype components are now in hand. Fully engineered GeV-scale accelerators under construction at KEK and SLAC will allow optimization of accelerator systems, and provide experience with beam operations. A next-generation damping ring, jointly designed by KEK 
and SLAC, is under construction at KEK to explore preparation of trains of bunches suitable for an X-Band machine. These facilities are expected to be completed during the next $2-3$ years.

Superconducting microwave structures have been used for some time to accelerate beams of particles. The Continuous Electron Beam Accelerator Facility (CEBAF) linac, and the development of cavities for TRISTAN and LEP II, represent the state-of-the-art in large-scale applications of superconducting accelerating systems. The ability of superconducting structures to store electromagnetic energy for long periods of time allows designs with high beam powers and correspondingly larger beam sizes. However, the $25 \mathrm{MV} / \mathrm{m}$ accelerating gradient needed to keep the linac to reasonable lengths is large compared to the 5-7 MV/m achieved in existing accelerators; and present costs of superconducting systems are excessive for large-scale use. Fermilab and Cornell are part of an experienced international collaboration that has begun to attack these problems. Advances in fabrication techniques and the use of pulses of high power to condition cavities have been pursued at Cornell, where gradients of $25-30 \mathrm{MV} / \mathrm{m}$ have been achieved. Work continues to reduce costs of the required cryogenic systems, and construction of a test facility now begun at DESY is expected to be completed in 1997.

The spot size at the collision point of a future collider is required to be 10 to 100 times smaller than achieved at the SLC. The international Final Focus Test Beam Collaboration has designed and built a prototype beamline at SLAC to address this problem, and a vigorous experimental program is under way in 1994.

A foundation for an interregional effort to construct and use a nextgeneration linear collider exists in the form of collaborations at work on the accelerator physics and technology. As the pace of this work has quickened, the institutions involved have established an Interlaboratory Collaboration on Research and Development Towards TeV Linear Colliders to provide a forum in which technical options can be evaluated and presented for consideration by the international community. This represents a significant advance towards realization of a collider as an international project. 
Given the large size, complexity, and cost of accelerators, technological advances will be required in order to achieve further significant advances in energy for future research. Such innovations require long lead times.

Several concomitants of increasing energy need attention. Already in currently planned colliders, the power carried away from the interaction point by the reaction products of dissipative processes ranges from kilowatts to tens of kilowatts, which poses a severe challenge to detector and accelerator components alike. In higher-energy accelerators, these powers will grow. As yet, no scheme has been put forward to cope with this power rise.

Currently planned machines will consume roughly one hundred megawatts of power. Without technology improvements, power requirements will scale up faster than energy. Thus, substantial improvements in electric-power-to-beam-power efficiencies will be necessary. Total beam currents will likely need to rise, putting further demands on stability control in the face of higher impedances presented by higher-energy accelerators.

Cost was a pivotal issue for the SSC and is likely to be so for future accelerators and detectors. Without technological innovations, these costs will rise more rapidly than the energy of the machine. There is an obvious need to reduce cost per unit beam energy, either through evolutionary improvements to existing technologies or by entirely new schemes.

An evolutionary approach to improvement of synchrotron and microwave linear accelerators has served us well in the past. This approach has led to the hadron and electron-positron colliders now in use and under construction. Through use of newly developed materials, advances in automated manufacturing techniques and machinery, as well as in electronics, cryogenics and control technologies, the costs per unit beam energy and current have continually decreased. The new accelerators have been heavily dependent on continuing advances in the understanding of basic accelerator and beam physics, which have enabled exploitation of these technological developments. 
In the past dozen years, extensive effort has been invested in development of new acceleration systems and systems for accelerating particles other than protons and electrons, such as muons and gammas. Between 1982 and early 1994, more than ten workshops on advanced accelerator concepts have been held. Most were international in scope. Many ingenious ideas have been put forward, and some very encouraging experimental results have been reported. As yet, none of these new approaches has demonstrated all of the characteristics needed for a high-energy physics accelerator: stageability to very high energy, high electric-power-to-beam-power efficiency, high beam brightness and narrow energy spread, beam position stability and cost advantage with respect to present standards. With time and support, these deficiencies may be eliminated.

Which approaches will be most effective in the long run remains to be seen. The needs and challenges are clear. The community must reemphasize its efforts to provide practical vehicles for accelerator-based particle physics beyond the present frontiers. 


\section{PARTICLE PHYSICS WITHOUT ACCELERATORS}

Throughout the history of particle physics, important contributions to our understanding have come from experiments that do not rely on accelerators as the source of particles. In fact, the high-energy extraterrestrial particles known as cosmic rays, which revealed new forms of matter to researchers in the first part of this century, provided inspiration for building particle accelerators. Since then, a number of methods drawing on concepts and techniques from other areas of the physical sciences have been used to explore some of the central questions of our field. For example, the impressive precision of contemporary atomic physics has allowed us to measure parity nonconserving effects and put stringent limits on charge parity $(\mathrm{CP})$ violation. Nuclear reactors and very low radioactive background experiments deep underground have been used to study neutrino masses, and large underground detectors have investigated the lifetime of the proton. Accelerator and non-accelerator particle physics frequently share tools and experimental techniques-and sometimes even the same inquiring minds.

While accelerators can deliver larger numbers of particles with a specific energy, non-accelerator experiments can selectively probe territory beyond the reach of accelerators that we know how to build today. Supernovae shocks and black holes produce particles of higher energy than we can make. Dark matter experiments are probing wider mass ranges than existing accelerators. Proton decay experiments give us information about physics on an energy scale too high for us to reach now by any other means.

\section{A. The Relation Between Particle Physics AND Astrol'hysics}

In this chapter, we focus on particle astrophysics, a relatively new subfield of particle physics that shares many intellectual frontiers with high-energy physics. Although the experiments in particle astrophysics are not directly associated with accelerators, the two disciplines have common roots and many physicists take part in both. There are some 250 experimental particle astrophysicists.

The questions addressed by particle astrophysics are among the most fundamental puzzles of science today. Why do we live in a universe that is made of matter and not a mix of matter and antimatter? What is the nature of the dark matter that constitutes more than $90 \%$ of the mass of the universe? If indeed dark matter can be proven to be nonbaryonic, this would be the ultimate Copernican revolution: not only are 
we not at the center of the universe, but we are not even made of what it is mostly made. What is the origin of the large scale structure of the universe? Are quantum phenomena the seeds for tiny density enhancements that have now become galaxies? How do stars shine and die, and what do the neutrinos we observe tell us about these mechanisms? What are the giant accelerators in the cosmos and what can we learn about fundamental physics by studying them?

It should be noted that in many of these problems, it is difficult to distinguish between what is particle physics and what is astrophysics; to take the example of solar neutrinos, is the observed deficit due to some process in the sun that we do not understand or is it due to a small mass of the neutrino? The culprit now appears to be the neutrino. If this is confirmed, it would be of great benefit both for astrophysics, with the confirmation of one of its most basic foundations, the energy production in stars, and for particle physics, as an indication of physics beyond the Standard Model.

\section{B. EXAMPLES OF FUtURE PROSPECTS AND OPPORTUNITIES IN PARTICle ASTROPHYSICS}

Particle astrophysics has three different but overlapping components: particle cosmology, neutrino astrophysics, and ultra-high energy astrophysics.

Particle cosmology is aimed at understanding the early universe. Particularly interesting here is the quest to identify the mysterious dark matter that appears to constitute more than $90 \%$ of the matter in the universe. A new generation of very sensitive low background experiments using novel cryogenic techniques for active background rejection is coming on line and promises to begin to test the hypothesis that the lightest supersymmetric particles constitute dark matter. Similarly, searches are in progress for the axion, another dark matter candidate. Astrophysics measurements such as the measurement of the anisotropy of the microwave background, the mapping of the large scale structure in the universe, or the measurement of space geometry have significant impact in terms of deciphering potential phase transitions in the universe induced by particle physics, and the role of quantum fluctuations in the formation of structure.

Neutrino astronomy started in the 1960 s when first measurements of the flux of neutrinos emitted by the sun turned out to be well below expectations. The combination of Kamiokande, a proton decay experiment that was upgraded to measure solar neutrinos, and two new radiochemical experiments (SAGE in Russia and GALLEX in the Gran 
Sasso Laboratory in Italy) that are sensitive to the main nuclear reaction in the sun, now generally confirms our understanding of energy production mechanisms in the sun and points to the intriguing possibility of neutrinos having a very small, but nonzero, mass. These conclusions should be further tested by two new experiments which will become operational in 1996, Sudbury Neutrino Observatory in Canada, and SuperKamiokande (which doubles as a proton decay detector) in Japan.

In addition to these two international collaborations, American physicists are involved in the development of a sophisticated experiment, Borexino, which will also be installed in Gran Sasso and will probe intermediate-energy solar neutrinos. This field of neutrino astronomy came of age in 1987 with the detection of the neutrinos from a supernova in three detectors across the world. This observation spectacularly confirmed our theoretical understanding of stellar collapse and the role played by the neutrinos in the process. Should a supernova occur in our galaxy during the operation of the solar neutrino detectors soon coming on line, important information will be obtained on the mass of the muon and tau neutrinos.

Ultra-high-energy astrophysics tries to understand the many violent phenomena occurring in the cosmos and to determine the mechanisms responsible for the acceleration of cosmic rays. Among the problems poorly understood are the relative roles of electromagnetic and hadronic acceleration processes around such compact objects as neutron stars, stellar black holes, or the massive black holes hypothesized to be at the core of galactic nuclei; the composition of cosmic rays; the source of gamma ray bursts and the origin of the very-high-energy cosmic rays. Potentially critical to the understanding of the first puzzle is the current development of techniques using polar ice (AMANDA) or the deep ocean water (DUMAND) as a medium for the detection of the veryhigh-energy neutrinos which would be produced by hadronic processes. Techniques that originated in particle physics are used in astrophysical explorations: devices such as atmospheric Cerenkov telescopes and water Cerenkov pools can usefully complement gamma-ray satellite observations. Experiments study energetic muons created by highenergy cosmic rays at large underground multi-purpose installations such as Soudan in Minnesota and Macro in Italy, mainly designed to search for proton decay and monopoles, respectively. Finally, recent detection by the Fly's Eye detector in Utah of an event with energy in excess of $10^{11} \mathrm{GeV}$ gives a new incentive to develop detectors that can greatly improve the sensitivity to these fascinating high-energy events, some of which may originate outside our galaxy. 
The financial support to non-accelerator particle physics (including particle and nuclear astrophysics, proton decay, neutrino experiments without accelerators and atomic measurements motivated by particle physics), currently totals about $\$ 40 \mathrm{M}$ annually; roughly two-third comes from the Department of Energy, and most of the remaining support comes from the National Science Foundation. About one-half of these funds are provided by the high-energy physics program in both agencies.

Particle astrophysics addresses issues that bear on the whole field of particle physics. It is reasonable to anticipate that the number of particle physicists working in this field will grow during the coming decade and that the devices for further advancing this basic field will also grow in size and cost, estimated to be in the range of several tens of millions of dollars for some of the facilities discussed above.

Strong collaboration among relevant U.S. funding agencies, such as the Department of Energy, the National Science Foundation and the National Aeronautics and Space Administration, will be important for the future of this field. The agencies should review the scope and means of developing a realistic long-term vision for the U.S. program and of evaluating collaboratively the appropriate cross-agency projects. 


\section{OUR VISION OF THE FUTURE}

Three essential elements must be present for the U.S. program in particle physics to remain a strong and competitive leader in the field. First, we must do the best possible physics in the near term, using the excellent accelerators and detectors that exist and are currently being upgraded in the United States, and also abroad when important opportunities exist. Second, we must create opportunities to open new research frontiers, through a vigorous program of advanced accelerator and detector research and development. Advances in technology realized by such a program, together with advances in scientific understanding that are the fruits of current experiments, will shape priorities for doing the best possible physics in the long term. Third, we must collaborate in research that will explore promising new territory on the energy frontier, using accelerators and detectors either within the United States or abroad. At this time, that implies joining with the European Laboratory for Particle Physics (CERN) at the Large Hadron Collider (LHC).

\section{A. USING CURRENT RESOURCES}

Existing accelerators and detectors, together with their upgrades that are now under way provide the United States with marvelous instruments that can ensure our role as a world leader in high-energy physics for the next ten to fifteen years. However, there must be a sufficiently strong and stable operating budget to permit a diverse and flexible research program with timely opportunities to do important physics. Such a program, in turn, would provide opportunities for younger scientists to grow and develop, and it would ensure a desired influx of the best of them into the field. If the construction of current upgrades proceeds on schedule, and their healthy use is supported, before the end of this decade we will be able to probe more deeply into such important issues as the properties of the top quark and the origin of charge parity $(\mathrm{CP})$ violation. We will also be able to respond, with flexibility, to new experimental opportunities.

It is likely, as the program unfolds, that future results will point to new opportunities at our existing domestic laboratories. The future might well include the evolution of the experimental programs beyond the accelerator upgrades now in progress. However, further upgrades should be pursued only if they can be justified within the context of the world program of high-energy physics measurements, or if they enhance the ability of the domestic program to advance future international projects. 
For many decades, research and development on hadron and electron-positron accelerators and their detectors has paced progress in advancing particle physics. Continued progress in our ability to address issues in particle physics depends on further advances in the instruments we build. Today, there exist important advanced research and development programs aimed at cost-effective accelerator technologies for the machines of the next century. For example, an international collaboration is at work on accelerator physics and technology development for a next-generation linear electron-positron collider. For hadron colliders, going beyond the energy of the Superconducting Super Collider (SSC) in a practical manner will require further advances in accelerator technology. As discussed in Chapter V, more speculative ideas are also being pursued for new concepts in accelerators.

Although some of these research and development efforts promise to lead to financially and technically feasible designs, it is not now known which options will provide the next step in understanding. A healthy long-term future for particle physics in the U.S. must be built on a solid foundation of accelerator and detector research and development in this country.

C. TAKING THE NeXT Step: COllaboration ON THE LHC

The LHC at CERN currently offers the most promising prospect to advance the high-energy frontier beyond the Tevatron. Although the LHC will not reach as far into unexplored regions as the SSC would have, it is certain to permit a major step in our understanding of nature. (See the discussion in Chapter V.)

Completing the research and development for this accelerator and its detectors, as well as building and using these instruments, will be a major technical challenge, a challenge that is important for U.S. particle physics. As a scientific partner in this venture, the U.S. would be able to use the expertise gained from preparations for the SSC to make a major step along the path to higher energies. Becoming a partner in the construction of this accelerator would also provide valuable experience in international collaboration on high-energy physics at a new level, an important step toward developing a framework for participating in large, international scientific collaborations. 
Supporting a productive, flexible, and diverse near-term program that includes investment in research and development for the instruments of the long-term future, as well as collaboration at the frontier available at the LHC, will build a strong case for this country hosting other large scientific collaborations, including an internationally created accelerator. The health of our program and our infrastructure, our academic and technical strength, and our reliability as a collaborator on international projects will all be weighed in a future decision of where to build such a project.

In planning for the future, we emphasize that a healthy program is not and should not be restricted to experiments involving accelerators. Scientifically compelling issues concerning the properties of elementary particles can, and in some instances can only, be addressed through other techniques. Experiments that do not rely on accelerators can intellectually complement studies performed at accelerator laboratories and add important flexibility, diversity, and training opportunities to the U.S. program.

The U.S. high-energy physics program has long been a source of dramatic scientific progress and national pride, and a symbol of international collaboration. Building on the superb quality of its institutions of higher learning and research, and the generous support of the American public, the U.S. program has been a world leader at the frontiers of discovery. If our recommendations are implemented we. believe it will remain so well into the twenty-first century. 
(BLANK) 


\section{REALIZING THE VISION}

In the preceding chapter, we described the three essential ingredients for the U.S. particle physics program to fulfill our vision that it remain vital and among the leaders, worldwide, beyond the next decade: a flexible and diverse research effort with strong use and support of domestic accelerators and detectors; vigorous research and development in advanced accelerator and detector technologies; and continued participation at accelerators on the highest energy frontier, for which the current best opportunity beyond the Tevatron is through collaboration in the Large Hadron Collider (LHC) project at the European Laboratory for Particle Physics (CERN). In this section we discuss strategies and budgetary implications for achieving this vision. First, however, we will review the current problems and issues faced by the U.S. high-energy physics program, to better illuminate what is required to achieve our goal of a strong, productive, and internationally competitive particle physics program for the U.S., extending two decades and beyond into the future.

\section{A. PRoblems AND ISSUES IN THE CURRENT U.S. PROGRAM}

The current U.S. program, described in chapter IV, relies on a powerful set of accelerators and detectors to investigate many of the critical questions in particle physics. With funding that is adequate to support our investment in these resources and the improvements that are now being carried out, the U.S. high-energy physics program would remain a world leader for a decade or more. However, as we outline below, the U.S. high-energy program is severely strained and badly out of balance as it enters FY1995. This is the consequence of a continuing trend of reductions in its budget at the same time as the program is committed to planned improvements that were undertaken to ensure the program's strength and productivity through the coming decade. As a result, the U.S. particle physics community faces severe challenges, both in retaining a prominent role in this fundamental area of science and in attracting and retaining some of the best young scientific minds in the country. To further elaborate:

1. With the cancellation of the SSC, we have lost the project on which a decade of our planning for the future has been based. Consequently, many excellent scientists have suddenly found their plans for research dashed, and their considerable achievements in accelerator and detector technology, at least temporarily, in limbo. Research and leadership opportunities for young people have been sharply 
reduced. As a result, some of the best researchers and students have become profoundly discouraged about the long-term prospects for a productive future in this field.

2. The cumulative effect of painful budget reductions that the community accepted in anticipation of the SSC severely strains the ability of the U.S. high-energy physics program, without the SSC, to realize its great scientific potential. There has been an overall decline in the budget for the national program, aside from the SSC. In Figure 1, we show the history of the annual budget for the highenergy physics program and for the SSC, in FY1995 dollars. ${ }^{1}$ Of particular importance is the effect on the research program, which is primarily supported by the Operating and Equipment budgets shown as the black and the lower hashed bands. This research support, \$547M in FY1990, has shrunk to \$510M in the President's budget submission to Congress for FY1995. When inflation is included, this represents a loss in FY1995 of about $\$ 135 \mathrm{M}$, or $20 \%$ of the buying power of the annual research budget. The portion of this budget appropriated to support the university-based research groups also dropped in the same proportion-i.e., 20\%-during this time.

In addition, the national program lost funds over the past three years (\$58M in FY1993, \$14M in FY1994, none expected in FY1995), that came from the SSC project and from the Texas National Research Laboratory Commission (TNRLC). These funds were used to support SSC-related research efforts at universities and national laboratories across the country.

Decreases in funds at the national laboratories have led to reductions in the operation of accelerators, delays and curtailment of research programs, and significant reductions in staff. These reductions have already meant significant losses to the U.S. program in terms of numbers of experiments that can be performed and of delays in completing those in progress due to loss of running time and staff support.

${ }^{1}$ This figure does not include the National Science Foundation (NSF) funding for the Cornell Electron Storage Ring and other NSF-supported university research groups. This NSF support adds approximately $\$ 50 \mathrm{M}$, or $7 \%$, to the total spending in high-energy physics in FY1995. These NSF funds have been flat in then-year dollars, corresponding to roughly a $15 \%$ loss in buying power due to inflation since 1990. 
3. Planned upgrades of existing accelerators that provide important new research opportunities place new demands, even as budgets have shrunk. The construction projects to upgrade existing facilities and open important new research opportunities toward the end of this decade- the Fermi National Accelerator Laboratory (Fermilab) Main Injector, the Stanford Linear Accelerator Center (SLAC) $B$-factory, and the upgrade of Cornell Electron Storage Ring (CESR) at Cornell-have taken on added importance with the cancellation of the SSC. The scheduled Department of Energy construction costs of the Fermilab Main Injector and the SLAC $B$-factory are shown in Table B. If their funding schedule is maintained, the construction costs reach their peak in FY1996, drop significantly in FY1998, and are zero in FY1999.

4. An additional strain on the budget is placed by some fifteen new university-based experimental high-energy physics research groups funded by the Department of Energy's Division of High Energy Physics, largely in anticipation of research opportunities at the SSC. This growth of about $10 \%$ in the number of experimental groups in the Department of Energy's high-energy physics program extended research opportunities to geographic regions previously underrepresented in the particle physics program.

5. The future resides with the younger members of the field, yet it is precisely this group that has been hurt most by shrinking support. The cancellation of the SSC and declining budgets have led to a scarcity of new job opportunities. While there has been a significant buildup in the number of young physicists in temporary positions, again in anticipation of the SSC, prospects for permanent positions are bleak, and the careers of many young physicists who would be assuming leadership roles across the next decade and beyond are in serious jeopardy.

\section{B. STRATEGIES FOR REALIZING THE VISION}

Two important points emerge clearly when we look at the budgetary profiles for implementing our vision. The first is that the greatest immediate needs are for revitalizing the current program to serve as a healthy and balanced base on which to build a future. The second is that continuing an effective American presence on the high-energy frontier, which is essential for the long-term vitality of the U.S. program, does not make a large, immediate demand on the budget. What it does 
need is an early "go" decision by the U.S. government to enable the scientists to plan effectively for participating in the research and development toward construction of the accelerator and detectors at the LHC.

The build-up in sizable U.S. spending levels as part of the LHC collaboration can be phased in as the current commitments by the Department of Energy to complete the two main upgrades of our current facilities-the Main Injector at Fermilab and the $B$-factory at SLAC-wind down. The planned spending profiles for these two initiatives are given in Table B in terms of constant value FY1995 dollars. The construction costs drop rapidly after FY1997. In addition, current Department of Energy plans call for the Alternating Gradient Synchrotron (AGS) injector at Brookhaven National Laboratory (BNL) to be incorporated into its nuclear physics budget in FY1999, thereby relieving the high-energy physics program of an estimated $\$ 40 \mathrm{M}$ annual cost that is part of its current operating responsibility.

Table B

Funding to Complete Projects

Millions of FY1995 Constant-Value Dollars

FY94 FY95 FY96 FY97 FY98

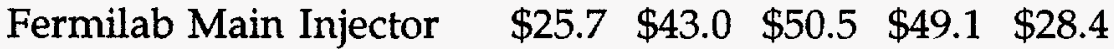

SLAC B-factory $\quad \$ 37.1 \quad \$ 44.0 \quad \$ 50.5 \quad \$ 42.5$

These reductions in budgetary obligations of the U.S. high-energy physics program were anticipated in the planning for physics research at the SSC, scheduled to start by the end of this decade. We illustrate with two funding scenarios that use of the funds resulting from these reductions will provide much needed flexibility to meet both short-term and long-term program needs and that, with modest budgetary commitments, the U.S. can support a program for realizing our vision.

1. Budget Scenario A: A temporary, partial restoration of funding levels.

The motivation for this funding profile is to restore an important measure of strength and responsiveness to the U.S. high-energy physics research program, starting in FY1996, without further delays in the scheduled completion dates of the upgrades at Fermilab (the 
Main Injector) and at SLAC (the $B$-factory). The need we identified is a temporary bump in the budget of approximately $\$ 50 \mathrm{M}$ per year for three years, FY1996 through FY1998, followed by a return in FY1999 to the FY1995 level (in constant-value FY1995 dollars), as shown in Figure 3.

One can present a variety of arguments in support of such a bump in the budget. This bump is a modest request, but it would be crucial in revitalizing the ongoing research program. This increase in FY1996, above the President's FY1995 budget request, would recover about $37 \%$ of the loss in annual support for operations and equipment over the past five years. It would restore the research budget in high-energy physics approximately to its FY1994 level in buying power, thereby permitting existing resources to be much more efficiently and productively used. At the same time, the upgrades currently being built at Fermilab and SLAC could proceed on schedule. The loss of the SSC and its anticipated rich opportunities for research further increases the importance of gaining timely access to the new research frontiers that these upgrades will open.

A major benefit of this three-year bump is that there would be no need for further sharp reductions in the scope of the current program. It would also allow further important increases in funds available for the research program in FY1997 and FY1998, as the construction cost for the two upgrades decrease, as shown in Figure 3 . These funds would be available for a growing U.S. involvement in the LHC program and for other initiatives and program needs that may emerge in the meantime. In FY1999, the proposed upgrades will be completed, if funded on schedule, and additional program funds will become available from the planned transfer of AGS operations at BNL to the nuclear physics budget for running the Relativistic Heavy Ion Collider (RHIC).

The simple message of Figure 3 is that the three-year bump in the total high-energy physics budget over the President's FY1995 budget request allows modest but very important support for the research program, which will help sustain it through the construction years of the two upgrades. The figure also shows that if this three-year increase is followed by a drop back to a total budget with constant buying power at the FY1995 level, there will still be important flexibility in the research budget to permit significant and growing participation in the $\mathrm{LHC}$, as well as to strengthen and diversify the national program. With this bump, funds would also be available to 
support a continuing program of vigorous research and development on accelerator technology in search of new avenues to pursue the critical physics issues discussed in chapters III, IV, and V.

Given this three-year supplement, the subpanel recommends that the U.S. government declare its intention to join in the collaboration constructing the LHC at CERN and initiate negotiations toward that goal. With such a commitment to the future, it will be important for the U.S. to sustain the research and development effort on magnets and detectors already begun for the SSC and to redirect it toward the L.HC. We foresee expenditures starting in FY1995, at the level of $\$(5$ to 10$) \mathrm{M}$, and $\$(10$ to 15$) \mathrm{M}$ in FY1996, with larger expenditures thereafter. Continuing build-up to a steady level of $\$ 60 \mathrm{M}$ per year, starting in FY1999, would result in a total of about $\$ 400 \mathrm{M}$ over nine years ${ }^{2}$ by the end of FY2003. We expect that a large fraction of this $\$ 400 \mathrm{M}$ would be spent in the United States on, for instance, building special magnets and equipment for the interaction regions and the large detectors. This would constitute a serious, effective, and important U.S. investment of great value to both the U.S. and the LHC program itself.

In summary, a flat budget at the level of effort proposed in the President's budget for FY1995, plus a temporary three-year supplement of $\$ 50 \mathrm{M}$ per year, will enable the U.S. to achieve the following goals in an important and effective, if limited, measure:

i. Repair some losses to the current program;

ii. Complete the Main Injector and B-factory upgrades as scheduled;

iii. Support a more healthy and vigorous U.S.-based research program through the decade ahead;

iv. Support research and development for future advances on the high-energy frontier;

v. Enter into meaningful and timely participation in construction of the LHC accelerator and detectors to provide research opportunities at the energy frontier for U.S. physicists.

\footnotetext{
${ }^{2}$ There is nothing magic about the figure of $\$ 400 \mathrm{M}$. It is introduced only to indicate the scale of possible total involvement under this budget assumption.
} 
vi. Take the first essential step toward international collaboration in building future accelerators;

vii. Make important use of the scientific and technical progress achieved in preparation for the SSC.

The LHC currently offers the best practical means for effective U.S. participation in accelerator and detector research and development and in physics on the high-energy frontier beyond the Tevatron. It is an important opportunity for the U.S. program and should be endorsed with timely support. The scale of involvement in the LHC contemplated above is fully compatible with the vision presented in chapter VII for the long-term future of the U.S. program.

However, despite the impressive list of goals that could be achieved with the proposed three-year budget supplement, this would nevertheless be a significantly reduced program when compared with the high-energy research program supported five or ten years ago, as illustrated in Figure 1. The total proposed supplement of $\$ 150 \mathrm{M}$ spread over three years is but one-and-ahalf percent of the projected cost of the planned SSC project, but its importance to the U.S. program is enormous ${ }^{3}$. Just how indispensable it is becomes evident when we consider next the implications, and the lost scientific opportunities, if there is no such supplement.

\section{Budget Scenario B: a flat budget}

This funding profile assumes that the high-energy physics program remains at the FY1995 level in constant-value dollars without the three-year bump included in Scenario $A$. In the above discussion, we emphasized that the main purpose of the temporary budget supplement is to restore the vitality of the existing program. Involvement in the LHC is made possible by the availability of funds freed up in FY1999 following the completion of the two major upgrades and the planned reassignment of AGS operations to the nuclear physics program. It clearly follows, therefore, that the main consequence of no supplement would be continued damage to the current U.S.

\footnotetext{
${ }^{3}$ We note for comparison that the annual budget of CERN is roughly equal to the total annual budget of the U.S. high-energy physics program, and the total budget for high-energy physics in Europe is about twice that of the United States.
} 
program, already weakened by the $7 \%$ loss in funds for operations and equipment in each of the last three years.

If there is no possibility of a three-year funding supplement to restore the national program in the period FY1996 to FY1998, the subpanel recommends that the Department of Energy appoint a special subpanel of the High Energy Physics Advisory Panel (HEPAP) to review the current U.S. high-energy physics program (preferably in cooperation with the National Science Foundation and including research supported by both the National Science Foundation and by the Department of Energy) and to recommend appropriate changes and reductions in scope. We do not believe that this problem can be addressed by continued proportional budget decreases at each of the laboratories and in each area of the program. We do believe that new priorities would have to be set that would likely call for sacrificing important parts of the U.S. program in order to preserve quality and productivity in what survives. The inevitable consequences will be continued loss of vitality in the current program and further discouragement to the new generation entering the field.

Beyond 1999, after the completion of the SLAC and Fermilab upgrades and the transfer of the AGS to the nuclear physics program, there would still be funds available in this budget scenario for participating in the LHC, as we discussed above in budget scenario $A$. (This is the same as illustrated in Figure 3 after the bump.) The overall level of participation in the LHC collaboration would depend upon the state of the national program at that point. In view of the importance of conducting research on the highest energy frontier, the subpanel recommends that the U.S. should still make a commitment to join the LHC, even if the budget remains flat at the FY1995 level of effort.

To join the LHC under a flat budget would require sacrifices to be made with respect to both U.S. involvement in the LHC and the rest of the U.S. particle physics program. The latter would have less diversity and less flexibility to implement new ideas. This would reduce the attractiveness of particle physics to the best young students looking toward scientific careers. The subpanel emphasizes that evidence of the U.S. government's sustained commitment to support the high-energy physics program is crucial to convince the best people who are drawn to the field that there will be exciting career opportunities for them in the future. The conclusion that a flat total budget will mean a 
serious loss in the vitality of the ongoing program is independent of participation in the LHC. Simply stated, a $\$ 50 \mathrm{M} /$ year shortfall under the flat budget scenario is serious and remains serious, whether or not a small initial commitment is made to the collaboration at the LHC at a level of roughly $\$ 5 \mathrm{M} /$ year. The significant start of U.S. participation in the LHC program would inevitably be delayed, as would the build-up of financial support. The inevitable consequence would be a loss of momentum in the collaborations that have been reforming to make use of the expertise and technology developed for the SSC. The level of involvement in the LHC program might be less than that indicated in the budget scenario in the previous section, which was, in our judgment, already on the lean side of optimal.

To summarize, it is the subpanel's recommendation that, in view of the importance of working at the highest energy frontier available during the next decade, and the importance of moving toward international collaboration in the twenty-first century, the government should support, and enter into negotiations for U.S. participation in, the LHC project under the flat budget scenario. Such a constrained budget will necessitate a delay in making any sizable contribution of funds in support of the LHC collaboration, but the door to U.S. involvement should not be closed. The key to this recommendation is retaining constant buying power in the long-term budget. This should allow the flexibility, following completion of the upgrades, to support the ongoing U.S. program and pursue advanced accelerator research and development toward future facilities, while making a significant contribution to the LHC project. 
(BLANK) 


\section{THE IMPORTANCE OF INTERNATIONAL COLLABORATION}

\section{A. INTRODUCTION}

Science is a truly universal endeavor. The questions, the methods, and the language of science transcend differences of politics, race, and culture. As Anton Chekhov wrote, "There is no national science, just as there is no national multiplication table; what is national is no longer science." Without the free exchange of ideas across borders, scientific progress would be slower, less fruitful, and needlessly wasteful.

High-energy physics has a proud history of international collaboration going back more than half a century. As the instruments required to work at the frontiers of the field grew in complexity and cost, it was natural for scientists in different countries to share ideas, tasks, and resources. Today, when the capability to do forefront research exists in only a few places around the globe, high-energy physicists routinely work hundreds or thousands of miles from home. The tradition of opening large accelerator laboratories to all qualified scientists, independent of their citizenship, was formalized some 15 years ago in guidelines set forth by the International Committee on Future Accelerators (ICFA); see Appendix $\mathrm{H}$.

As the scientific agenda drives the scale and cost of new facilities even higher, it is clear that worldwide progress depends more than ever on extensive international collaboration. We are entering an era where multinational plans have to be made for design, funding, construction, operation, and use of large accelerators. History has shown that such collaboration leads not only to better scientific understanding, but also to a better grasp of foreign viewpoints and values. International cooperation in science can pave the way to stronger partnerships between the nations involved.

We expect that the next few years will see extensive discussions among representatives of different governments about the best framework for such collaborations on international accelerators. We suggest some general principles to guide these negotiations: collaboration on research and development and design by interested parties, jointly agreed upon procedures for site selection, joint funding of construction and operation, common governance, and equal rights to all the benefits, such as industrial contracts, employment opportunities, 
and scientific use. For those issues to which they are applicable, ICFA guidelines should be followed. Of particular importance is that countries that do not join in construction of these accelerators should not be precluded from participating in research.

Imagination and courage in both science and politics will enable us to continue to address the fascinating and important questions posed by science that lie outside the reach of a single nation. The subpanel encourages the U.S. government to seek to enhance our country's ability to enter into international agreements on large projects in all areas of science where doing so would enable important questions to be addressed.

\section{B. HISTORICAL BACKGROUND OF INTERNATIONAL COLLABORATION IN HIGH-ENERGY PHYSICS}

Shortly after World War II, the U.S. became the undisputed world leader in particle physics. Scientists from around the globe came to do research at our preeminent laboratories. Many in an entire generation of Japanese and European leaders of the field received at least a part of their training in the U.S. Another popular route to international collaboration in the early days involved sharing of primary data: emulsions or bubble chamber film, exposed at U.S. accelerators, were then shipped to scientists abroad for analysis.

In the 1960s, as the experiments continued to grow in size and complexity, their performance required collaboration by groups from many institutions. These collaborations frequently cut across national lines, the criteria for collaboration being common interest in a problem rather than the common color of one's passport. As Europe, the Soviet Union, Canada, Japan, and China built their own accelerator laboratories, U.S. groups began to take advantage of unique opportunities abroad.

The level of these international collaborations was significant, both in terms of intellectual input and in terms of financial contributions to the experiment. For example, groups from Italy and Japan were partners in the design and construction of the Collider Detector at Fermilab (CDF) and contributed about one-third of the effort and of the total cost of about $\$ 100 \mathrm{M}$. In turn, U.S. scientists have been and are involved in the design, construction, and operation of the ZEUS detector at the HadronElektron-Ring-Anlage (HERA) collider at the Deutsches ElektronenSynchrotron (DESY) in Germany; several detectors at the Large Electron Positron (LEP) collider at the European Laboratory for Particle Physics 
(CERN); the AMY detector at the TRISTAN collider in Japan; and the MACRO detector in the Gran Sasso Laboratory in Italy. The U.S. contribution to ZEUS, built in the late 1980s and early 1990s, was roughly $\$ 25 \mathrm{M}$ and to the L3 detector at the LEP collider, constructed in 1983-1989, about \$50M.

These collaborations were initiated, formed, and executed almost entirely by scientists working together to achieve a common goal. Sometimes they operated within a framework of bilateral national agreements, but on the whole there were few government-level directives. Without a doubt, they have been remarkably successful. The sharing of talents and resources led to scientific productivity and improved cultural understanding. Scientists from nations that were enemies in World War II worked together right after the war. Even at the height of the Cold War, productive experimental collaborations between scientists from the U.S. and the Soviet Union took place in both countries.

Future efforts to advance the high-energy frontier will almost certainly be pursued within a framework of multi-national or international collaboration. Such collaborations will be necessary to advance both the hadron and electron frontiers. For the U.S. to be successful in this arena, new ground has to be broken in two areas: we have to go beyond collaboration on detectors and on data taking and analysis to collaboration on design and construction of accelerators, and, because of the required scale of these efforts, the U.S. government must be involved.

International collaborations to build accelerators have been quite successful. An outstanding example is CERN, an organization founded in 1954 to pool the resources of Western European countries to conduct high-energy physics research. The record of that institution in construction and operation is outstanding, and CERN today is one of the foremost high-energy physics laboratories in the world. With the recent admission of four Eastern European states, nineteen countries are CERN member states. Another excellent example of international collaboration in accelerator construction is the HERA collider at DESY, built by Germany with significant participation of several other countries. 


\section{Steps TOWARDS INTERNATIONAL COLLABORATION ON LARGE SCIENCE PROJECTS}

A growing number of scientific disciplines will soon require large collaborations or laboratories to address salient issues. Nuclear fusion, deep ocean drilling, and studies of the human genome are examples of such research.

International collaboration in scientific research requires understanding and confronting complex motivational and strategic issues. Long-term benefits and gains must be recognized and defended against short-sighted forces. Agreements between nations must be stable over time and different governing administrations. Above all, nations must have confidence in themselves and their partners. We do not possess the keys to such issues, but as participants in a scientific field that historically has gained much from collaboration across national boundaries, we offer a number of observations:

Any successful research collaboration must have scientific leadership and be driven by scientific needs and interests. There must be extensive scientific participation in research and development and design of the facility from the very beginning. (Research and development for electron-positron linear colliders being carried out currently under a multi-national memorandum of understanding is a good example of such early collaboration on the scientific level.)

The U.S. must establish anew its credibility as a reliable international partner and must be prepared to undertake and honor long-term commitments. (For this, we offer specific suggestions in chapter $\mathrm{X}$.)

The Department of Energy and the National Science Foundation are the natural governmental agencies to play a lead role in negotiation and management of collaborations in particle physics. They must be able to deal not only with foreign governments but also with international organizations, such as CERN, which represents many of these governments in the area of high-energy physics. In conjunction with the appropriate members of the U.S. high-energy physics community, these agencies are best qualified to shape specific collaborations to ensure that they fully take into account the nation's strengths and needs in scientific, technical, and industrial areas. The internal structures of these agencies must be organized so as to be optimally positioned for such negotiations. 
Close cooperation with the Office of Science and Technology Policy in the White House and with the State and Commerce Departments is essential. Strong support from the White House itself is necessary so that such collaborations can be raised to the negotiating agenda when leaders of different nations meet. Such high-level intervention has been, and will continue to be, essential in negotiating cooperation in large technological projects, both inside and outside high-energy physics.

\section{U.S. PARTICIPATION IN THE LARGE HADRON COLLIDER}

Participation by U.S. scientists and industry in the design, construction, and use of the Large Hadron Collider (LHC) at CERN would continue the international tradition of high-energy physics and extend the U.S. presence in global large science projects. This step would require careful thought and negotiation about the level and structure of U.S. involvement. Here are several guidelines:

When we construct principles for entering into such international agreements, we must place our scientific vision at the core. In negotiating for U.S. participation in the construction of the LHC, the U.S. should aim to extend to accelerators the praxis of international collaboration that has been used in building detectors and performing experiments as incorporated in the ICFA guidelines. Such an agreement might follow the pattern used at DESY to involve foreign countries in the construction of HERA. There, the most common form of participation was through the contribution of technical components built in the donor country. For example, about half the dipole magnets were built by industry in Italy. Other countries contributed scientific and technical personnel who played a significant role in the construction of the machine. Subsequent international participation in conducting experiments at HERA requires a contribution to cover only an appropriate share of the costs of running the experimental equipment, not the accelerator.

An agreement founded on similar principles would provide a realistic basis for U.S.-CERN collaboration, both for the construction of the LHC and for its subsequent scientific use.

At the end of construction of the LHC, or at any moment before, the U.S. might wish to participate actively in the decision-making process inside CERN. An appropriate structure, such as associate membership, could then be developed with CERN that would allow this participation. This issue, should it arise, can be discussed separately. It might involve contribution to the CERN operating budget. It might also be an 
important step toward acquiring experience about the optimum way for the U.S. to participate in future international construction of large scientific facilities. 


\section{TOWARD IMPROVED MANAGEMENT OF THE PROGRAM}

\section{A. STEPS TOWARD GREATER EFFICIENCY}

The potential for the U.S. to make historic contributions in highenergy physics must be realized in a way that uses the public's money and other resources as efficiently as possible. To ensure that this goal is achieved, we have identified a number of aspects of the program that should be addressed by the Department of Energy in collaboration with the high-energy physics community.

1. Review the Scientific Program. It is timely for the Department of Energy and the high-energy physics community to examine whether the high-energy physics program is being pursued at the highest scientific level possible with the funding available. Issues that should be addressed include the relative scientific performance of the highenergy physics groups in universities and in Department of Energy laboratories, whether savings are possible through consolidation or staging of related experimental programs, and whether the balance between the laboratory and university research programs is optimal. In addition, it is important to determine whether sufficient resources are being allocated to the research of young scientists with new ideas and initiatives.

Diversity, competition, and alternative approaches to common scientific goals are necessary for maintaining the strength of the U.S. high-energy physics program. However, it is wasteful to duplicate instruments and experiments without strong scientific and technical arguments. Plans must be carefully coordinated, particularly among the national laboratories. Large projects should be scheduled so as to avoid unproductive competition for limited resources and excessive pressures to reduce operating budgets to support construction.

The subpanel has not been in a position to focus on these issues, but concerns are widespread and they warrant a serious review, which will be a large undertaking.

As a first step, the Department of Energy Division of High Energy Physics, with suitable input from the National Science Foundation, the High Energy Physics Advisory Panel (HEPAP), and the American Physical Society's Division of Particles and Fields (DPF), should consider the scope and means of implementing a broad comparative assessment of these issues. Depending on the outcome of those considerations, a further in-depth study may be warranted. 
2. Establish Predictable Funding. High-energy physics projects are large and costly multi-year commitments. In recent history, they generally have been funded through the appropriations process, without prior Congressional authorization. The subpanel emphasizes the importance of future major high-energy physics construction projects being fully authorized at the start of the project. This process, although it does not guarantee full funding, can be important in building the support in Congress that is essential for success of a large project. It can also help ensure that projects proceed efficiently and expeditiously.

The importance of multi-year funding has been emphasized by the Carnegie Commission on Science, Technology, and Government in its 1994 Report "Science, Technology and Congress: Organizational and Procedural Reforms" (page 20). "Congress should extend program and project funding cycles for Science and Technology programs by adopting a variety of multi-year funding mechanisms, such as multi-year appropriations, advanced or forward funding, and up-front funding for major construction projects. All such programs should adopt standardized granting and accounting procedures." This recommendation is particularly relevant for the high-energy physics program because of the crucial role of large and expensive accelerators and detectors, and of long-term collaborations.

High-energy physics programs are currently hampered by legislative "fire-walls" between accounts for physics research, facility operations, technology research and development, and capital equipment. Providing Department of Energy Program Officers and laboratory directors with significant flexibility in moving funds from one account to another would improve efficiency by allowing them to be more responsive to new programmatic needs. The current severe limitations in being able to carry over operating funds before the end of each fiscal year also reduce efficiency. Often it is necessary to choose between spending money prematurely and losing it entirely. This situation can lead to designs being frozen before the best has been achieved, and to the purchase of less-thanoptimal components and equipment. Department of Energy program officers and laboratory directors should be given more flexibility to carry forward some fraction of total funds from year to year.

Another important aspect of funding concerns research that crosses the boundaries of several disciplines. We encourage efforts to insure that excellent projects, in particle astrophysics in particular, receive 
appropriate priority through broad multidisciplinary reviews, and sharing of the support when appropriate between disciplinary units within the funding agencies.

3. Overcome Excessive Administrative Burdens. Broad concerns were expressed to us from throughout the high-energy physics community about the growing administrative burden, beyond what is needed for adequate and responsible accountability. Strong criticisms were also voiced of what is perceived to be an excessively bureaucratic and inherently inefficient approach toward achieving full compliance with Environmental, Safety, and Health (ES\&H) regulations. The intention of these regulations-to promote operations that are safe for people onsite, the surrounding communities, and the environment-cannot be quarreled with. The problem is with the procedures for applying regulations that are not risk-based and that have not been evaluated for their contribution to safety.

The associated costs of meeting all of these requirements represent a significant fraction of the cost of running the Department of Energy's high-energy physics laboratories. In 1993, a direct official comparison of the cost of building a major project at a Department of Energy laboratory and at a National Science Foundation laboratory revealed an immediate up-front added cost of approximately $7 \%$ for the Department of Energy proposal, due to ES\&H regulations and documentation for quality assessment and program management (both proposals satisfied the same Federal regulations). Inefficient procedures for applying ES\&H regulations can cause a substantial loss of productivity. At Fermi National Accelerator Laboratory (Fermilab), during the past five years, ES\&H costs grew from $5 \%$ of the operating budget to the present figure of $10.8 \%$. In FY1994, the Stanford Linear Accelerator Center (SLAC) will allocate 13\% of its operating funds to ES\&H. The ES\&H costs at the Alternating Gradient Synchrotron (AGS) accelerator at Brookhaven National Laboratory (BNL) are of comparable percentage. In spite of the significant escalation of ES\&H costs, there has been no assessment of their overall benefit in terms of ES\&H goals.

The President has emphasized the need for efficiency in all federal agencies. We believe that it is urgent for the Department of Energy to seriously examine the cost effectiveness of its approach to applying its policies and procedures for assuring accountability and 
ES\&H compliance. This includes a reassessment of the cost/benefit value of the numerous reviews of the national laboratories; for instance, at BNL in FY1993, a total of 146 non-scientific reviews and 230 program reviews were conducted, requiring 4,851 person days.

\section{B. IMPROVING GOVERNANCE}

Concerns related to the governance of the U.S. high-energy physics program are much on the minds of the community, as reflected in many of the letters received by the subpanel. The subpanel agrees that ensuring enlightened and effective governance is essential if our vision of high-energy physics for the future is to be realized.

1. Organization of Particle Physics in the Department of Energy. In the Office of Energy Research, the Director of the Division of High Energy Physics and, in parallel, the Director of the Division of Nuclear Physics report to the Associate Director of the Office of Energy Research for High Energy and Nuclear Physics. The Associate Director, in turn, reports to the Director of the Office of Energy Research. Authority for program decisions rests with the Director of the Division of High Energy Physics and higher level officials in the Department of Energy.

The High Energy Physics Advisory Panel (HEPAP), an advisory board that has existed for more than 25 years, also reports to the Director of the Office of Energy Research. This panel is made up of physicists from the national laboratories and universities.

Traditionally, HEPAP has advised the Department of Energy on the overall quality of the research program as well as on how to balance initiatives for the future with a strong and diverse current research program within a given budget. In past years, before the Advisory Committee in Government Act (the so-called Sunshine Law), HEPAP was able to work in closed session, and even advised the Department of Energy during preparation of the program budget for a given fiscal year before it was announced. More recently, HEPAP has formed ad hoc subpanels that can work in private to recommend priorities and to review specific new initiatives and proposals in accord with budget guidance given by the Department of Energy. Since 1988 there have been five such subpanels dealing with broad priority issues: the Treiman subpanel (1988), the Wojcicki subpanel (1989), the Sciulli subpanel (1990), the Witherell subpanel (1992), and the present subpanel. 
While HEPAP is the only national advisory panel, there are a number of additional advisory bodies, some standing and others ad hoc, that provide advice on individual elements of the high-energy physics program. They include the Scientific Policy Committees and Experimental Program Advisory Committees at the individual laboratories. It is important to note, however, that these mechanisms for reviewing individual laboratory programs and university research groups operate on a case-by-case basis. They do not compare different laboratories or different university groups.

2. Community Concerns. In our discussions with high-energy physicists, in statements made at town meetings, and in letters we received from some 400 members of the high-energy physics community, the subpanel has heard numerous comments on perceived weaknesses in the governance of the U.S. high-energy physics program. These comments have addressed structural and institutional issues, as well as HEPAP, the Department of Energy, the directors of the national high-energy physics laboratories, and others.

Some of the concerns that have been expressed may be interpreted as natural reactions to the great stress, disappointments, and uncertainties generated in the high-energy physics community due to the cancellation of the Superconducting Super Collider (SSC) and decreases in research support in recent years. Nevertheless, legitimate issues have been raised that deserve timely consideration. In addition, we anticipate new modes of conducting research and development with a greater emphasis on international collaboration. Furthermore the escalating costs of individual experiments and accelerators mandate even greater care in planning so as to avoid any unnecessary duplication of effort.

Given these circumstances, the subpanel believes a thorough review of governance of the field is in order and should be undertaken by the supporting government agencies, the Department of Energy and the National Science Foundation, in cooperation with the community through the American Physical Society's Division of Particles and Fields. Not only our responsibility to use public resources wisely, but our desire to make significant scientific progress, dictates that we support research and training of the highest quality and ensure that facilities and equipment are adequate to achieve our scientific goals. Without comparing the various laboratory and university undertakings with each other, we cannot be sure that resources are being allocated optimally. 
Following our own discussions and communications with the community, the subpanel wishes to highlight three issues:

a. There are four high-energy accelerator laboratories in the U.S., three funded by the Department of Energy (Fermilab, SLAC, and BNL) and one by the National Science Foundation (CESR at Cornell). These have nurtured a diverse and vital program of experiments attacking critical issues in high-energy physics. But, as budgets have become tight, the need for coordination and cooperation between the laboratories has increased. When new instruments or upgrades are proposed, or when large experiments at different laboratories are

proposed for the same time frame, it is important that priorities be established broadly and in the context of the full national and international high-energy physics enterprise.

b. The university program does not have the same level of advocacy within the system as do the national laboratories. National laboratories are represented by strong directors who effectively advance the interests of their laboratories within the Department of Energy and are also capable of advancing their causes in the political arena. University groups, on the other hand, have no comparably visible advocate to represent their interests to the Department of Energy and to the National Science Foundation when they diverge from the interests of the national laboratories.

c. HEPAP, as the only standing national advisory panel for highenergy physics, needs to be a more effective voice for the high-energy physics community, especially in establishing national scientific and programmatic priorities. HEPAP must represent the major segments of the high-energy physics community in a balanced way.

\section{Suggested Remedies}

a. To help clarify, establish, and regularly review priorities for the field, a HEPAP subpanel should be convened every two years and charged with broadly reviewing the national program. It would also be within the purview of such 
subpanels to study special issues of relevance to the health of the U.S. high-energy physics program, as in the past. The subpanels should have significant overlap in membership, to foster continuity and to provide institutional memory.

Further, because the National Science Foundation plays a significant role in funding U.S. High Energy Physics, the subpanel supports an arrangement strengthening and formalizing the advisory role of HEPAP to the National Science Foundation, without attempting to restrict the independence of the National Science Foundation's decisionmaking .

Broad community input in the selection of HEPAP membership is essential.

b. The Director of the Division of High Energy Physics should solicit broad input from the high-energy physics community early in the budget planning process. While laboratory directors have a large role to play, special effort should be made to ensure that theirs are not the only voices heard. One approach would be for the Director of the Division of High Energy Physics to conduct each year, early in the budget planning process, at least one meeting of all laboratory directors and several well-regarded researchers from the universities. The meeting should focus on a discussion of budget proposals and laboratory programs in the light of national scientific priorities. Participants in these meetings would not be constituted as an advisory board, nor would they be asked to reach a formal consensus or write a report. Therefore, such meetings could be held in private, but with no prohibitions on the individuals expressing their views publicly. An advantage of involving such groups early in the setting of priorities and the planning of budgets is that all large budget requests from laboratories and large collaborations would come under the scrutiny of other laboratory directors and of well-informed members of the high-energy physics community. This could contribute to better and broader understanding and more rigorous justification of program decisions.

c. The bonds between the high-energy physics community and the Department of Energy's Division of High Energy Physics should be further strengthened at the operational level. It is 
especially important that there is a good mutual understanding of community concerns and funding issues as the high-energy physics program adjusts to the loss of the SSC and reductions in the budget. Most directly, this understanding can be improved by expanding the current procedure of appointing respected working members of the community to temporary positions in the Division of High Energy Physics for one or two years. The Director of the Division of High Energy Physics, with the assistance of the American Physical Society's Division of Particles and Fields, should identify knowledgeable, active high-energy physicists and give them program-wide responsibilities.

d. The subpanel reached consensus on the desirability of regular, substantive reviews of each laboratory director, conducted every five years or so by the contracting organization. Such reviews should ensure community input through membership on the review committee. 


\section{USING SUPERCONDUCTING SUPER COLLIDER ASSETS}

On behalf of the entire high-energy physics community, the subpanel wishes to acknowledge the extraordinary generosity of the State and the people of Texas in connection with the Superconducting Super Collider (SSC). Large contributions from a state toward the construction of a scientific laboratory are rare; as far as we know, they are unprecedented on the scale of the more than \$1B Texas pledged to the SSC. Furthermore, Texas did not limit its contributions to construction and equipment located within its state lines: through the Texas National Research Laboratory Commission (TNRLC), the State provided funding for SSC-related research and development to university groups across the country. In addition to financially demonstrating its awareness of the importance of high-energy physics, Texas was an outstanding host to the people who came to work on the SSC from across the country and around the world.

Although world science has now lost the SSC, it is vital to remember that not everything that went into the SSC has also been lost. Both intellectual and physical assets remain. The research and development that were carried out are still available to science and industry, through the literature and through the experience of many excellent scientists and engineers. Most of these people are now-or will soon be-applying their knowledge and experience in industry and in government laboratories, or engaged in high-energy physics research and teaching in universities or at the high-energy physics laboratories. The physical assets consist primarily of equipment on the Ellis County site.

Of the approximately $\$ 2 \mathrm{~B}$ expended on the SSC to date, the largest component was in salaries of scientific, technical, and support personnel, both on-site and off-site. About $\$ 135 \mathrm{M}$ was invested in movable technical equipment including computers and is in place at the site. Approximately $\$ 100 \mathrm{M}$ was invested in buildings and utilities and other non-movable but reusable assets.

The resources in place at the SSC were superb and admirably suited to their intended purpose. The Central Facility, Magnet Development Laboratory, and Magnet Test Laboratory are large, modern buildings housing state-of-theart tools to create and test superconducting accelerator magnet prototypes for the SSC, as well as to perform subsequent quality control of magnets produced in industry. A large helium refrigeration system exists to support this work. Other support facilities, such as machine shops, are excellent. The construction of the linac, the first accelerator in the SSC complex, was well under way. A powerful array of distributed computers was assembled. 
The subpanel understands that a vigorous process for encouraging and reviewing proposals for on-site use of major facilities is now under way under a cooperative agreement between the Department of Energy and the TNRLC. There are many possibilities: educational, medical, scientific, and commercial-or some combination of these. The subpanel applauds this effort to make good use of the on-site investment. We wish to stress that if a scientific mission is contemplated, proposals should undergo a stringent peerreview process. The process should call upon experts in relevant areas of science chosen from the national and international communities, and proposals should be judged on scientific and technical merit, feasibility, and costeffectiveness in comparison to other possible avenues for conducting the same activity.

One possible use of the equipment on the site is a superconducting magnet laboratory that would be the center for U.S. participation in the European Laboratory for Particle Physics LHC project. If such a proposal is brought forward, it too should be evaluated as described.

The subpanel anticipates difficulties with a superconducting magnet laboratory for high-energy physics at the former SSC site. The powerful cadre of scientists and engineers that came together to design, assemble, and operate the SSC has now been widely dispersed. Prospects for rebuilding and maintaining a scientific and technical staff of the highest caliber in relative isolation from the high-energy physics enterprise do not appear to be good. Many of the principals in superconducting magnet development in the U.S. are particle physicists who view their work as part and parcel of doing particle physics. Superconducting magnets are components of a complete instrument, consisting of an accelerator and detectors together. The design of one depends on the other and requires continual and easy communication, knowledge transfer, and compromise. Such would have been the case at the SSC. However, a high-energy physics magnet laboratory geographically separated from an accelerator laboratory will not be able to provide this environment.

Another potential issue is whether maintaining such a facility would be cost-effective in the long run. While short-term savings from using existing equipment on site may be possible, the cost over time of maintaining the administrative and technical staff needed to support these activities may be larger than at one of the existing high-energy laboratories, where staff resources and other infrastructure can be shared with other high-energy physics activities. 
These concerns will have to be weighed in evaluating proposals that may be brought forward for a superconducting magnet laboratory for high-energy physics on the former SSC site. The subpanel believes that they weigh against a decision for such use.

Disposition of movable equipment will depend upon agreements between Department of Energy and the State of Texas, which may involve the resolution of financial claims of the State. The subpanel does not presume to comment on issues outside our scientific and technical expertise in high-energy physics. If equipment will not ultimately be used on site, it could be used effectively elsewhere to the benefit of the U.S. high-energy physics program. In the event that movable equipment may ultimately be deemed to be the property of Department of Energy and relocatable, the subpanels wishes to provide guidelines for its disposition.

a. Equipment directly related to the operation of accelerators or to the development and fabrication of magnets and large detector facilities should be relocated to national high-energy physics laboratories where it can be integrated into ongoing activities. Large-scale systems that can strengthen ongoing laboratory activities should also be relocated to these sites.

b. In those cases where equipment is of such a scale and nature as to be useful in university-based high-energy physics research, detector development or fabrication, or accelerator research, preference for relocation should be given to university-based high-energy physics groups.

The progressive decline of university infrastructure has been a serious concern for several years; the 1988 High Energy Physics Advisory Panel (HEPAP) subpanel on Future Modes of Experimental Research in High Energy Physics explicitly identified this as a serious problem. This decline has continued with only some mitigation, ironically, as the result of research grants to universities from TNRLC that were often used to improve university infrastructure. Equipment at the SSC site is modern and of high quality. The subpanel believes the transfer of equipment to university-based groups can help to address the infrastructure problem.

Finally, the subpanel would urge all parties to decisions regarding the disposition of SSC assets to recognize that a timely resolution of the issues will make it more likely that they will be put to good use. 
Appendix A 
This appendix is a chronology of activities and decisions that led to the creation of the Superconducting Super Collider (SSC) project, and of its subsequent progress and accomplishments.

The interests of the high-energy physics community in a multi-TeV accelerator began to take shape in a series of International Committee on Future Accelerators (ICFA) workshops in 1978 and 1979, where a proton-proton collider with an energy of $20 \mathrm{TeV}$ per beam was first discussed. The SSC project itself had its origins in the 1982 Snowmass Summer Study sponsored by the Division of Particles and Fields of the American Physical Society. Several other workshops, including two major ones at Cornell and Lawrence Berkeley Laboratory (LBL) on accelerator and detector technologies respectively, then provided the basis for the recommendation by the High Energy Physics Advisory Panel (HEPAP) in 1983 for "immediate initiation of a multi-TeV high-luminosity proton-proton collider project with the goal of physics experiments at this facility at the earliest possible date." This large leap forward in the scale of accelerator technology was agreed to be necessary to elucidate the physics of electroweak symmetry breaking, and hence necessary for continued progress in high-energy physics.

As a result of the HEPAP report, formal research and development support for the SSC was initiated in fall 1983, and the Department of Energy and the directors of the U.S. high-energy physics laboratories chartered a series of preliminary studies for the SSC. Thus began the National Reference Designs Study, started in December 1983, to study the technical and economic feasibility of a machine with the designated parameters of $20 \mathrm{TeV}$ per beam and a luminosity of $10^{33} \mathrm{~cm}^{-2} \mathrm{sec}^{-1}$. By April 1984, these initial studies had been completed by a team of about 150 engineers and accelerator physicists. Three different reference designs were presented, based on three distinct types of superconducting magnets, all of which were deemed technically feasible. A preliminary cost estimate was produced for each of the designs.

The next step was the formation of the Central Design Group (CDG), based at LBL and managed by the Universities Research Association (URA) in summer 1984. This effort was directed by Professor Maury Tigner. In parallel, extensive work on prototype magnets was launched in several national laboratories--Brookhaven National Laboratory (BNL), Fermi National Accelerator Laboratory (Fermilab), and LBL, as well as the Texas Accelerator Center (TAC), studying five different designs. This effort led to the selection of a magnet design based on a single cold bore with a high field of 6.5 Tesla in 1985. Additional work on site specifications and a detailed site-independent cost estimate, as well as engineering refinements of the magnet design, led to a complete conceptual design for the project. In total, a group of roughly 250 scientists and engineers participated in the CDG and contributed to the Conceptual Design Report published in 1986. The SSC machine described in this report embodied many technical challenges. A broad-based accelerator research and 
development program, encompassing high-field superconducting magnets, vacuum and thermal problems associated with synchrotron radiation, beam dynamics, and energy losses had been initiated in 1984 under the CDG, and would proceed over the following decade to address these challenges. Major challenges also existed for the experimental program, and a detector research and development program, administered by the Department of Energy with assistance of the CDG, was started in 1987 and continued through 1992.

After extensive Department of Energy review, a Presidential decision to proceed with the SSC was made in January 1987 and a site selection process was initiated. A total of 43 proposals were received, 35 of which met the necessary guidelines. After examination by a committee assembled under the auspices of the National Academy of Sciences, seven proposals were selected for further Department of Energy review. The Ellis County, Texas site was announced as the preferred site by the Department of Energy in November 1988, leading to the creation of the SSC laboratory under the directorship of Professor Roy Schwitters, and the management of URA, in January 1989. A series of international advisory bodies were formed by the lab director, including the Scientific Policy Committee, the Program Advisory Committee, and the Machine Advisory Committee. The Texas National Research Laboratory Commission (TNRLC) was formed in 1988 to oversee the Texas interest in the SSC. Starting in 1990 , it created a program to distribute, based on extensive peer review, approximately $\$ 100 \mathrm{M}$ over a period of ten years to universities in support of SSCrelated research and development throughout the U.S.

One of the initial tasks of the laboratory was the creation of the site-specific conceptual design, completed in July 1990. As the site-specific design became more detailed, experience with the Hadron Elektron Ring Anlage (HERA) magnets, and simulations of the full $10^{7}$ turns required for injection, led to a decision to change several aspects of the original design toward a more conservative one. Changes were proposed and agreed upon, including increasing the main ring dipole aperture from $40 \mathrm{~mm}$ to $50 \mathrm{~mm}$ to improve operating margins and field quality, and increasing the injection energy from $1 \mathrm{TeV}$ to $2 \mathrm{TeV}$. Numerous technical experts agreed that these changes were essential for rapid commissioning and reliable operation of the accelerator. Detailed reviews of the energy and luminosity goals of the design were carried out by an Ad Hoc Committee and by a HEPAP subpanel. Both affirmed the design parameters of $20 \mathrm{TeV}$ per beam and a luminosity of $10^{33} \mathrm{~cm}^{-2} \mathrm{sec}^{-1}$. The sitespecific conceptual design, a basic construction plan, and a detailed cost estimate were then extensively reviewed by the Department of Energy Program Office as well as by the Department's Independent Cost Estimating staff, and the project cost and schedule baseline were established. As the site-specific design process was completed, the final footprint of the machine was delivered to the Department of Energy in December 1989, and in March 1990 the State of Texas began acquiring some 16,000 acres of land. 
The necessary Environmental Impact Statement was completed by the end of 1990, and was issued following the Record of Decision. First major construction at the SSC site began in 1991 at the N15 site, home of the Magnet Development Lab (MDL), the Magnet Test Lab (MTL), and the Accelerator Systems String Test (ASST) facilities. These facilities, upon completion, represented fully-equipped work areas of 200,000 square feet, capable of producing 25 magnets per year (needed for the various specialized magnets for the accelerator) and testing ten dipole magnets simultaneously. The superconducting magnet program, with the goal of producing $50 \mathrm{~mm}$ dipole magnets for the string test, was initially carried out by a collaboration among the existing laboratories (BNL, Fermilab, LBL). A total of 20 dipoles were produced, 13 at Fermilab and seven at BNL. These magnets were built in collaboration with staff from industrial partners: General Dynamics at Fermilab and Westinghouse at BNL. Six full-length prototype quadrupoles were built at $\mathrm{LBL}$, and an additional five by the industrial partner Babcock and Wilcox. All of these magnets performed well, satisfying the required operating margins and field quality. A first major milestone, the string test, involved the operation of a string of five dipoles and a quadrupole, the basic half-cell of the accelerator, in the ASST facility. This was completed in August 1992. It was followed by a second phase test with a full-cell of ten dipoles and two quadrupoles. Meanwhile, the MDL was building further prototype magnets, innovative work on corrector magnet technology was being done, and design and prototyping work for the very challenging final focus magnets was going ahead.

Detailed design and early construction work was proceeding on all major machine components. The conventional construction for the injection complex, consisting of the ion source and a linear accelerator stationed in a 250-meter tunnel, was complete and component installation was under way. The first circular accelerator in the chain, the Low Energy Booster (LEB), consisting of a 600-meter circumference ring filled with resistive magnets, was designed and $90 \%$ of the tunnel complete. The next element in the sequence, the Medium Energy Booster (MEB), consisting of a ring of 4.0 kilometers in circumference, again using resistive magnet technology, was designed and excavation of the tunnel had started. The third and final accelerator before entering the large collider rings, the High Energy Booster (HEB), consisting of 10.8 kilometer circumference tunnel filled with superconducting magnets, was under design. Finally, for the 87.1 kilometer circumference collider ring, the excavation of seventeen shafts was complete, and the tunnel boring, begun in January 1993, had proceeded rapidly, with 77,065 feet (roughly 23 kilometers) completed by fall 1993.

In parallel with the creation of the laboratory, the establishment of the experimental program for the SSC began with the call for Expressions of Interest in early 1990 . The international experimental community responded by submitting a total of 21 Expressions of Interest for experiments covering a wide range of topics. The initial experimental program was to consist of two large, general-purpose detectors and several smaller, more specialized experiments. Letters of Intent for the large experiments were prepared by November 1990, and the task of defining the 
experimental program proceeded. By late 1991, two large collaborations, GEM (formed in June 1991) and SDC (formed in September 1989), had converged on complementary detector concepts. After review of their Letters of Intent, both were approved to proceed with more detailed conceptual designs and to write Technical Design Reports. This led to the submission of the SDC Technical Design Report in April 1992, and the GEM Technical Design Report in April 1993. The SDC detector received Phase 1 Department of Energy approval in October 1992, and GEM was in the process of undergoing similar review in fall 1993. In total, a community of roughly 2,000 scientists and engineers from more than 200 institutions world-wide were involved in these two detector projects. A broad-based program of research, development and engineering, addressing instrumentation issues relevant for the SSC experimental program, was carried out over many years, producing advances in all areas of high-energy physics instrumentation. This provided confidence that the very ambitious experiments planned for the SSC could succeed.

Beyond the physics mission of the SSC, there was a program of educational outreach to high school students and teachers, colleges, and universities. The substantial investment in research and development for experimental instrumentation helped the ailing university high-energy physics infrastructure, in addition to the large number of significant improvements in detector technology that resulted.

Progress on the project was the fruit of many years of dedicated work and investment by many. A substantial number of scientists and engineers had relocated to Texas in order to construct this new facility. A total laboratory staff of over 2,000 employees, including more than 250 foreign scientists and engineers from 38 countries, was assembled. The SSC experimental program, which had broad international participation from the beginning, had benefitted from the substantial investment in SSC detector research and development. Operation at luminosities of $10^{33} \mathrm{~cm}^{2} \mathrm{sec}^{-1}$, which a decade before had seemed formidable, now was seen as entirely feasible for the major detectors detailed in the technical design reports, as well as for the collider itself. For both the accelerator and experimental systems, there were no technical show-stoppers when the project was terminated.

Everybody who worked to create the SSC can be proud of their very impressive technical achievements. 
Appendix B 


\section{The Secretary of Energy}

Washington, DC 20585

November 4, 1993

Professor Stanley Wojcicki

Chairman, High Energy Physics Advisory Panel

Department of Physics

Stanford University

Varian Building, Room 170

Stanford, California 94305

\section{Dear Professor Wojcicki:}

The termination of the Superconducting Super Collider (SSC) by the United States Congress raises major questions about the future of high energy physics research and about potential utilization of assets at the SSC site. The Department of Energy would like the High Energy Physics Advisory Panel (HEPAP) to turn its attention immediately to the task of defining a long-term program to pursue the most important high energy physics goals now that the SSC has been terminated. The information you provide will be helpful to the Administration as the SSC is terminated and plans are developed for the future of high energy physics research.

We are seeking a current assessment of the United States high energy physics program and recommendations for the future. Your assessment should consider the current and potential roles of all existing and approved facilities, and should provide priorities for funding in light of the termination of the SSC. Particular emphasis should be placed on options for establishing a truly intemational framework for construction, operation, and utilization of future high energy physics research facilities. This assessment should include recommendations of organizational structures for a program of expanded international collaboration that includes construction of large devices, development of detectors, and analysis of research data. We specifically would like recommendations of the practical steps necessary to facilitate enhanced international collaborations for the construction of large high energy research facilities in the future.

In connection with an examination of these issues, we would like recommendations on how to make the best use of the investment that has been made in the SSC. This should include a full exploration of options to perform worthwhile physics research using facilities developed as part of the SSC project. Finally, we look to HEPAP for recommendations on how to encourage displaced physicists and engineers to remain in the field and to attract young scientists to enter the field of high-energy physics in the future. 
The Department would like these issues to be addressed at the HEPAP meeting on November 8 and 9, 1993, and preliminary assessments provided immediately thereafter. In addition, we request that a dedicated subpanel be constituted to address these issues, with an initial report by February 28, 1994, and a final report by May 30, 1994. The recommendations of this report will be valuable in assisting the Department in meeting its requirement to provide Congress with a report by July 1 , 1994, on future options for high-energy physics research which the Department of Energy plans to support, and on utilization of assets at the SSC site.

I appreciate your prompt attention to this request.

Sincerely,

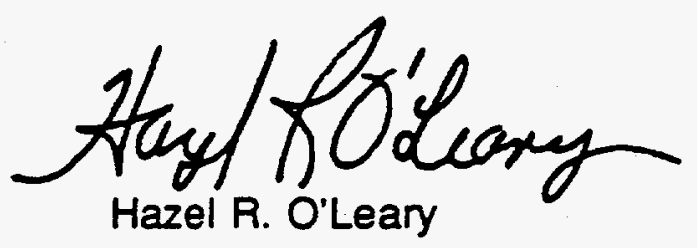


Appendix C 
High Energy Physics Advisory Panel

Subpanel on Vision for the Future of High-Energy Physics

Professor Sidney D. Drell, Chairman

Stanford Linear Accelerator Center

Professor Jonathan A. Bagger

Johns Hopkins University

Professor Patricia R. Burchat

University of California, Santa Cruz

Professor David L. Burke

Stanford Linear Accelerator Center

Dr. Joel N. Butler

Fermi National Accelerator Laboratory

Dr. Helen T. Edwards

Fermi National Accelerator Laboratory

Dr. Kevin Einsweiler

Lawrence Berkeley Laboratory

Dr. Lorenzo Foa

European Laboratory for Particle

Physics (CERN)

Professor Val L. Fitch

Princeton University

Professor John Huth

Harvard University

Professor Dan Kleppner

Massachusetts Institute of Technology

Dr. Akihiro Maki

Japan Society for the Promotion of

Science

Dr. William J. Marciano

Brookhaven National Laboratory
Professor Jack L. Ritchie

University of Texas at Austin

Professor Bernard Sadoulet

University of California, Berkeley

Professor Maury Tigner

Cornell University

Ex-Officio Members

Professor Roberto D. Peccei

University of California, Los Angeles

Past Chair, Executive Committee of the

Division of Particles and Fields,

American Physical Society

Professor Stanley G. Wojcicki

Stanford University

Chair, High Energy Physics Advisory Panel

Professor Michael E. Zeller

Yale University

Chair, Executive Committee of the

Division of Particles and Fields,

American Physical Society

Executive Secretary

Dr. Patrick D. Rapp

U.S. Department of Energy 


\section{Agency Observers}

Dr. Wilmot N. Hess

U.S. Department of Energy

Dr. John R. O'Fallon

U.S. Department of Energy

Dr. Robert Eisenstein

National Science Foundation

Dr. William Chinowsky

National Science Foundation 
Appendix D 
Stanford Linear Accelerator Center

P. O. Bax 4349

Stanford, CA 94309

(415) 926-2664

Telefax: (415) 926-4500

Bitnet Address:

BROSE at SLACVM

December 15, 1993

\section{Dear Colleagues:}

I am writing for your help. I have agreed to chair the HEPAP sub-panel being formed at the direction of Secretary Hazel O'Leary to build a new vision for the U.S. High Energy Physics Program now that the SSC is no more. Following my meetings in Washington with the Secretary and ER Director Dr. Martha Krebs, I am satisfied with the working assumption underlying this panel's work. It is that the U.S. HEP base program will be adequately supported to achieve its currently defined goal of completing the main injector at Fermilab and the B-Factory at SLAC circa 1998 while maintaining a healthy program of utilizing existing facilities. This will enable the new panel to focus on developing a vision of our field's future. I am enclosing a column from the Los Angeles Times of November 21 which sets out the challenge before us as seen by Secretary O'Leary and Congressman George Brown, Chairman of the House Committee on Science, Space and Technology. It is an excellent statement and its first and next to the last paragraphs are the challenge to the panel. We must clearly define and convey our scientific goals and a practical path to achieving them with international collaboration.

The new panel is now being assembled. We are committed to completing our work by May 25, 1994. I am inviting your thoughts whether conveyed by individual or group letters, E-mail or faxes. I assure you that we will give them serious consideration as we undertake our task.

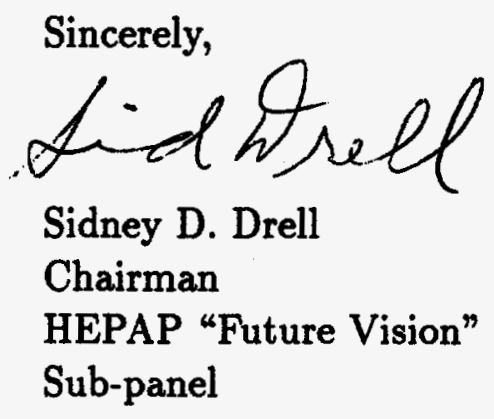

SDD:bgr

Enclosure 

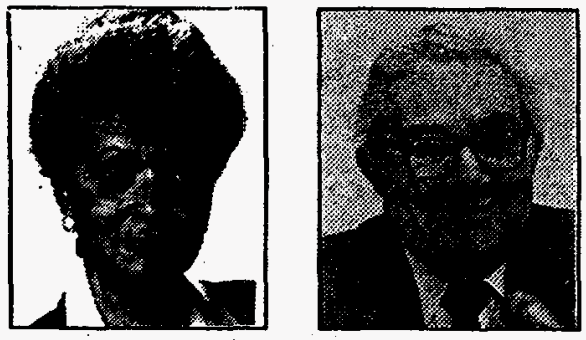

We called it international, but it wasn't. To succeed, big science projects must be fully multinational.

\section{BY HAZEL R. O'LEARY}

and GEORGE E. BROWN JR.

$\mathrm{T}$ The superconducting super collider as we know it is now dead, yet the quest for a comprehensive understanding of the world around us lives on. The scientific questions that compelled development of the SSC will not suddenly disappear, nor are they likely to be answered by anything other than a "big science" endeavor during the next century. For any such effort to succeed, however, this hypothetical future project-and perhaps all future big science projects - will need a level of international, political and public support that remained elusive for the super collider.

The SSC suffered for having failed from the outset to incorporate international funding and participation. The Reagan and Bush administrations made critical early decisions about the technical design and site location as if the SSC were purely a national project. Only later did they proclaim it to be an international collaboration - with a goal of nearly $\$ 2$ billion in foreign funding. Is it any wonder that substantial foreign funding never materialized? This shortfall eroded congressional support, which made foreign involvement even less likely, accelerating the project's downward spiral.

The obvious lesson to be learned is that foreign participation must be incorporated into large-scale science and technology projects from the very beginning; when prospective partners still have a say in why, where, when and how such projects will be pursued. Not so obvious is how we as a nation will make and keep such international agreements in the future.

Although the United States has determined that it cannot fund projects of this scale alone, neither have we demonstrated that we can undertake such endeavors with others. The abrupt termination of the super collider adds to a long list of large international projects that the United States has suddenly and unilaterally killed or drastically altered, including the Ulysses solar satellite program, the solvent-refined coal project and the space station. This embarrassing legacy raises serious questions about the reliability of the United States in international research projects.

Although Congress intensely criticized the super collider project for failing to receive substantial foreign funding, it was never clear that Congress was prepared to share with other nations the jobs and technological benefits that would have flowed from a true partnership. Is it realistic for the United States to want all the "good" jobs and all the critical technological components of a project like the SSC, while also insisting that other nations put billions of dollars on the table?

This raises a related concern: Political support for large projects appears to be directly proportional to the parochial benefits received, yet spreading the wealth of large scientific projects invites appropriate criticism of pork-barreling. When 25 states were competing for the SSC site, the level of political support was enormous. Elected officials nationwide-from senators to city supervisors-heralded the project as vital for the United States and also for their individual states. Once Texas was selected as the project site, however, this overwhelming interest vanished in a flash.

Such phenomena raise an extremely difficult issue for the future. Specifically, how can the nation stick with a decision that has scientific and technical merit before and after the potential economic benefits for individual regions of the country are determined? This issue is especially vexing for projects like the SSC, which require a long-term congressional commitment. It is further complicated both by the turnover of elected officials - which cripples institutional memory and commitments-and by the existing annual budget process, which encourages constant secondguessing of political decisions.

Finally, there is a lesson to be learned about public support for fundamental science. The super collider never captured broad support from the American public, in no small part because its scientific promise was difficult to understand even by those who are scientifically literate. As study after study has shown, science education in the United States lags far behind that of other industrialized nations. This suggests that a key to sustaining U.S. excellence in basic research will be aggressive efforts to improve scientific and technical literacy at every level of education.

With the help of a blue-ribbon panel on the future of high-energy physics, we are now putting the pieces back together from a project that blew apart after an extraordinary investment of human and national resources. The superconducting super collider held the promise of taking humanity to the next level of understanding about the origins of the universe and the fundamental dynamics of matter. Unless we are intent on stopping the pursuit of the knowledge that it would have delivered, we must find a way to achieve a truly international framework for large scientific and technological projects.

This will be the enduring challenge as the cranes and bulldozers in Waxahachie, Tex., come to a halt, as we attempt to soften the landing for SSC employees and as we look for the ways to continue the extraordinary journey of human inquiry that has brought us the scientific knowledge that underpins our society and fuels our economy.

Hazel R. OLeary is U.S. secretary of energy. Rep. George E. Brown JT. (DColton) is chairman of the House Committee on Science, Space and Technology. 


\title{
STANFORD UNIVERSITY
}

\author{
Stanford linear Accelmaton Center
}

\author{
P. O. Box 4 ine \\ Stenford, OA gestog \\ (418) $026-2064$ \\ thele: (415) 906-1500 \\ Bhtaet Addreas: \\ BROAR at BLAOVM
}

Fobruary 14, 1994

\section{Dear Colleagues:}

This is a follow-up to my December 15 letter to bring you up to date on the activities of the new "Future Visions Panel" formed to redefine the future of the U.S. High Energy Physice Program now that the SSC, our top priority commitmont of the part decade, has been cancelled. As I wrote in my first letter to you - "We must clearly define and convey our scientific goals and a practical path to achieving them with international collaboration." The Panel is hard at work. I sought broad advice in forming it and roceived masy excellent suggestions for Panel membership, partjcularly from the chairman of HEPAP and from the Division of Particles and Fielda. I believe that our community is admirably represented and served by the members selected. You have heard by now of planned town meetingt being organized in cooperation with the Division of Particles and Fields. I also remind you of my invitalion to you in the letter of December 15 to convey thoughts, concerns, recommendations, etc. to the Panel. I promised, and repeat, they will be given serious consideration.

I regret and an very disappointed that I have to make a correction to my December 15 letter to you. I wrote then that following discussions with the Secretary of Energy and the Director of Energy Research "I am satisfied with the working ssumption underlying this Panel's work. It is that the U.S. High Energy Physics base program will be adequately supported to achieve its currently defined goal of completing the Main Injector at Fermilab and the B-Factory at SLAC circa 1998 while maintajning a healtby program of utilizing existing fecilities. This will enable the new panel to focus on developing a vision of out field's future." The White House has now published the FY95 budget. It ia not what I believed it would be when I sent out that letter. In fact what it presents to us is a budget for the DOE High Energy Physics program in FYQs that will require further substantial contraction of the ongoing program, while it is trying to absorb scientists returning from the SBCL. Our current budget situation is as follows: The proposed (but not yet appropriated) budget for FY95 contains construction funds for the Main Injector and the B-Fuctory as scheduled. That is the good news. The bed news is that it also marks the third year in a 
row in which there is a decrease of more than $7 \%$ in constant dollars for support of operations and equipment in the ongoing program. There was also, in FY94, an additional lose of about $\$ 58 \mathrm{M}$, or $91 / 2 \%$, of resources that came to the community from SSCL and Texus funda in support of detectors and magnet research. Fortunately, that loss is, in part, offet by $\$ 14 \mathrm{M}$ of SSC closeout tunds that have been made available in FY94, but no such funds are provided in the budget presented for FY95.

With this budget the problems facing our field following the demise of the SSC are all that much more diffleult. Many of you have suggested that my Panel ahould recommend specific changes and new priorities in today's program in order to bring it in line with the current budget realities. While I agree with the urgency of this need $I$ atn writing to make clear that this is not the charge to the Panel. What the Panel will consider is the evolution of the current program as tcientific and fiuancial resources transfer to $R \& D$ in preparation for morking at the new frontiers. That is part of building a new vision and practical strategies for the field in the light of the loss of the SSC. This is what the Sectetary has asked of un and this is what I believe is urgently needed if we are to command national rupport for a warld competitive program in the next century.

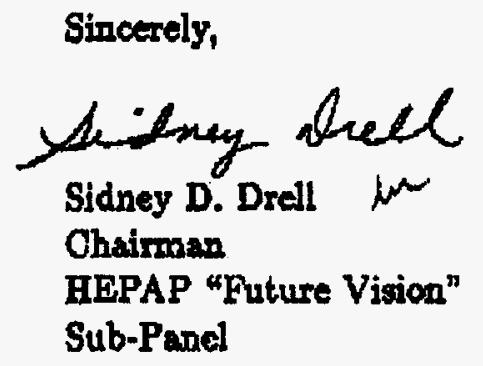

SDD:bgr 
To: Colleagues (THIS HAS GONE OUT TO THE COMMUNITY)

From: Sid Drell, Chairman

TOPIC: Future Vision Sub-panel of HEPAP

This is an update on the activities of the Sub-panel. I reported to HEPAP on March 7 and would like to send to you directly a sense of what our current thinking is. We still have a long way to go and are struggling mightily with the challenge to find the optimal balance between two essential components of a vision for the future of the U.S. high energy program. One to participate substantively in the effort to further advance the high energy frontier by continuing R\&D on magnets and detectors, including R\&D collaboration with CERN toward the LHC. The other essential component is to support good physics at existing facilities and at those currently being upgraded, namely the Main Injector, the B-Factory and CESR. This is required to sustain a strong university based program that can attract, train, and hold the best young researchers who are so critical to our field and its success. There is no one-two priority ordering of these components. One focuses on the decade ahead and sustaining a strong U.S. program among the world leaders at the available frontier. The other prepares for a longer teim future in search of answers to compelling questions at higher energies. Both are necessary and the Sub-panel is working to develop strategies towards a future program addressing both and, of course, restrained by budgetary "realities."

Your letters are important to the Sub-panel and have a very significant impact on our thinking. I am unable to respond substantively to each one individually, but let me here take the opportunity for a collective expression of appreciation to those of you, well over 100 in number, who have made the effort to express your concerns and present your recommendations to us. I am aware of the disappointment that has been expressed by some that more members of the Sub-panel did not attend the town meetings. You should be aware that we have heard and read substantive reports from those members who did attend. The typically four members present at each of these meetings have been very effective channels of communication from those meetings to the rest of us. We hear you.

SDD:bgr 
Appendix E 


Agenda
HEPAP Subpanel on Vision for the Future of High-Energy Physics
Stanford Linear Accelerator Center
SSRL Building
Stanford, California

Monday, January 10, 1994

1:00 pm Organizational Meeting

Executive Session

10:00 pm Adjourn

Tuesday, January 11, 1994

9:00 am

Organizational Meeting

Executive Session

10:00 am

Martha Krebs, Director

Office of Energy Research

11:30 am

Hazel R. O'Leary

Secretary of Energy

1:00 pm

Adjourn 


\section{AGENDA}

HEPAP Subpanel on Vision for the Future of High Energy Physics

Madison Hotel

Executive Chambers, 2nd Floor

15th \& M Sts, NW

Washington, D.C.

Sunday, February 6, 1994

9:00 am

Executive Session

9:30 am-6:00 pm Invited Briefers

C. Llewellyn-Smith, CERN

V. Soergel, DESY

T. D. Lee, Columbia University

B. Richter, SLAC

B. McDaniel, Cornell

W. Panofsky, SLAC

Break

K. Berkelman, Cornell

J. Peoples, Fermilab

N. Samios, BNL

J. Friedman, MIT

R. Schwitters, SSCL

J. O'Fallon, DOE

R. Eisenstein, NSF

$8: 00 \mathrm{pm}$

Executive Session 
Monday, February 7, 1994

9:00 am Executive Session

-Comments on Sunday's presentations

$10: 00$

$$
\begin{aligned}
& \text { Reports from Subpanel Working Groups } \\
& \text {-International collaboration } \\
& \text {-Accelerators } \\
& \text {-Theory } \\
& \text {-Experiment } \\
& \text {-Non-Accelerator } \\
& \text { Continuing Discussions } \\
& \text {-Report outline and assignments }
\end{aligned}
$$

$1: 30$

7:30 Continuing Discussions

Tuesday, February 8, 1994

8:00 am Continuing discussions

11:00 Adjourn 


\section{AGENDA}

HEPAP Subpanel on Vision for the Future of High Energy Physics

Radisson Suites Hotel/O'Hare

Ballrooms A \& B

5500 N. River Road

Rosemont, Illinois

\section{$\underline{\text { Saturday, March } 5}$}

9:00-10:00 am Executive Session; Reports from Town Meetings

10:00-11:30 am Report from LHC collaborations (G. Trilling, W. Willis, et. al.)

11:30-11:45 am Break

11:45-1:00 pm Americans working abroad: issues, opportunities

(Gail Hanson, Dave Stickland, Malcolm Derrick)

1:00-1:45 pm Lunch

1:45-2:30 pm Further input on non-accelerator frontier (Jim Cronin)

2:30-3:00 pm Beyond the LHC using Fermilab Main Injector (Bill Foster)

3:00-9:00 pm Executive Session

Sunday, March 6

9:00-6:00 pm Executive Session 


\section{AGENDA}

HEPAP Subpanel on Vision for the Future of High Energy Physics

Hanalei Hotel

San Diego, California

Saturday, April 9, 1994

8:30 am

Executive Session

9:30 am

Tom Nash

10:00 am

Barry Barish

10:30 am

Executive Session

Sunday, April 10, 1994

8:30 am

Executive Session

9:00 am

Ernie Moniz

9:45 am

Executive Session

Monday, April 11, 1994

Executive Session

Tuesday, April 12, 1994

Executive Session

Wednesday, April 13, 1994

Executive Session

Thursday, April 14, 1994

Executive Session 


\author{
Agenda \\ HEPAP Subpanel on Vision for the Future of High Energy Physics \\ Doubletree Hotel \\ 300 Army Navy Drive \\ Arlington, Virginia
}

Sunday, May 8, 1994

Executive Sessions

Monday, May 9, 1994

Executive Sessions

Tuesday, May 10, 1994

Executive Sessions 
Appendix F 


\author{
HEP TOWN MEETING \\ Southern Methodist University \\ Dallas, Texas \\ February 17, 1994
}

Host and Chairman: Vic Teplitz, SMU

Organizer: Jack Ritchie, U-Texas Austin

2:00 pm Welcome - Anya Peterson Royce, SMU Vice Provost

Overview of Drell Panel - Jack Ritchie

SSC Assets and Status - Raphael Casper, SSCL

LHC: Report on Fermilab Meeting - Jim Siegrist, SSCL

Contributed Talks:

Peter Rosen, U-Texas Arlington

John Matthews, U-New Mexico

Joe Izen, U-Texas Dallas

Marjorie Corcoran, Rice

Ransom Stephens, U-Texas Arlington

Rainer Meinke, SSCL

Fred 0lness, SMU

Vic Teplitz, SMU

Peter McIntyre, Texas A\&M

Open Discussion 


\section{HEP TOWN MEETING}

\section{University of Chicago \\ Chicago, Illinois}

February 22, 1994

Organizers: Yau Wah, Chicago

Frank Merritt, Chicago

Joel Butler, Fermilab

Contributed Talks:

Support of Ongoing HEP Programs and Extensions

Adrian Melissinos

David Underwood

Maury Goodman

Yau Wah

Mel Shochet

Alvin Tollestrup

Hugh Montgomery

Kaplan (Appel, Christian, Kwan)

LHC Involvement

Dan Green

Brig Williams

Peter Limon

New Hadron Collider Initiatives

W. Foster

Gerald Jackson (D. Herrup, R. Pasquinelli, D. Finley, S. Pruss, P. Lucas, T. Murphy)

Dan Amidei

Jim Freeman

General Issues: Governance, Public Support, Strategic Planning and Considerations

Chip Brock

Heidi Schellman

Bruce Barnett

Ed Hart

Tom Fields

Larry Price

George Gollin, D. Errede, A. El-Khadra, G. Gladding, G. Gollin,

L. Holloway, T. Liss, M. Selen, J. Thaler, and S. Willenbrock

Chip Brock 


\section{HEP Town Meeting}

\section{Lawrence Berkeley Laboratory}

\section{February 28, 1994}

\section{Agenda}

Moderator: R. Cahn, Director Physics Division, LBL

1:00 pm Introductory remarks

Stan Wojcicki (Stanford), chairman of HEPAP

Kevin Einsweiler (LBL), member of HEPAP subpanel

$1: 15 \mathrm{pm}$ Invited presentations

Abe Seiden (UCSC)

Mike Witherell (UCSB)

Barry Barish (Caltech)

2:00 pm Arranged presentations

Steve Olsen (Rochester)

Mike Chanowitz (LBL)

Craig Wuest (LLNL)

Alessandra Ciocio (LBL)

2:30 pm Open discussion

3:00 pm Break

3:15 pm Invited presentations

Uriel Nauenberg (Colorado)

Dave Ritson (SLAC)

Marjorie Shapiro (LBL)

4:00 pm Arranged presentations

Katsushi Arisaka (KEK)

Gil Gilchriese (LBL)

Geoff West (LASL)

Ann Heinson (UC Riverside)

4:30 pm Open discussion 


\section{HEP TOWN MEETING \\ Harvard University \\ Cambridge, Massachusetts}

March 23, 1994

Organizer: John Huth, Harvard

Contributed Talks:

J. Huth

C. Baltay

A. J. Smith

Accelerators
A. Bodek
Palmer
O'Rear
Roser

Remarks on LHC

Golden

Lane

Bensinger

Roberts

D. Cutts

Kaons, Neutrino's, B's and QCD

Solowar

L. Littenburg

Murtagh

Jaffee

Willutski

Marx

Cox

Tanenbaum

Winn

Min Chen

U. Becker

Wlodek Guryn 
Town Meetings on Particle Astrophysics and Non-Accelerator Physics

Tuesday, February 8

3:00 p.m. - 6:00 p.m.

University of Pennsylvania, Philadelphia

Moderator: E. Beier

Approximately 30 participants

Wednesday, February 16

10:00 a.m. - 2:00 p.m.

University of California at Los Angeles

Moderator: B. Barish

Approximately 60 participants

Saturday, March 12

2:30 p.m. - 5:00 p.m.

Fermi National Laboratory

Moderator: E. Kolb

Approximately 60 participants

Monday, March 14

12:00 p.m. - 2:00 p.m.

Harvard University

Moderator: R. Lanou

Approximately 10 participants

These meetings all had the same format. After a short summary of the purpose of the HEPAP subpanel and perceived issues by $B$. Sadoulet, a lively discussion took place, often with a few transparencies from members of the audience. 
Appendix G

The subpanel received many thoughtful letters from our colleagues. These letters were extremely valuable to us in our work. We wish to acknowledge the approximately 400 scientists, whose names are listed in this appendix, for sharing with us their ideas, recommendations, concerns, and aspirations for the future of particle physics. We also acknowledge receiving two important petitions with a total of about 1100 signatures. 
ABASHIAN Alexander (Virginia Tech.)

ADAM Ian (Columbia U.)

ALBROW Michael (Fermilab)

ALEKSAN R. (Saclay, France)

AMADEI Dan (U. Michigan)

ANDERSON Gregory (MIT)

ANSELMI R.T.

APPEL Jeffrey (Fermilab)

ARNOWITT Richard (Texas A\& M)

ARYAL Mukunda (Kansas State)

AYRES David (Argonne)

BAIRD Ken (Rutgers NY)

BALAMURALI V. (U. of Notre Dame)

BALTAY Charles (SLAC)

BARLETTA William (LBL)

BARNETT Bruce (Johns Hopkins)

BARS Itzhak (USC)

BARTELT John (Vanderbilt U.)

BARTON Joe, Congressman (WDC)

BAUER Ulrich (Florida State)

BENSINGER James (Brandeis U.)

BERKELMAN Karl (Cornell)

BERNSTEIN Robert (Fermilab)

BERTRAM Iain A. (Rice U.

BHATNAGAR Vipin (Panjab U.)

BJORKEN James (SLAC)

BLACKETT Gavin (U. Tennessee)

BLACKETT Kathleen (U. Tennessee)

BLAIR Bob (Argonne)

BLUMENFELD Henry (France)

BODEK Arie (U. Rochester) 
BOEHM Felix (Calif. Inst. of Technology)

BOLOGNESE T. (Saclay, France)

BORDERS John D. (U. of Rochester)

BOSTED Peter (SLAC)

BOWER Gary (SLAC)

BOYA Luis (Spain)

BRANDENBURG George (Harvard)

BRAU Jim (U. Oregon)

BREAKSTONE Alan (U. Hawaii)

BREIDENBACH Martin (SLAC)

BRIERE Roy (U. Chicago)

BROCK Raymond (Michigan State U.)

BRODSKY Stanley (SLAC)

BUGG William (U. Tennessee)

BURROWS Philip (MIT)

CAHILL Kevin (U. New Mexico)

CAHN Robert (LBL)

CALDWELL David (UC Santa Barbara)

CARITHERS Bill (Fermilab)

CARLSMITH Duncan (U. of Wisconsin)

CARRIGAN Richard (Fermilab)

CASEY Dylan (Fermilab)

CHANOWITZ Michael (LBL)

CHEN Pisin (SLAC)

CHIVUKULA Sekhar (U. Boston)

CHRISTIAN David C. (Fermilab)

CHURCH Eric (U. Washington)

CLINE David (UCLA)

COHEN Andrew (Boston U.)

CONDO George (U. Tennessee)

CONRAD Janet (Fermilab) 
COOK Victor (U. Washington)

COX Brad (U. Virginia)

CRAWFORD Glen (Cornell)

CROWELL Lawrence B. (U. New Mexico)

CRUETZ Michael (Brookhaven)

CSORNA Steve (Vanderbilt)

CUMMINGS M.A.C. (U. Hawaii)

DAMERELL Chris (Rutherford Appleton Lab., England)

DAVIS III Austin

DEMARTEAU Marcel (Fermilab)

DERRICK M. (Argonne)

DINE Michael (SLAC)

DORFAN Jonathan (SLAC)

DRAPER Paul (U. Texas)

DRELL Persis (Cornell)

DUBOSCQ J.E. (Switzerland)

DUGAN Michael (Boston U.)

DZIERBA Alex (U. Indiana)

EDELSTEIN Richard (Carnegie Mellon)

EINHORN Marty (U. Michigan)

ENGLER Arnold (Carnegie Mellon)

FABJAN Christian W. (CERN)

FALK Adam (Johns Hopkins)

FARRAR Glennys (Rutgers NY)

FATYGA M. Kathryn (U. of Rochester)

FATYGA Mirek (Brookhaven)

FELDMAN Gary (Harvard)

FERBEL Tom (U. Oregon)

FERGUSON Thomas A. (Carnegie Mellon)

FIELDS Tom (Argonne)

FISHER Peter (Johns Hopkins) 
FITCH Val (Princeton)

FLATTUM Eric (Michigan State U.)

FRAME Katherine C. (Michigan State U.)

FRANKLIN Melissa (Harvard)

FREY Raymond (U. Oregon)

FRIED H.M. (Brown U.)

FRISCH Henry (U. Chicago)

FRY J.R. (U. of Liverpool)

FUESS Theresa (Fermilab)

GABRIEL Tony (Oak Ridge, Tennessee)

GAIDOT A. (Saclay, France)

GAILLARD Mary (UC Berkeley)

GALIK Richard (Cornell)

GARBINCIUS Peter (Fermilab)

GELD Terece L. (Michigan State U.)

GELFAND Norman (Fermilab)

GENIK II R.J. (Michigan State U.)

GENSLER Steve (U. Chicago)

GEORGE Jean (U. New Mexico)

GEORGI Howard (Harvard)

GERDES David (U. Michigan)

GILCHRIESE Murdock (LBL)

GILMAN Fred (SSC)

GIORGI, Marcello (U. of Pisa and INFN Pisa)

GLASHOW Shelley (Harvard)

GLENN Steven M. (UC, Davis)

GOLDEN Mitchell (Harvard)

GOLLIN George (U. Illinois)

GOLDSTONE Jeffrey (MIT)

GOODMAN Jordan (U. Maryland)

GORDON Howard (Brookhaven) 
GOSHAW Al (Duke U.)

GOSS Lewis Taylor (Texas A\& M)

GOULIANOS Dino (U. Rockefeller)

GRAHAM Greg (U. Chicago)

GRANNIS Paul (SUNY Stony Brook)

GRIFFIN Gregory L. (UC, Irvine)

GUGLIELMO Gerald (U. Minnesota)

GUNION J.F. (UC Davis)

HABER Howard (SLAC)

HAHN Ki Suk (U. of Rochester)

HALL Raymond E. (UC, Riverside)

HANDLER Thomas (U. Tennessee)

HANSON Gail (U. Indiana)

HARRIS Deborah (U. Chicago)

HART Edward (U. Tennessee)

HARTILL Don (Cornell)

HARTMAN Keith

HAUSER Jay (UCLA)

HEINTZ Ulrich (Columbia U.)

HERTZBACH Stan (U. Massachusetts)

HEUSCH Clemens (UC Santa Cruz)

HEYLING G.H. (U. New Mexico)

HILL Chris (Fermilab)

HINCHLIFFE Ian (LBL)

HIROSKY R. (Florida State U.)

HITLIN David (CalTech)

HOHLMANN Marcus (U. Chicago)

HOJVAT Carlos (Fermilab)

HOLMAN Richard F. (Carnegie Mellon)

HOLMES Stephen (Fermilab)

HOUK Gary (Fermilab) 
HUGHES Vernon (Yale)

HULL McAllister (U. New Mexico)

IMLAY R. (Louisiana State U.)

INNES Walt (SLAC)

ITO Mark (Princeton)

JARLSKOG Goran (Sweden)

JARRY P. (Saclay, France)

JERGER Steven A. (Michigan State U.

JOFFE-MINOR Tacy (Northwestern U.)

JOHARI Hossien (Northwestern U.)

JOHNSON Kenneth (MIT)

JONES Lawrence (U. Michigan)

JONES Michael (U. Hawaii)

JOVANOVIC Drasko (Fermilab)

KAJIKAWA Ryoichi (Nagoya U.)

KAMON Teruki (Texas A\& M)

KANE Gordy (U. Michigan)

KAPLAN Daniel (Fermilab)

KARCHIN Paul (Yale)

KELLER Stephene (Florida State)

KEMMELL Bruce M. (U. New Mexico)

KENDALL Henry (MIT)

KHAN Irshadullah (Bentley College)

KIM Yongduk (Sogang U.)

KIMURA Wayne (STI Optronics)

KIRK Thomas B. (SSC)

KOTCHER Jonathan (Brookhaven)

KRAEMER Robert W. (Carnegie Mellon)

KRAYBILL James Colby (U. New Mexico)

KWAN Simon W.L. (Fermilab)

KYCIA Thaddeus (Brookhaven) 
LACH Joseph (Fermilab)

LAI Kwan-Wu (U. Arizona)

LANDSBERG Greg (SUNY Stonybrook)

LANE Kenneth (U. Boston)

LANG Karol (U. Texas)

LANGACKER Paul (U. Pennsylvania)

LANKFORD Andrew (UC Irvine)

LANOU Bob (Brown U.)

LAYTER John (UC Riverside)

LEDERMAN Leon (Fermilab)

LEMAIRE M.C. (Saclay, France)

LENNOX Arlene (Fermilab)

LESQUEN A. de (Saclay, France)

LEVINE Michael J. (Carnegie Mellon)

LI Ling-Fong (Carnegie Mellon)

LIMON Peter (Fermilab)

LING James (JPL)

LING T.Y. (Ohio State U.)

LIPKIN Harry (Weizmann)

LIPTON Ronald (Fermilab)

LISS Tony (U. Illinois)

LITT Larry (UCSF)

LITTENBERG Laurence (Brookhaven)

LONDON G. (Saclay, France)

LOPEZ Jorge (Texas A\& M)

LOSECCO J.M. (U. of Notre Dame)

LOW Francis (MIT)

LOWENSTEIN Derek (Brookhaven)

LUBATTI Henry (U. Washington)

LUTZ A.M. (France)

LYNCH Harvey (SLAC) 
MACCALLUM Crawford (U. New Mexico)

MALLIK U. (Iowa U.)

MANLY Steven (Yale)

MANN Alfred K. (U. Pennsylvania)

MANTOVANI Giancarlo (Universita di Perugia)

MARCH Robert (U. of Wisconsin)

MATTHEWS John (U. New Mexico)

MAULDIN Jim (Colorado)

MCDANIEL Boyce (Cornell)

MCFARLANE David (McGill U.)

MCINTYRE Peter (Texas A\& M)

MCKENNA Janis (U. British Columbia)

MCKIBBEN Thomas (U. of Illinois, Chicago)

MELANSON Harry (Fermilab)

MELISSINOS A.C. (U. Rochester)

MILLER David (Purdue)

MIYASHITA Shige (U. Usukuba, Japan)

MO L. (VA Polytechnic Inst. and State U.)

MONIZ Ernie (MIT)

MONTCHENAULT G. Hamel de (Saclay, France)

MONTGOMERY Hugh (Fermilab)

MORRISON Rollin (UC Santa Barbara)

MORSE William (Brookhaven)

NANG Freedy (Brown U.)

NANOPOULOS Dimitri (Texas A\& M)

NARAIN M. (Fermilab)

NAUENBERG Uriel (U. Colorado)

NEIS Eric (U. Michigan)

NGUYEN Ai G. (Kansas State)

NODULMAN Larry (Argonne)

NORRIS James (Kansas State) 
OGREN Harold (U. Indiana)

OREAR Jay (Cornell)

OREGLIA Mark (U. Chicago)

OZAKI Satoshi (Brookhaven)

PAIGE Frank (Brookhaven)

PANVINI Bob (U. Vanderbilt)

PARTRIDGE Richard (Brown)

PELLEGRINI Claudio (UCLA)

PEOPLES John (Fermilab)

PERUZZI Ida (Lab. Nazionali di Frascati)

PESKIN Michael (SLAC)

PIEMONTESE Livio (INFN Sezione di Ferrara)

PIERRE F. (Saclay, France)

PIILONEN Leo (Virginia Tech.)

PILCHER Jim (U. Chicago)

PITMAN Dale (U. Victoria)

PLANO Richard (Rutgers NY)

PLUNKETT Robert (Fermilab)

POIRIER John (U. Notre Dame)

PONDROM Lee (U. of Wisconsin)

PORDES Stephen (Fermilab)

PORTER Frank (Calif. Inst. of Technology)

PREPOST Richard (U. Wisconsin)

PRESCOTT Charles (SLAC)

PRICE Lawrence (Argonne)

PRICE Richard Marcus (U. New Mexico)

PRIMACK Joel (UC Santa Cruz)

PROCARIO Michael (Carnegie Mellon)

PROTOPOPESCU Serban (Brookhaven)

PUNKAR Greg (SLAC)

QUIGG Chris (Fermilab) 
QUIGLEY James (MIT)

QUINTAS Paul Z. (Fermilab)

RADEKA Veljko (Brookhaven)

RAGHAVAN Raju (AT\& T Bell Lab.)

RAMBERG Erik (Fermilab)

RAMOND Pierre (U. Florida)

RANDALL Lisa (MIT)

RATCLIFF Blair (SLAC)

RAU Ronnie (DESY)

REAY Neville W. (Kansas State)

REEDER Don (U. of Wisconsin)

REINES F. (UC Irvine)

RICHTER Burton (SLAC)

ROE Byron (U. Michigan)

ROE Natalie (LBL)

ROMANO Jim (U. Chicago)

ROSNER Jonathan (U. Chicago)

ROWSON Peter (Columbia U.)

RUCHTI Randy (U. Notre Dame)

RUIZ Debra D. (U. New Mexico)

RUSS James S. (Carnegie Mellon)

RUTHERFORD John (U. Arizona)

SADROZINSKI Hartmut F.W. (UC Santa Cruz)

SALTZBERG David (U. Chicago)

SANDWEISS Jack (Yale)

SCHALK Terry (UC Santa Cruz)

SCHINDLER Rafe (SLAC)

SCHMIDT Michael (Yale)

SCHUBERT Klaus (Technische Universitaet Dresden)

SCHUNE M.H. (France)

SCHWARTZ Alan (Princeton) 
SCHWINGENHEUER Bernard (U. Chicago)

SCIULLI F. (Columbia U.)

SEIDEN Abraham (UC Santa Cruz)

SHAEVITZ Michael (Columbia U.)

SHAPIRO GIL (LBL)

SHAWHAN Peter (U. Chicago)

SHOCHET Melvyn (U. Chicago)

SHROCK Robert (SUNY Stony Brook)

SIDWELL Ronald A. (Kansas State)

SIEGRIST James (UC Berkeley and SSC)

SIEMANN Robert (SLAC)

SIMMONS Elizabeth (Boston U.)

SKUJA Andris (U. Maryland)

SLIWA Krzysztof (Tufts U.)

SMITH Stew (Princeton)

SMITH Wesley (U. of Wisconsin)

SNOW George (U. Maryland)

SOLOMEY Nickolas (U. Chicago)

SONI Amarjit (Brookhaven)

SPENCER James (SLAC)

STANTON Noel R. (Kansas State)

STEINHARDT Paul (U. Pennsylvania)

STEPHENS Ransom (U. Texas)

STICKLAND David (CERN)

STONE Sheldon (U. Syracuse)

STRAUSS Michael (SLAC)

STREETS Kathleen (Fermilab)

STROVINK Mark (LBL)

SUTTON Roger B. (Carnegie Mellon)

TANNENBAUM M.J. (Brookhaven)

TAYLOR Cyrus (Case Western Reserve U., Cleveland) 
TAYLOR Frank (MIT)

TAYLOR Richard (SLAC)

TAYLOR Tracy (Northwestern U.)

TENEMBAUM Peter (U.C. Santa Cruz)

TEPLITZ Vigdor (Southern Methodist U., Dallas)

THORNDIKE Alan (U. Puget Sound)

THORNDIKE Ed (U. Oregon)

THUN Rudolph (U. Michigan)

TIMKO Mark (U. Tufts)

TIMM Steven (Carnegie Mellon)

TOBACK David (U. Chicago)

TOKI Walter (Colorado State)

TOLLESTRUP Alvin (Fermilab)

TREIMAN Sam (Princeton)

TRILLING George (LBL)

TRIPATHI Arun K. (Kansas State)

TRUEMAN Larry (Brookhaven)

TUMER Tumay (UC Riverside)

TURLUER M.L. (Saclay, France)

VASSEUR G. (Saclay, France)

VOGEL Helmut (Carnegie Mellon)

WALI K.C. (Syracuse U.)

WANG Jinsong (U. Chicago)

WARD Bennie (U. of Tennessee, Knoxville)

WATTS Steve (Brunel U.)

WEBB Robert (Texas A\& M)

WEINSTEIN Alan (Calif. Inst. of Technology)

WEINSTEIN Roy (U. Houston)

WELCH James (Cornell)

WENDLING Mike (lowa State U.)

WHITE James (Texas A\& M) 
WHITMORE J. (DESY)

WHITMORE James J. (Pennsylvania State U.)

WILLIAMS Hugh (U. Pennsylvania)

WILLIS William (U. Columbia)

WILSON Bob (Colorado State)

WILSON Richard (Harvard)

WINSTEIN Bruce (U. Chicago)

WIRJAWAN Johannes (Texas A\& M)

WITCHEY Nicholas J. (Kansas State)

WITHERELL Michael (UC Santa Barbara)

WOJCICKI Stan (SLAC)

WOLBERS Stephen (Fermilab)

WOLFE David (U. New Mexico)

WOLFENSTEIN Lincoln (Carnegie Mellon)

WORMSER G. (France)

WRIGHT Douglas (LLNL)

WU Sau Lan (U. Wisconsin)

WUEST Craig (LLNL)

WUGUANG Yan (Inst. of HEP Beijing)

XU Chibing (U. New Mexico)

YAMAMOTO Richard (MIT)

YANG Shih-Wen (Kansas State)

YODH Gaurang (UC Irvine)

YOKOSAWA A. (Brookhaven)

YUTA Haruo (Tohoko U.)

ZAPALAC Geordie (SLAC)

ZHANG Chong (Kansas State)

ZHU Zhonghui (U. of Rochester)

ZIMMERMAN David (CERN)

ZITO M. (Saclay, France) 


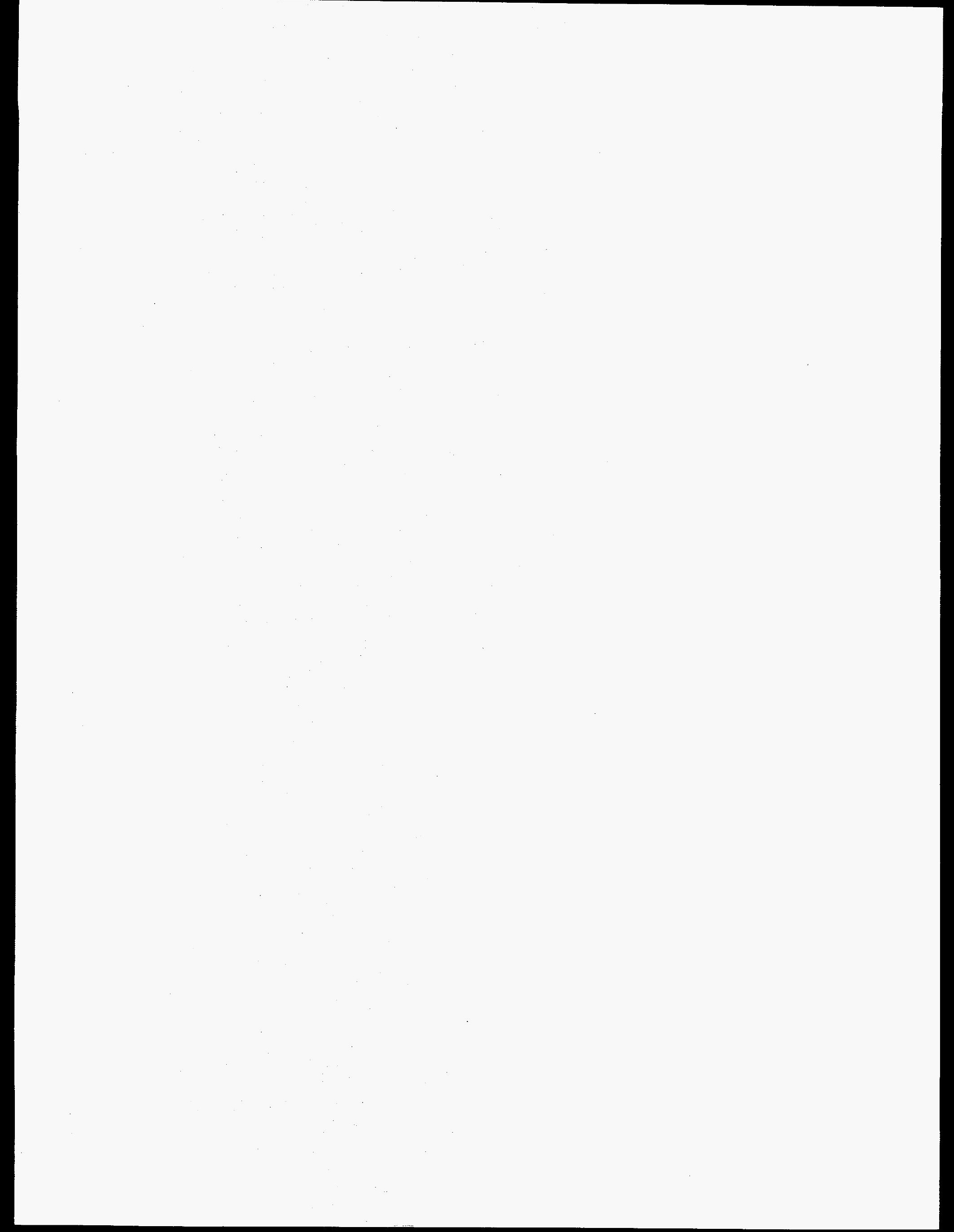


Appendix $\mathrm{H}$ 


\section{Guidel ines proposed by ICFA for the Interregional}

\section{Utilization of Major Regional Experimental Facilities}

\section{for High-Energy Particles Physics Research}

(Agreed by ICFA at its Fifth Meeting held at CERN on 9 July 1980)

- Considering that in the future major experimental facilities for high energy particle physics research, notably the very largest particle accelerators and colliding beam machines, are likely to be few in number, probably only one of each type of the very highest energy and that these machines will be located in different regions of the world,

- And recognizing that experimental physicists from all regions will wish to gain access to these few machines in order to pursue their research,

- ICFA proposes that the regional laboratories operating these facilities should adopt a common policy towards experimental physicists from other regions seeking to use the facilities they operate. The guidelines proposed are as follows:

1. The selection of experiments and the priority accorded to them are the responsibility of the Laboratory operating the regional facility.

2. The criteria used in selecting experiments and determining their priority are:
(a) scientific merit
(b) technical feasibility
(c) capability of the experimental group
(d) availability of the resources required.

3. It is expected that teams from other regions will normaliy wish to join with local regional teams to form experimental groups in proposing and carrying out experiments using a regional facility. The national or institutional affiliations of the teams should not influence the selection of an experiment nor the priority accorded to it.

4. The availability of the resources needed for the experiment are examined at the time of selection of the experiment (sec. 2 (d) above). The contributions of each team and of the Operating Laboratory to an experiment are the subject of agreements drawn up between the Operating Laboratory and the authorized leaders of the teams in the experimental group. When appropriate, realization of the proposals approved may be effected within the framework of bilateral and multilateral agreements in force or newly reached arrangements.

5. Operating Laboratories should not require experimental groups to contribute to the running costs of the accelerators or colliding beam machines nor to the operating costs of their associated experimental areas. 
6. It is expected that averaged over a reasonable period of time the application of guideline 2. above will lead to a balanced use of the major new facilities by the regions concerned. However, if at any time an Operating Laboratory finds that the participation of teams from other regions in their experimental program is becoming excessive, the Operating Laboratory may be obliged to limit that participation. Any such action should be accompanied by discussions with the relevant authorities of the regions concerned and consultations with the other Operating Laboratories subscribing to the Guidelines laid down in this document. 